


\section{Fast and non-invasive Raman imaging of material distributions at interfaces}

An algorithm-improved confocal Raman microscopy study

Sachin Shivaji Nair 


\section{Graduation committee:}

$\begin{array}{ll}\text { Prof. dr. J.L. Herek } & \text { University of Twente, Chairperson } \\ \text { Prof. dr. F.G. Mugele } & \text { University of Twente, Promotor } \\ \text { Dr. C. Otto } & \text { University of Twente, Assistant promotor } \\ \text { Dr. M.H.G. Duits } & \text { University of Twente, Assistant promotor }\end{array}$

\section{Members:}

Prof. dr. ir. H.J.W. Zandvliet University of Twente

Prof. dr. G. Mul

University of Twente

Prof. dr. W.R. Browne

University of Groningen

Dr. F. Ariese

Vrije Universiteit Amsterdam

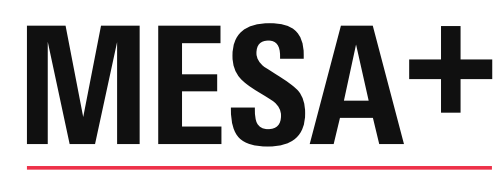

INSTITUTE FOR NANOTECHNOLOGY
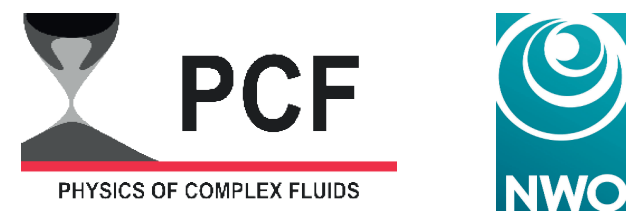

The research described in this thesis was performed at the Physics of Complex Fluids group within the MESA+ Institute for Nanotechnology and the Department of Science and Technology of the University of Twente. This work is part of the research program Rock-on-a-Chip, which is partly financed by the Netherlands Organisation for Scientific Research (NWO) and through the Exploratory Research (ExploRe) program of BP plc.

Title: Fast and non-invasive Raman imaging of material distributions at interfaces Author: Sachin S. Nair

ISBN: 978-90-365-5312-4

DOI: $10.3990 / 1.9789036553124$

Copyright (C) 2021 Sachin Shivaji Nair, The Netherlands.

All rights reserved. No parts of this thesis may be reproduced, stored in a retrieval system or transmitted in any form or by any means without permission of the author. Alle rechten voorbehouden. Niets uit deze uitgave mag worden vermenigvuldigd, in enige vorm of op enige wijze, zonder voorafgaande schriftelijke toestemming van de auteur. 
Cover design by Sachin Nair.

Printed by Gildeprint, Enschede. 


\section{FAST AND NON-INVASIVE RAMAN IMAGING OF MATERIAL DISTRIBUTIONS AT INTERFACES}

\section{DISSERTATION}

to obtain

the degree of doctor at the Universiteit Twente, on the authority of the rector magnificus, prof. dr. ir. A. Veldkamp, on account of the decision of the Doctorate Board, to be publicly defended on Thursday 16 December 2021 at 16.45 hours

by

Sachin Shivaji Nair

born on the 6th of February, 1993

in Mahayel, Saudi Arabia 
This dissertation has been approved by:

Promotor:

Assistant promotor:

Assistant promotor:
Prof. dr. F.G. Mugele

Dr. C. Otto

Dr. M.H.G. Duits 



\section{Contents}

1 Introduction 1

1.1 Raman scattering . . . . . . . . . . . . . . . 1

1.2 Raman imaging - limitations .............. . . 4

1.3 Motivation - imaging in the low SNR regime . . . . . . . . 7

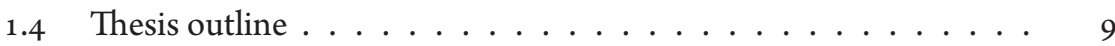

2 ai-CRM: principle and technique 15

2.1 Confocal Raman microscopy $(\mathrm{CRM}) \ldots \ldots . \ldots . . \ldots 16$

2.2 Algorithm-improved CRM (ai-CRM) . . . . . . . . . . . . 19

2.2.1 Singular Value Decomposition .......... 20

2.2.2 Principal Component Analysis .......... . 25

2.2.3 PCA-based denoising of Raman HSD . . . . . . . . . 27

3 Improved Raman imaging of $2 \mathrm{D}$ materials 33

3.1 Introduction $\ldots \ldots \ldots . \ldots \ldots \ldots$

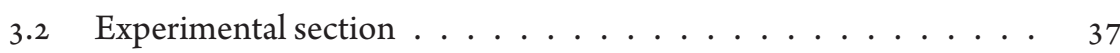

3.3 Results and Discussion ................ 38

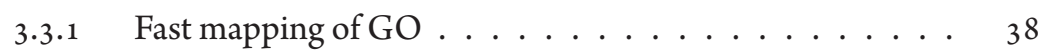

3.3 .2 Non-invasive mapping of GO . . . . . . . . . 43

3.3.3 Fast mapping/quality analysis of graphene ... . . . . 47

3.3 .4 Fast mapping of other $2 \mathrm{D}$ materials ... . . . . . 49

3.3.5 Fast volumetric Raman imaging . . . . . . . . . . 49

3.3.6 Fast mapping of $2 \mathrm{D}$ materials on arbitrary substrates . . 53

3.4 Conclusion ...................... 55 
4 Improved analyte detection using ai-GERS

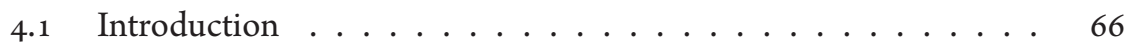

4.2 Experimental section ................ 69

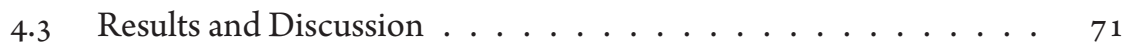

4.3.1 rGO as a GERS substrate ............ 71

4.3.2 Limit of detection on $\mathrm{rGO} \ldots \ldots . \ldots 75$

4.3.3 Limit of detection on oxidized graphene . . . . . . 78

4.3.4 Applications of $i n-s i t u$ ai-GERS . . . . . . . . . . 83

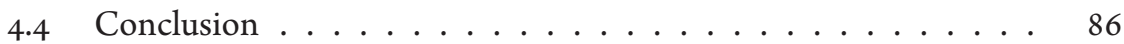

5 Improved 3D-Raman imaging for EOR studies 93

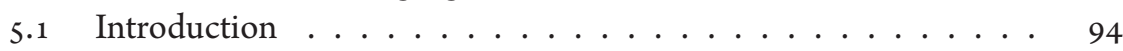

5.2 Experimental section . . . . . . . . . . . . . . . 98

5.3 Results and Discussion ................ 101

5.3.1 Reactive wettability alteration on mica . . . . . . 103

5.3.2 Reactive wettability alteration on silica . . . . . . . . 109

5.3.3 Surfactant redistribution post wettability alteration . . . . 111

5.3.4 Substrate-dependent contact angle change ...... 116

5.3.5 Wettability alteration mechanism ......... 118

5.4 Conclusion .................... 119

$\begin{array}{lr}\text { Summary } & 127\end{array}$

$\begin{array}{lr}\text { Samenvatting } & 131\end{array}$

$\begin{array}{lr}\text { List of publications } & 135\end{array}$

$\begin{array}{lr}\text { Acknowledgments } & 137\end{array}$

$\begin{array}{ll}\text { About the author } & 141\end{array}$ 
"Look at the resplendent colours on the soap bubbles!

Why is the sea blue?

What makes diamond glitter?

Ask the right questions, and nature will open the doors to her secrets."

Sir C.V. Raman

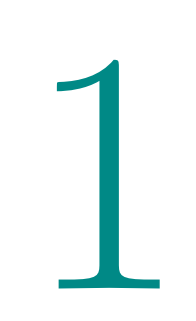

Introduction

IN THIS CHAPTER I will introduce the main concepts relevant to understanding the background and motivation of this research. Towards the end, the chapter-wise layout of this thesis is elucidated.

\subsection{Raman scattering}

Spectroscopy is the field of science that deals with the study of light-matter interactions. Analysing the results of spectroscopic measurements allows for probing the physical and chemical properties of materials. Typically, when an electromagnetic 


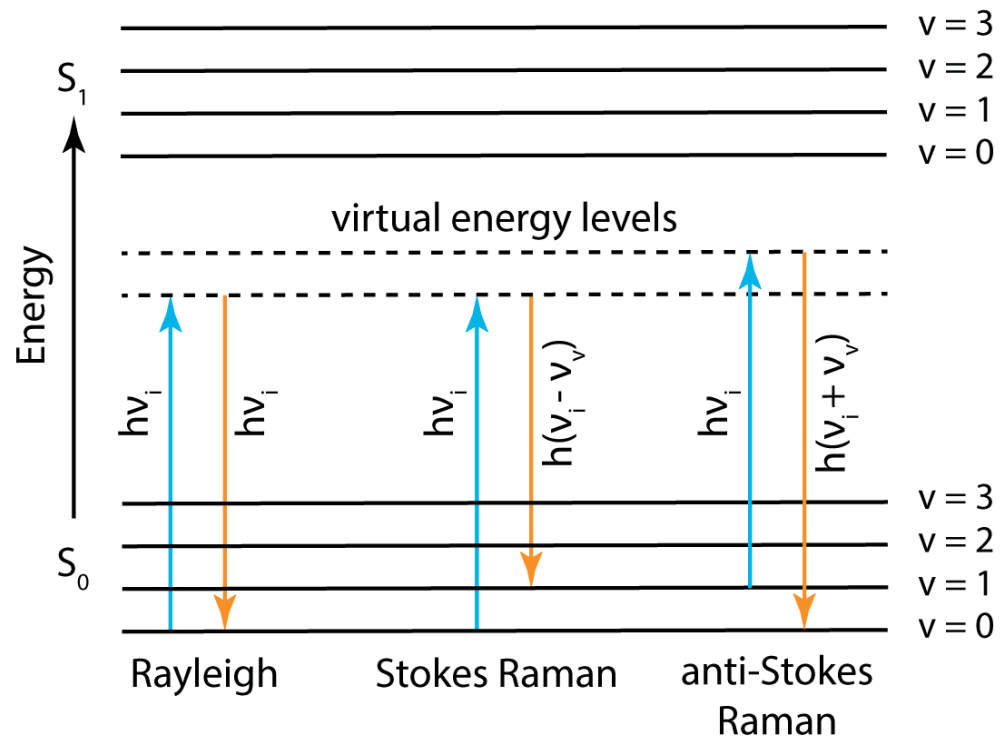

Figure 1.1: Energy level diagram and transitions from the ground state to a virtual excited state involved in Rayleigh, stokes Raman and anti-Stokes Raman scattering. $S_{i}$ refers to the $i^{\text {th }}$ electronic level whereas $v=i$ refers to the $i^{\text {th }}$ vibrational level of the molecule. The energy required for excitation from the ground state to the virtual energy level is $h v_{i}$, and to the first vibrational level is $h v_{v}$, respectively.

wave interacts with matter, the constituting particles (subatomic, atomic, molecular) can absorb, emit and/or scatter radiation. Scattering, unlike absorption/emission, does not require the energy of the photon to match with the particle's transition energy between two quantized levels. Light scattering by particles much smaller than the wavelength of light is a consequence of their electric polarizability. In response to light's oscillating electric field, the charge distribution in the particle also starts to oscillate, thereby emitting radiation which we see as scattered light. In terms of energy diagrams, scattering is depicted as the absorption and spontaneous re-emission of a photon (2-photon process) after excitation of the particle to a virtual energy state (Figure 1.1). 
On the basis of the net energy transferred between the photon and the scatterer, two types of scattering are observed. A majority of the photons are scattered elastically, implying that there is no difference in the energy of the scattered photon relative to the incident one. This is known as Rayleigh scattering. On the other hand, a small fraction of the photons are scattered inelastically, such that the net energy transferred to the scatterer is non-zero and equal to the energy required to excite the molecule to a higher vibrational level (Figure 1.1). This is known as Raman scattering or the Raman effect ${ }^{[1]}$, named after the Indian physicist Sir C.V. Raman who discovered the phenomenon in 1928 along with his student K.S. Krishnan. Depending on whether the vibrational energy of the molecule is added to or removed from the incident photon energy, Raman scattering can be further classified into Stokes and anti-Stokes Raman scattering, respectively. For the sake of brevity in this thesis, Raman scattering henceforth refers to only Stokes Raman scattering, i.e., when there is a decrease in frequency of the outgoing photon with respect to the incoming one due to the aforementioned energy transfer.

By now, it is clear that the inelastic nature of Raman scattering can be exploited to access the vibrational modes of a molecule. However, not every vibrational mode can be accessed, or in other words, not every vibrational mode is Raman-active. The basic selection rule pertaining to Raman scattering necessitates a modulation in polarizability of the molecule with the corresponding vibrational coordinate. This is unlike infrared (IR) absorption spectroscopy, which entails a vibrational motion to change the dipole moment of the molecule in order to be IR-active. The change in energy of the Raman-scattered photon relative to the excitation energy can be used as a fingerprint to identify distinct molecular vibrations in Raman-active materials. This energy difference is commonly represented by a relative shift in wavenumbers, also called as the Raman shift, as shown below.

$$
\text { Raman shift }\left(\mathrm{cm}^{-1}\right)=\Delta \bar{\omega}=\frac{10^{7}}{\lambda_{\text {excitation }}[\mathrm{nm}]}-\frac{10^{7}}{\lambda_{\text {scattered }}[\mathrm{nm}]}
$$


For example, consider a molecule that is excited at a wavelength, $\lambda_{\text {excitation }}=532 \mathrm{~nm}$, or an absolute wavenumber of $18797 \mathrm{~cm}^{-1}$. According to equation 1.1, a Raman band appearing at shift of $1000 \mathrm{~cm}^{-1}$ would imply that the radiation is scattered and red-shifted to a wavelength of $\approx 562 \mathrm{~nm}$. To access the same vibrational mode using absorption spectroscopy, the molecule has to absorb at an IR wavelength of $10 \mu \mathrm{m}$. This difference highlights the importance of Raman spectroscopy as an analytical technique - molecular vibrations can be probed using visible monochromatic radiation and the relative shift in wavenumbers does not depend on the wavelength of excitation used.

A typical Raman spectrum is obtained by plotting the intensity of the scattered radiation as a function of the aforementioned Raman shift. The intensity can be represented by the total number of Raman scattered photons $\left(n_{R}\right)$ generated from a probed volume of molecules $(V)$. In simple terms, $n_{R}$ is directly proportional to the number of scattering molecules $(N)$ present in the probed volume, the probed length $(z)$, the Raman scattering cross-section $(\mathrm{d} \sigma / \mathrm{d} \Omega)$, the scattering solid angle $(\mathrm{d} \Omega)$ and the number of incident photons used for excitation $\left(\frac{P t}{\hbar v}\right)$, as shown below:

$$
n_{R} \approx\left(\frac{N}{V}\right) z\left(\frac{\mathrm{d} \sigma}{\mathrm{d} \Omega}\right) \mathrm{d} \Omega\left(\frac{\mathrm{Pt}}{\hbar v}\right)
$$

where $\mathrm{P}$ and $\mathrm{t}$ are the power and exposure time of the laser used, respectively.

\subsection{Raman imaging - limitations}

In addition to being a very important analytical technique for chemical identification, the combination of an optical microscope with a Raman system can also be used for performing Raman imaging. The feasibility of Raman micro-spectroscopy to locally probe the chemical properties of materials in a label-free manner and with sub-micron spatial resolution has been exploited in a multitude of fields ${ }^{[2]}$, like earth 
sciences $^{[3]}$, pharmaceuticals ${ }^{[4]}$, biological cell imaging ${ }^{[5]}$ and material sciences ${ }^{[6]}$ to name a few.

A Raman image can be created by locally monitoring the properties of the Raman bands (like band area, amplitude, peak position, full width at half maximum etc.) of interest as a function of the spatial coordinates over which a large number of spectra have been acquired. This mode of Raman imaging is known as point-by-point mapping, and is most commonly used, although other modes like line scanning [7] and global imaging ${ }^{[8]}$ exist. A typical Raman map is made by pre-specifying the image resolution (pixels/distance), the probing laser power $(\mathrm{mW})$ and the spectral exposure or accumulation time (seconds/pixel). The standard Raman scattering cross-section for non-resonant excitation $(\mathrm{d} \sigma / \mathrm{d} \Omega)$ is of the order of $\approx 10^{-34} \mathrm{~m}^{2} / \mathrm{sr}$. Despite this low intensity of Raman scattered photons, with the advent of better laser sources, Rayleigh line rejection filters and charge-coupled device (CCD)-based sensitive detectors, modern day confocal Raman microscopes (CRM) can provide a good signal-to-noise ratio (SNR) of a Raman spectrum that is acquired using a laser power of several tens of milliwatts (focused over a few $\mu \mathrm{m}^{2}$ area of the laser spot) and an exposure time of a few seconds/pixel.

This, however, is still not feasible for performing $2 \mathrm{D} / 3 \mathrm{D}$ Raman imaging of materials at a decent (diffraction-limited) spatial resolution. To understand why, let us estimate the number of Raman scattered photons $\left(n_{R}\right.$, equation 1.2$)$ generated from probing a $100 \mathrm{~nm}(z)$ thin, densely packed multilayer of deuterated-stearic acid (d-SA, $\left.\mathrm{C}_{17} \mathrm{D}_{35} \mathrm{CO}_{2} \mathrm{H}\right)$, similar to the system studied in Chapter 5 of this thesis. The height of a vertically-aligned molecule of stearic acid is $\approx 2.5 \mathrm{~nm}$, with a cross-sectional area of roughly $0.2 \mathrm{~nm}^{2} /$ molecule ${ }^{[9,10]}$. Thus, within a cylindrical probed volume $(V)$ of $\approx 0.05 \mu \mathrm{m}^{3}$, the total number of molecules $(N)$ present is $1 \times 10^{8}$. The Raman scattering cross-section $(\mathrm{d} \sigma / \mathrm{d} \Omega)$ of any $\mathrm{d}$-SA vibration is assumed to be $\approx 10^{-34}$ $\mathrm{m}^{2} / \mathrm{sr}^{[11]}$ and the collecting solid angle, $\mathrm{d} \Omega \approx \pi \mathrm{sr}$. For an excitation wavelength of $\lambda=532 \mathrm{~nm}$ and a typical probing laser power of $10 \mathrm{~mW}$, equation 1.2 gives: 


$$
\begin{gathered}
n_{R} \approx\left(\frac{1 \times 10^{8}}{5 \times 10^{-20}}\right) \times 10^{-7} \times\left(10^{-34}\right) \times \pi \times\left(\frac{10^{-2} \times 1}{6.63 \times 10^{-34} \times 5.64 \times 10^{14}}\right) \\
n_{R} \approx 1700 \text { Raman photons/second }
\end{gathered}
$$

Note that this value is only an estimate and in reality the actual number of detected photons will be lower, depending on instrumental factors like the quantum efficiency (QE) of the detector, collection efficiency of the optics, pinhole diameter etc. Nevertheless, the SNR is sufficient (typical noise levels in CCD-based Raman systems are much lower than this estimated value of the signal, $n_{R}{ }^{[12]}$ ) to get reliable information from the d-SA layer at the probed pixel. Conventionally, it is not uncommon for researchers to use an exposure time of $t=1 \mathrm{~s}$ to acquire the Raman spectrum of a material. The problem arises when a Raman image has to be made. Consider a Raman map made over a $50 \times 50 \mu \mathrm{m}^{2}$ region of the aforementioned d-SA layer. At a resolution of $100 \times 100$ pixels, a Raman amplitude-based image made at an exposure time of $1 \mathrm{~s}$ /pixel will take $\approx 167$ minutes. This is a very long time to obtain necessary spatially-resolved chemical characterization of the probed material. Naturally, it is possible to lower the exposure time/pixel to achieve faster imaging times, but only to the extent of an acceptable SNR of the single-pixel Raman spectrum. A careful consideration of the trade-off between faster imaging times and sufficient SNR is necessary. For example, imaging instead at $50 \mathrm{~ms}$ per pixel to achieve a total imaging time of only 8.3 minutes is possible, but the estimated number of Raman photons drops by a factor of 20 , which would result in a considerably lower SNR. The real spectrum of a $100 \mathrm{~nm}$ thick d-SA layer obtained using a commercial CRM at an exposure time of $50 \mathrm{~ms}$ is shown in Figure 1.2(a). 


\subsection{Motivation - imaging in the low SNR regime}

Until now, it is clear that the low Raman scattering cross-section of most materials hinders their reliable and quantitative characterization using Raman imaging because of the low SNR of the acquired Raman spectrum. According to equation 1.2, we identify three parameters which directly result in a poor spectral SNR:

Poor SNR due to low exposure time $(\mathbf{t})$. The fast, spatially-resolved characterization of a variety of materials necessitates the use of a low exposure time/pixel, preferably in the few tens of $\mathrm{ms}$ range. In the previous section, it was already estimated that for a thin organic layer of $\mathrm{d}-\mathrm{SA}$, a quick Raman image made at $\mathrm{t}=50 \mathrm{~ms} /$ pixel would result in a poor SNR (see Figure 1.2(a)). It is clear that the characteristic bands of $d$ SA (marked with asterisks) can hardly be discerned. Naturally, a Raman image made by integrating this band would also be noisy (Figure 1.2(d)), which would give an inaccurate description of the d-SA distribution (compare with Figure 5.4(c), Chapter $5)$.

Poor SNR due to low laser power $(\mathbf{P}) . \quad$ d-SA is stable to laser powers in the few tens of $\mathrm{mW}$ range, however not all materials can be probed using such a high laser dosage. Some materials may undergo photo-induced physical and chemical modifications, which can compromise the integrity of the sample probed. For example, consider the $2 \mathrm{D}$ material of graphene oxide $(\mathrm{GO})$. Although Raman spectroscopy is one of the most indispensable tools for its characterization, GO is easily reduced by the probing laser even at a laser power of $50 \mu \mathrm{W}$ and an exposure time of $1 \mathrm{~s}^{[13,14]}$. To avoid or maximally mitigate these photo-induced changes, the probing laser dose should be as low as possible. Again, this would lead to a poor SNR as shown in Figure 1.2(b). The broad D and G bands of GO are invisible in this case because the laser dosage used is 500 times lower than typically used. The corresponding Raman image shown in Figure 1.2(e) demonstrates the inability to distinguish the multiple layers of GO (compare with Figure 3.2(c), Chapter 3 ). 
(a)

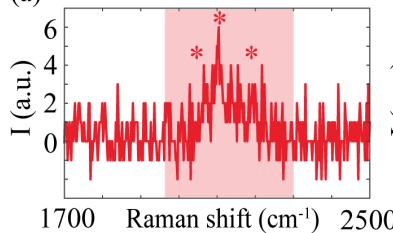

(d)

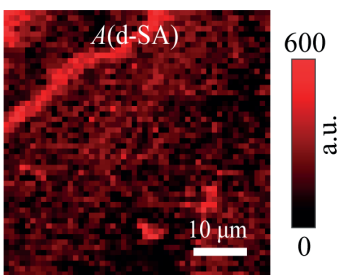

(b)

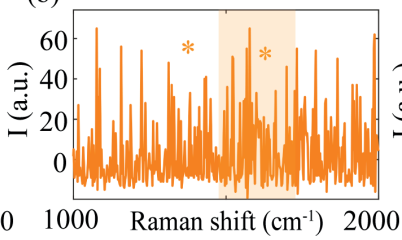

(e)

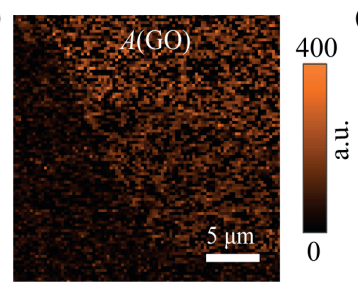

(c)

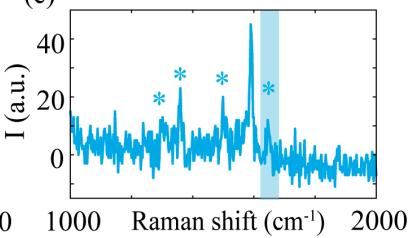

(f)

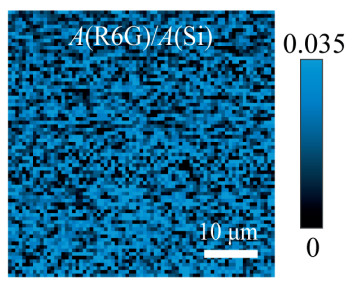

Figure 1.2: Raman spectroscopy and imaging in the low SNR regime. Single-pixel Raman spectrum of (a) a thin layer of $d-S A$, obtained at $P=18 \mathrm{~mW}, t=50 \mathrm{~ms},(b)$ a multi-layer of graphene oxide sheets, obtained at $P=5 \mu \mathrm{W}, t=20 \mathrm{~ms}$, and (c) a monolayer of rhodamine$6 \mathrm{~g}$ adsorbed onto graphene from a bulk solution of concentration $=10 \mathrm{nM}$, obtained at $P=0.25 \mathrm{~mW}$ and $t=100 \mathrm{~ms}$. The characteristic bands of interest for each material is represented by asterisks. (d)-( $f$ ) Raman images obtained by integrating across the band of interest, denoted by the shaded area in their corresponding spectrum (for R6G, this area has been normalized with the silicon band area from the substrate), for every pixel. The low SNR of the spectrum directly translates to noisy Raman images. 
Poor SNR due to low number of probed molecules $(N)$. The Raman intensity is directly proportional to the concentration of species in the probed volume $(N / V)$. Detection of trace amount of analytes adsorbed onto substrates is one of the important applications of Raman spectroscopy, and Raman imaging can provide unprecedented insights into the spatial distribution of such adsorbed layers. Figure 1.2(c) shows the Raman spectrum of rhodamine-6g (R6G) adsorbed onto a monolayer of graphene from a bulk solution of concentration $10 \mathrm{nM}$. At such low bulk concentrations, a partial coverage of the adsorbed monolayer of $\mathrm{R} 6 \mathrm{G}$ is expected ${ }^{[15]}$. Despite the fact that graphenic substrates can enhance the Raman signal of adsorbed molecules via a chemical enhancement mechanism ${ }^{[16]}$, and that the scattering crosssection of conjugated molecules is higher than $\mathrm{d}-\mathrm{SA}^{[17]}$, the resultant SNR of the Raman spectrum of R6G is poor due to the extremely low number of molecules present in the probed volume $\left(\approx 0.0005 \mu \mathrm{m}^{3}\right)$. The resulting distribution of $\mathrm{R} 6 \mathrm{G}$ (Figure 1.2(f)) is also noisy, unlike the image obtained with a better SNR (compare with Figure 4.9(c), Chapter 4).

Thus, in order to successfully implement Raman imaging to study systems in these low throughput scenarios, there is an urgent need to improve the SNR of the collected Raman hyperspectral dataset (HSD). While techniques like surface-enhanced Raman spectroscopy (SERS) ${ }^{[18]}$ could offer better SNR under the same aforementioned conditions, the technique does not provide the same versatility as spontaneous Raman scattering due to the restrictions on the allowed substrates. Moreover, ${ }_{3} \mathrm{D}$ Raman imaging can not be performed in SERS as the field enhancement is highly localized and decays within a few nanometres from the surface ${ }^{[19,20]}$.

\subsection{Thesis outline}

In recent decades, various studies have shown that the problem of insufficient spectral SNR can be resolved by employing data analysis routines which solely rely on mathematical algorithms ${ }^{[21-25]}$. We use one such routine based on principal com- 
ponent analysis (PCA) - a mathematical technique commonly used for dimensionality reduction and feature extraction from a large multi-dimensional dataset ${ }^{\text {[26] }}$ - to improve the SNR of the Raman HSD. The analysis is able to efficiently denoise the Raman spectrum at the single-pixel level by separating out "signal-features" from unwanted "noise-features", thereby automatically improving the quality of the produced Raman images. We denote this PCA-guided denoising technique as "algorithm-improved confocal Raman microscopy (ai-CRM)". In Chapter 2, the underlying mathematics of ai-CRM is explained in a detailed and intuitive manner. Towards the end, the tips and tricks for carrying out a PCA-based denoising of a Raman image dataset is outlined.

The applications of ai-CRM in studying different systems in the low SNR regime is explained in the subsequent chapters. In Chapter 3 , the advantage of ai-CRM in enabling fast characterization of a variety of $2 \mathrm{D}$ materials like graphene, graphene oxide, molybdenum disulfide, tungsten disulfide and boron nitride is delineated. Additionally it is shown that the improved SNR also facilitates non-destructive Raman imaging of graphene oxide - a photo-unstable material that undergoes reduction when probed with conventional laser doses. Finally, the benefit of ai-CRM for fast volumetric Raman imaging is also presented.

Chapter 4 focuses on using ai-CRM to improve the limit of detection of rhodamine$6 \mathrm{~g}$ (R6G) molecules adsorbed onto $2 \mathrm{D}$ graphene oxide analogues. Utilizing the ability of graphenic $2 \mathrm{D}$ materials to enhance the Raman response of adsorbed molecules via a chemical-enhancement mechanism (known as "graphene-enhanced Raman spectroscopy"), coupled with the improved SNR offered by ai-CRM, it is demonstrated that R6G molecules can be detected from bulk aqueous concentrations as low as $5 \times 10^{-10} \mathrm{M}$. Raman imaging further sheds light on the distribution of the adsorbed layer. Lastly, fast in-situ Raman mapping is used to probe the adsorption kinetics of R6G, something which wasn't possible before due to the long times required to acquire even a single Raman image. 
In Chapter 5, ai-CRM enabled fast volumetric mapping is used to monitor the reactive wettability alteration of surfactant coated mica/silica substrates in response to a change in the ambient brine salinity. Orthogonal sectioning of the ${ }_{3} \mathrm{D}$ Raman images (parallel and perpendicular to the substrates) before and after salinity reduction not only helps to visualize the resulting contact angle change of picolitre oil droplets, but also helps in determining the change in material distribution. Finally, based solely on $3 \mathrm{D}$ Raman imaging, a mechanism for this wettability alteration was deduced, which finds its relevance in the field of low salinity flooding based enhanced oil recovery.

\section{Bibliography}

[1] C. V. Raman and K. S. Krishnan, "A new type of secondary radiation," Nature, vol. 121 , no. 3048 , pp. 501-502, 1928.

[2] A. Zoubir, Raman imaging: techniques and applications, vol. 168. Springer, 2012.

[3] L. Nasdala, O. Beyssac, J. W. Schopf, and B. Bleisteiner, Application of Ramanbased images in the Earth sciences, pp. 145-187. Springer, 2012.

[4] A. Paudel, D. Raijada, and J. Rantanen, "Raman spectroscopy in pharmaceutical product design," Advanced drug delivery reviews, vol. 89, pp. 3-20, 2015.

[5] R. Smith, K. L. Wright, and L. Ashton, "Raman spectroscopy: an evolving technique for live cell studies," Analyst, vol. 141, no. 12, pp. 3590-3600, 2016.

[6] W. H. Weber and R. Merlin, Raman scattering in materials science, vol. 42. Springer Science and Business Media, 2013.

[7] M. Ivanda and K. Furić, "Line focusing in micro-Raman spectroscopy," Applied optics, vol. 31, no. 30, pp. 6371-6375, 1992. 
[8] R. W. Havener, S.-Y. Ju, L. Brown, Z. Wang, M. Wojcik, C. S. Ruiz-Vargas, and J. Park, "High-throughput graphene imaging on arbitrary substrates with widefield Raman spectroscopy," ACS Nano, vol. 6, no. 1, pp. 373-38o, 2012.

[9] K. Shaitan and P. Pustoshilov, "Molecular dynamics of a stearic acid monolayer," BIOPHYSICS-PERGAMON THEN MAIK NAUKA-C/C OF BIOFIZIKA, vol. 44, no. 3, pp. 429-434, 1999.

[10] C. A. Lane, D. E. Burton, and C. C. Crabb, "Accurate molecular dimensions from stearic acid monolayers," Journal of Chemical Education, vol. 61, no. 9, p. 815,1984 .

[11] R. C. Prince, R. R. Frontiera, and E. O. Potma, "Stimulated Raman scattering: from bulk to nano," Chemical reviews, vol. 117, no. 7, pp. 5070-5094, 2016.

[12] I. J. Jahn, A. Grjasnow, H. John, K. Weber, J. Popp, and W. Hauswald, “Noise sources and requirements for confocal Raman spectrometers in biosensor applications," Sensors, vol. 21, no. 15, p. 5067, 2021.

[13] S. Nair, J. Gao, Q. Yao, M. H. Duits, C. Otto, and F. Mugele, "Algorithmimproved high speed and non-invasive confocal Raman imaging of twodimensional materials," National Science Review, 2019.

[14] J. S. Mehta, A. C. Faucett, A. Sharma, and J. M. Mativetsky, "How reliable are Raman spectroscopy measurements of graphene oxide?," The Journal of Physical Chemistry C, vol. 121, no. 30, pp. 16584-16591, 2017.

[15] X. Ling, L. Xie, Y. Fang, H. Xu, H. Zhang, J. Kong, M. S. Dresselhaus, J. Zhang, and Z. Liu, "Can graphene be used as a substrate for Raman enhancement?," Nano letters, vol. 10, no. 2, pp. 553-561, 2010.

[16] X. Ling and J. Zhang, "First-layer effect in graphene-enhanced Raman scattering," Small, vol. 6, no. 18, pp. 2020-2025, 2010. 
[17] S. Shim, C. M. Stuart, and R. A. Mathies, "Resonance Raman cross-sections and vibronic analysis of rhodamine $6 \mathrm{~g}$ from broadband stimulated Raman spectroscopy," ChemPhysChem, vol. 9, no. 5, pp. 697-699, 2008.

[18] A. Campion and P. Kambhampati, "Surface-enhanced Raman scattering," Chemical society reviews, vol. 27, no. 4, pp. 241-250, 1998.

[19] A. Otto, "Theory of first layer and single molecule surface enhanced Raman scattering (SERS)," physica status solidi (a), vol. 188, no. 4, pp. 1455-1470, 2001.

[20] G. Kovacs, R. Loutfy, P. Vincett, C. Jennings, and R. Aroca, “Distance dependence of SERS enhancement factor from Langmuir-Blodgett monolayers on metal island films: evidence for the electromagnetic mechanism," Langmuir, vol. 2, no. 6, pp. 689-694, 1986.

[21] M. Unser and A. Aldroubi, "A review of wavelets in biomedical applications," Proceedings of the IEEE, vol. 84, no. 4, pp. 626-638, 1996.

[22] D. Shen, G. Wu, and H.-I. Suk, "Deep learning in medical image analysis," Annual review of biomedical engineering, vol. 19, pp. 221-248, 2017.

[23] C. Kallepitis, M. S. Bergholt, M. M. Mazo, V. Leonardo, S. C. Skaalure, S. A. Maynard, and M. M. Stevens, "Quantitative volumetric Raman imaging of three dimensional cell cultures," Nature communications, vol. 8, p. 14843, 2017.

[24] C. C. Horgan, M. Jensen, A. Nagelkerke, J.-P. St-Pierre, T. Vercauteren, M. M. Stevens, and M. S. Bergholt, "High-throughput molecular imaging via deep learning enabled Raman spectroscopy," arXiv preprint arXiv:2009.13318, 2020.

[25] R. Gautam, S. Vanga, F. Ariese, and S. Umapathy, "Review of multidimensional data processing approaches for Raman and infrared spectroscopy," EPJ Techniques and Instrumentation, vol. 2, no. 1, pp. 1-38, 2015. 
[26] H. Abdi and L. J. Williams, "Principal component analysis," Wiley interdisciplinary reviews: computational statistics, vol. 2, no. 4, pp. 433-459, 2010. 
"We don't have better algorithms, we just have more data."

Peter Norvig

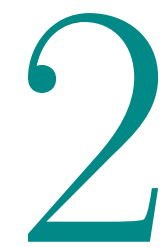

\section{Algorithm-improved confocal Raman microscopy: principle and technique}

IN THIS CHAPTER I will focus on the underlying mathematics of algorithm-improved confocal Raman microscopy (ai-CRM), a technique which has been used throughout this thesis to improve the signal-to-noise ratio (SNR) of the collected Raman hyperspectral dataset. Additionally, the methodology for implementing ai-CRM for fast and non-invasive $2 \mathrm{D}$ and $3 \mathrm{D}$ Raman imaging is outlined. 


\subsection{Confocal Raman microscopy (CRM)}

Typically, Raman microscopy or Raman micro-spectroscopy refers to the usage of an optical microscope to carry out spectroscopic characterization of materials. The major components of a Raman microscope are:

1. An excitation source (laser)

2. Optical microscope

3. Spectrometer

4. Detector

\section{Confocal Raman setup}

All Raman measurements mentioned in this thesis were carried out using a commercial WITec alpha 30oR Raman microscope (WITec GmbH, Ulm, Germany). The schematic of the Raman setup is shown in Figure 2.1. A $532 \mathrm{~nm}$ diode-pumped solid-state (DPSS) laser is used as the excitation source, with a maximum power at output measured at $\sim 47 \mathrm{~mW}$. The laser beam is then guided to the microscope via an optical fibre and focused onto the substrate through one of the following objectives: a 100x/0.9 NA/0.31 mm WD (Zeiss EC “Epiplan-Neofluar” DIC, Carl Zeiss) or a $63 \mathrm{x} / 0.9 \mathrm{NA} / 2.4 \mathrm{~mm}$ WD water-dipping (Zeiss W"N-Achroplan”, Carl Zeiss) objective, depending on the necessity. White-field illumination is provided separately through the same objective using a switchable filter. The substrate is placed on a motorized sample scanning stage, which has a travel range of $50 \times 50 \mathrm{~mm}^{2}$ and a minimum spatial (x-y) step size of $100 \mathrm{~nm}$. The focus is adjusted by moving the objective in the axial direction $(\mathrm{z})$.

The scattered photons are collected via the same objective in a back-scattering configuration. The photons then pass through a dichroic beam splitter and a Rayleigh line rejection filter. The filtered light is guided to the output optical fibre - the open- 
ing of which acts as a pinhole and provides confocality by reducing the out of focus light. This provides an improved resolution in the axial direction $(\mathrm{z})$ as compared to non-confocal microscopes, enabling sequential depth-wise optical sectioning of the sample useful for ${ }_{3} \mathrm{D}$ or volumetric Raman imaging. Next, the light reaches the spectrometer, with a diffraction grating as the dispersive element. A diffraction grating of density $600 \mathrm{~g} / \mathrm{mm}$ or $1800 \mathrm{~g} / \mathrm{mm}$ was used. The dispersed light finally falls on a (electron-multiplying) charge-coupled device (CCD, $1600 \times 200$ pixels, $16 \mu \mathrm{m}$ pixel size, Andor Newton) camera, which is thermo-electrically cooled down to $-60^{\circ} \mathrm{C}$ prior to measurements. The camera is either operated in the CCD mode (EMCCD gain value $=0$ ) or the EMCCD mode $($ EMCCD gain value $=250$ (maximum allowed by the system), pre-amplifier gain value $=1$ ). The Raman spectrum is then displayed on the computer through the WITec Control software.

\section{Data acquisition}

A typical Raman scan is made by specifying the following parameters prior to the scan:

1. Laser power at objective $(\mathrm{mW})$

2. Detector mode (CCD or EMCCD)

3. Type of grating $(600 \mathrm{~g} / \mathrm{mm}$ or $1800 \mathrm{~g} / \mathrm{mm})$

4. Dimensions of the region probed $(\mu \mathrm{m}(\mathrm{x}) \times \mu \mathrm{m}(\mathrm{y}) \times \mu \mathrm{m}(\mathrm{z}))$

5. Resolution of the region probed $(\operatorname{pixels}(\mathrm{x}) \times \operatorname{pixels}(\mathrm{y}) \times \operatorname{pixels}(\mathrm{z}))$

6. Integration/exposure time per pixel (s)

These parameters are specified accordingly in each chapter. The Raman microscope then performs a scan by rasterisation - acquiring a spectrum (Intensity (a.u.) vs. Raman shift $\left.\left(\mathrm{cm}^{-1}\right)\right)$ at each pixel in a line-by-line fashion - according to the resolution 
(f)

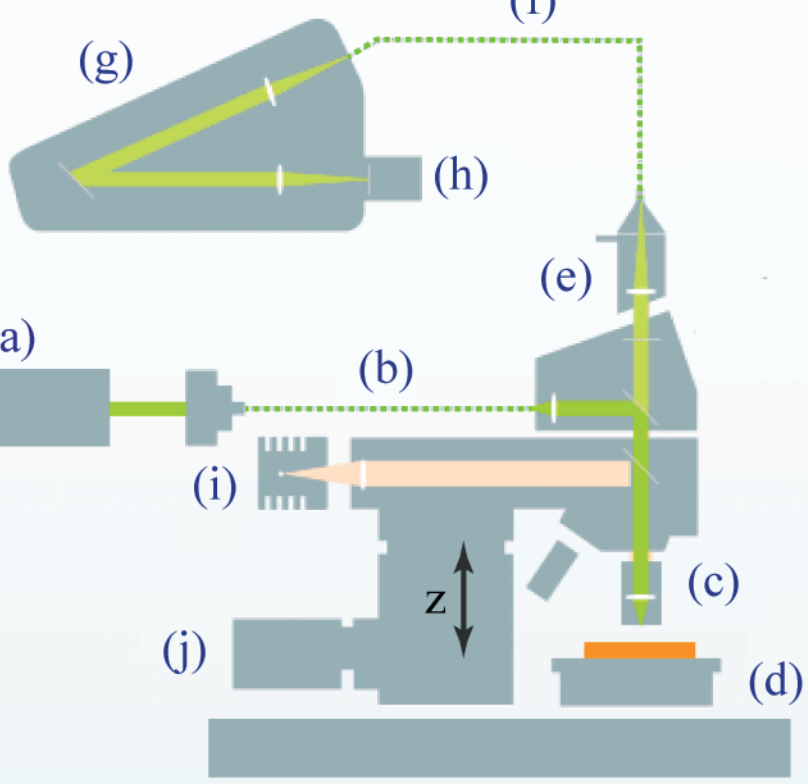

Figure 2.1: Schematic of the confocal Raman microscope used in this study. (a) $532 \mathrm{~nm}$ excitation laser, (b) input optical fibre, (c) objective turret, (d) sample + motorized sample stage, (e) filters, $(f)$ output optical fibre, $(g)$ spectrometer, $(h)$ EM(CCD), (i) white-light illumination and $(j)$ motorized $z$-control for focussing. Adapted from ${ }^{[1]}$. 
provided. The final acquired data is stored as a matrix containing the Raman spectrum vs. pixel number. Henceforth, this matrix will be referred to as the hyperspectral dataset (HSD).

\section{Cosmic ray removal}

Cosmic rays impinging onto the CCD can cause high intensity (usually way higher than the Raman bands), narrow width (1-4 pixels) spikes in the acquired HSD. These spikes are removed from the HSD using the "Cosmic ray removal (CRR)" option in the WITec Project FIVE+ software.

\section{Baseline correction}

The presence of fluorescent molecules or impurities can interfere with the Raman spectrum, which appears as a broadband, curved baseline which changes with time. The main challenge is to extract signal features from the Raman spectrum with a dominant fluorescent background, because the latter is often orders of magnitude stronger than Raman scattering. Background subtraction can help flatten this baseline, helping in the identification and analysis of bands which would otherwise be difficult to quantify due to the poor signal-to-noise ratio (SNR) of the fluorescent baseline. If necessary, background correction of the HSD is performed on the WITec Project FIVE+ software, which has an option for background subtraction using different functions. It was found that the "shape" option for baseline subtraction is highly efficient as compared to the "polynomial" option (which fits an $n^{\text {th }}$ order polynomial to the baseline) in the WITec Project FIVE+ software.

\subsection{Algorithm-improved CRM (ai-CRM)}

Following the data acquisition and pre-treatment steps described above, the HSD is exported as a matrix - the first column of which corresponds to wavenumber data points, and every successive column corresponds to the intensity values (at every 
wavenumber) at each pixel. As explained in Chapter 1, Raman scattering is inherently a weak phenomenon (scattering cross-section $\approx 10^{-34} \mathrm{~m}^{2} / \mathrm{sr}$ ), and conventionally obtained HSDs suffer from poor SNR if the requirement of a high-resolution, fast and non-invasive Raman map of the sample puts a limit on the maximum probing laser dosage. Naturally, this low SNR can make reliable quantification and Raman imaging difficult, as shown in Figure 1.2. To circumvent this problem and improve the SNR, we developed ai-CRM, which relies on efficient denoising of the HSD by subjecting it to the mathematical techniques of singular value decomposition and principal component analysis, which are explained in detail in the following sections.

\subsubsection{Singular Value Decomposition}

The underlying concept of principal component analysis (PCA) is based on a very important matrix factorization technique in linear algebra known as singular value decomposition (SVD). Given any matrix $A_{m \times n}$ (where $m$ is the number of measurements or samples, $n$ the number of variables and $A$ is zero-mean centered), SVD decomposes it into a product of three matrices as follows:

$$
A=U \Sigma V^{T}
$$

Here, $U$ and $V$ are orthogonal matrices $\left(\therefore V^{T} V=I, U^{T} U=I\right)$ of dimension $m \times m$ and $n \times n$ respectively and $\Sigma$ is a diagonal matrix of dimensions $m \times n$ (the nonsquare diagonal elements of $\Sigma$ are zero). Before going into the details of how these matrices are determined, it is important to understand the graphical interpretation of SVD.

Graphical interpretation. Consider a vector $a=\left(\begin{array}{ll}a_{x} & a_{y}\end{array}\right)$ that is projected onto a set of orthogonal axes $v_{1}$ and $v_{2}$ as shown in Figure 2.2(a). The lengths of projection 
(a) 2

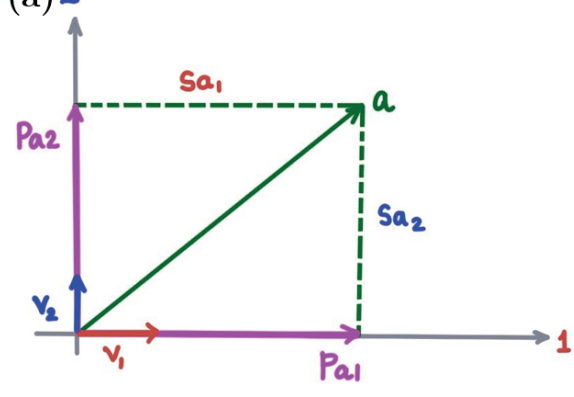

(b)

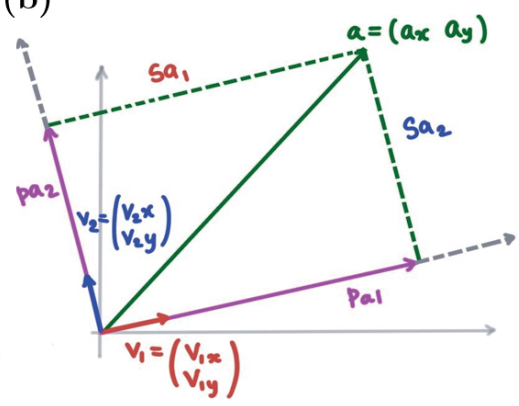

Figure 2.2: (a) Projection of a two-dimensional vector "a" on a set of standard orthogonal axes and (b) generalized projection of " $a$ " on a set of random orthogonal axes.

on the two axes are $S_{a 1}=a \cdot v_{1}$ and $S_{a 2}=a \cdot v_{2}$, respectively. This relation readily extends to any set of orthogonal axes, $v_{1}=\left(v_{1 x}, v_{1 y}\right)$ and $v_{2}=\left(v_{2 x}, v_{2 y}\right)$ (Figure 2.2(b)), as well as any number of vectors (for simplicity, we consider another twodimensional vector $\left.b=\left(\begin{array}{ll}b_{x} & b_{y}\end{array}\right)\right)$, and in general the projected length matrix $S$ can be written as:

$$
\begin{gathered}
{\left[\begin{array}{ll}
S_{a_{1}} & S_{a_{2}} \\
S_{b_{1}} & S_{b_{2}}
\end{array}\right]=} \\
=\left[\begin{array}{ll}
a_{x} & a_{y} \\
b_{x} & b_{y}
\end{array}\right]\left[\begin{array}{ll}
v_{1 x} & v_{2 x} \\
v_{1 y} & v_{2 y}
\end{array}\right] \\
S=A V
\end{gathered}
$$

Since the columns of $V$ are orthonormal, equation $2.2 \mathrm{~b}$ can be rearranged as:

$$
A=S V^{-1}=S V^{T}
$$

Next, we focus on the matrix $S$. It is possible to normalize the elements of $S$ with their 
corresponding column lengths, $l_{1}$ and $l_{2}$, as shown below, such that $S$ can be expressed as a product of two matrices - one orthonormal and one diagonal as follows:

$$
S=\left[\begin{array}{ll}
S_{a 1} & S_{a 2} \\
S_{b_{1}} & S_{b_{2}}
\end{array}\right]=\left[\begin{array}{cc}
\frac{S_{a 1}}{l_{1}} & \frac{S_{a 2}}{l_{2}} \\
\frac{S_{b_{1}}}{l_{1}} & \frac{S_{b_{2}}}{l_{2}}
\end{array}\right]\left[\begin{array}{ll}
l_{1} & 0 \\
0 & l_{2}
\end{array}\right]=U \Sigma
$$

Substituting equation 2.4 in equation 2.3, we get:

$$
A=U \Sigma V^{T}
$$

which is an example of the SVD of a matrix A. Understanding SVD graphically gives us an intuition of the decomposition in 2 or 3 dimensions. In reality, the decomposition can be performed on any matrix $A$ of dimensions $\mathrm{m} \times \mathrm{n}$. In the following sections, the method to determine the matrices $U, \Sigma$ and $V$ will be explained and additionally, the benefit of looking at the data in projected space $S$ will be elucidated.

Determining matrices $U, \Sigma$ and $V$. In order to determine the three matrices resulting from the SVD of matrix $A$, one has to evaluate the covariance matrix $C$ of $A$, defined as follows:

$$
C_{A}=\frac{1}{m-1} A^{T} A
$$

Here, $C_{A}$ is a square-symmetric matrix which is at least positive semi-definite. By definition, this implies that the eigenvectors of $C_{A}$ can be chosen to be orthonormal, whereas the eigenvalues of $C_{A}$ are at least zero or positive. This is an important property, and in the subsequent sections it will be clear how this property works in favour of PCA-based dimensionality reduction. The diagonal elements of this matrix describe the variance within individual variables and the off-diagonal elements 
contain the corresponding covariance between any two variables of $A$. Substituting 2.1 in 2.6, $C_{A}$ is reduced to:

$$
\begin{gathered}
C_{A}=\frac{1}{m-1}\left(U \Sigma V^{T}\right)^{T}\left(U \Sigma V^{T}\right) \\
C_{A}=\frac{1}{m-1} V \Sigma^{2} V^{T}
\end{gathered}
$$

Equation $2.7 \mathrm{~b}$ is analogous to the eigendecomposition of a symmetric matrix. As a result, the columns of $V$ contain the orthonormal eigenvectors and the diagonal elements of $\Sigma^{2}$ contain the eigenvalues $\left(\lambda_{i} \geq 0\right)$ of $C_{A}$. In order to determine $U$, we just need to define the covariance of $A^{T}$, i.e., $C_{A^{T}}=\frac{1}{m-1} A A^{T}$ and substitute $A$ from equation 2.1 like before. On simplification, we get:

$$
C_{A^{T}}=\frac{1}{m-1} U \Sigma^{2} U^{T}
$$

This is analogous to the eigendecomposition of $C_{A^{T}}$, and therefore, the columns of $U$ contain the orthonormal eigenvectors and $\Sigma^{2}$ contains the eigenvalues of $C_{A^{T}}$. Note that the eigenvalues of both $C_{A}$ and $C_{A^{T}}$ are the same. We define the square root of the eigenvalues $\left(\lambda_{i}\right)$ as the singular values, $\sigma_{r}(r=\min (m, n))$, which are therefore the diagonal entries of $\Sigma$.

In summary, equation 2.1 can be denoted in matrix form as shown below: 


$$
\left[\begin{array}{ccc}
a_{11} & \ldots & a_{1 n} \\
\vdots & \ddots & \vdots \\
a_{m 1} & \ldots & a_{m n}
\end{array}\right]_{m x n}=\left[\begin{array}{lll}
u_{1} & \ldots & u_{m}
\end{array}\right]_{m x m}\left[\begin{array}{ccc}
\sigma_{1} & \ldots & 0 \\
\vdots & \ddots & \vdots \\
0 & \ldots & \sigma_{r}
\end{array}\right]_{m x n}\left[\begin{array}{lll}
v_{1} & \ldots & v_{n}
\end{array}\right]_{n x n}^{T}
$$

Here, $a_{i j}$ are the elements of the data matrix $A, u_{i}$ are the eigenvectors of $C_{A^{T}}, \sigma_{r}$ are the sqaure roots of the eigenvalues of $C_{A}\left(\right.$ or $C_{A^{T}}$ ) and $v_{j}$ are the eigenvectors of $C_{A}$, wherein $i=1,2 \ldots m ; j=1,2 \ldots n$ and $r=\min (m, n)$. The singular values can be chosen to be in decreasing order of their magnitude, i.e., $\sigma_{1} \geq \sigma_{2} \geq \ldots \geq \sigma_{r} \geq 0$ in $\Sigma$. The vectors $u_{i}$ and $v_{j}$ are also referred to as the left and right singular vectors in the SVD.

Data in projected space $S$. The covariance matrix of matrix $A$, denoted by equation 2.6, gives detailed information of the variation in the data. As mentioned before, the diagonal elements contain the variance within each variable, which determines the amount of information or structure stored in that variable. Higher variance describes more contribution to the overall data described by the variable, whereas low variance variables contribute less. The off-diagonal elements denote the covariance, which is an indicator of dependency between variables. Higher values of covariance denote redundancy in the data, whereas a low covariance denotes that the two variables are relatively uncorrelated. In the given set of coordinate axes (basis vectors), the covariance matrix exposes this structure and redundancy between variables. However, there can exist another set of basis vectors, which maximizes the variance along those axes while simultaneously minimizing the covariance to zero. In the section on graphical interpretation of SVD, the concept of projected space $S$ was mentioned, which was basically the projection of the dataset onto any random set of basis vectors. As an example, let this new set of basis vectors be specifically $V$ (the same as obtained from the SVD of $A$ ). Then the projected space, $S$ is given 
by:

$$
S=A V
$$

Now, let us calculate the covariance matrix of this projected space, $S$ as below:

$$
C_{S}=\frac{1}{m-1} S^{T} S=\frac{1}{m-1}(A V)^{T}(A V)
$$

From equation 2.1, we know that $A V=U \Sigma$. Substituting this in the above equation, we get:

$$
C_{S}=\frac{1}{m-1}\left(\Sigma^{T} U^{T}\right)(U \Sigma)=\frac{1}{m-1} \Sigma^{2}
$$

As a consequence of viewing the original data $A$ in projected space, the covariance matrix is now diagonalized (the off-diagonal elements are zero). This transformation highlights the importance of SVD as a powerful matrix decomposition technique. The decomposition directly provides with a new set of basis vectors $V$, which can be used to handle data better in the projected space $S$ with minimum redundancy. Additionally, SVD also provides with the singular values of $A$, which lays a basis for dimensionality reduction using principal component analysis as explained in the section below.

\subsubsection{Principal Component Analysis}

PCA is a mathematical technique which transforms a set of possibly correlated variables to a set of linearly independent variables known as the principal components. These principal components are simply a new set of basis vectors (orthogonal coordinate axes) along which the maximum variation in the data is captured. Note that the SVD of a matrix $A$ directly gives us these directions $\left(v_{i}\right)$, as shown in the 
previous section. PCA is widely used as a dimensionality reduction technique while still preserving maximum information of the original data. The way PCA recognizes the $k$ most important components can be realized by observing the singular values $\sigma_{r}$. Higher singular values describe most of the variance captured in the direction of the corresponding principal components. Lower singular values contribute less to the overall structure of the data. The overall variance of the data in projected space can be determined by the trace of $C_{S}$, as shown below:

$$
\text { overall variance }=\operatorname{trace}\left(C_{S}\right)=\sigma_{1}^{2}+\sigma_{2}^{2}+\ldots+\sigma_{r}^{2}
$$

Note that the sum on the right is also equivalent to the sum of the eigenvalues $\left(\lambda_{i}\right)$ of the covariance matrix of $A$. Since the singular values can be chosen to be arranged in decreasing order of their magnitude, the initial principal components $\left(v_{i}\right)$ account for most of the variance in the data. It is now possible to determine the contribution of any component to the overall variance as follows:

$$
\% \text { explained variance } \mathrm{r}_{\mathrm{r}}=\frac{\sigma_{r}^{2}}{\text { overall variance }} \times 100
$$

To determine the number of retained components $k$, a common technique used is known as the Scree test. The procedure involves plotting the explained variance of each component with the corresponding principal component number (equation 2.14), which is known as the Scree plot. Usually, this results in an "elbow" shaped graph, wherein the variance drops rapidly in the initial few components, slowly tailing off to the lower explained variance values. The minimum number of statistically relevant components that should be retained, $k$ should be at least at the aforementioned "elbow" point. An example of the Scree test is provided in a later section on using PCA-based denoising of Raman HSDs.

By keeping only the most important principal components, PCA helps in reducing 
the dimensionality of the original data matrix $A$, from $n$ variables to $k$ variables. Note that the variables themselves do not vanish, because the $k$ variables in projected space $(S)$ are actually linear combinations of the $n$ initial variables. Expanding the general matrix formulation of SVD of matrix $A$, as shown in equation 2.9, gives us:

$$
A=U \Sigma V^{T}=\sigma_{1} U_{1} V_{1}^{T}+\sigma_{2} U_{2} V_{2}^{T}+\ldots+\sigma_{r} U_{r} V_{r}^{T}
$$

It is now possible to approximate the original data, by using only the $k$ leading singular values as follows:

$$
\tilde{A}=U_{k} \Sigma_{k} V_{k}^{T}=\sigma_{1} U_{1} V_{1}^{T}+\sigma_{2} U_{2} V_{2}^{T}+\ldots+\sigma_{k} U_{k} V_{k}^{T}
$$

It is important to note here that this reconstruction of the original data is lossy and depends on the number of retained components. The leading $k$ singular values explain most of the variation in the data, and hence leaving them out during reconstruction would naturally lead to a poor reconstruction. The remaining $r-k$ singular values may be dropped or set to zero without affecting the reconstruction error significantly. This idea is exploited to achieve efficient denoising of Raman HSDs to drastically improve their SNR, and is explained in the following section.

\subsubsection{PCA-based denoising of Raman HSD}

Until now, it is clear that the SVD of any data matrix $A_{m \times n}$ gives us its singular values $\left(\sigma_{r}\right)$ as well as an orthogonal set of vectors $\left(v_{i}\right)$, which are linearly uncorrelated directions corresponding to those singular values. Then, PCA is used to define a new set of basis vectors ( principal components, $v_{i}$ ) along which maximum variation of the data is captured. Dimensionality reduction is achieved by projecting $A$ onto the leading $k$ principal components, wherein the latter is estimated by using a Scree plot. Finally, data reconstruction is made possible by back-calculating the data matrix $\tilde{A}_{m \times n}$, but 
by retaining only the $k$ leading singular values and setting the remaining singular values $\left(\sigma_{k+1}\right.$ to $\left.\sigma_{r}\right)$ to zero.

Below, we explain how the technique of PCA is readily extended for denoising a large Raman HSD (for e.g., obtained by performing 2D/3D Raman imaging):

1. The Raman data is arranged in the form of a matrix $A_{m \times n}$, where $\mathrm{m}$ is the number of measurements (number of pixels) and $\mathrm{n}$ is the number of variables (wavenumber data points). Therefore, each row of this matrix corresponds to a Raman spectrum obtained at a particular pixel. This matrix is zero-mean centered. This is an important step, as the first principal component will correspond to the direction of maximum variation of the data only if the data is zero-mean centered. If the data is not zero-mean centered, then the first principal component will roughly correspond to the mean of the data itself, which can be misleading.

2. This matrix is subjected to a singular value decomposition (equation 2.1) and the left and right singular vectors (columns of $U$ and $V$, respectively) and singular values (diagonal elements of $\Sigma$ ) are determined. Note that the singular values are chosen to be in decreasing order of their magnitude $\left(\sigma_{r} \geq 0\right)$ along the diagonal elements. The corresponding columns of $V\left(v_{i}\right)$ are known as the principal components. The full projected space, $S$ can be calculated using equation 2.10 and this is also known as the scores of the principal components.

3. PCA distinguishes signal and noise features by their different collective behaviours. Since noise is largely random, its contribution to the variance in different components are relatively constant and low (black line, Figure 2.3(a)). However, signals are a recurring feature in the HSD and their contribution to the variance will accumulate to a high value (red curve, Figure 2.3(a)). PCA ranks the components according to variance (singular values), and therefore, 
(a)

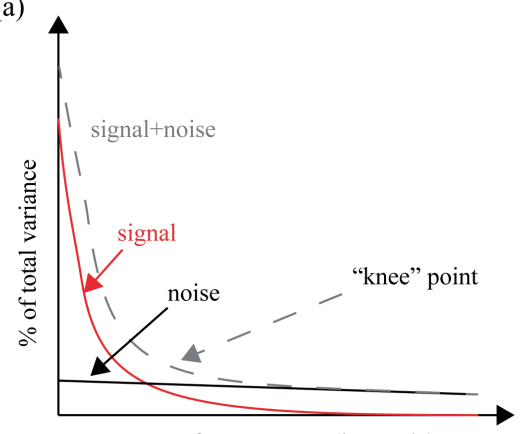

No. of components (log scale) (b)

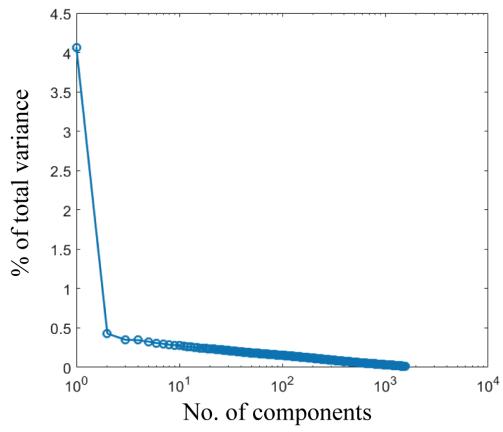

Figure 2.3: (a) Schematic of a Scree plot showing the contributions of "signal-" and "noisecomponents" and (b) Actual Scree plot of a PCA analysis on a Raman HSD shown in Chapter 3, Figure 3.2(c).

the signals will be captured in the leading $k$ principal components. When the variances start to decrease slowly, mostly noise is captured. To select the "signal-components", a Scree test is used. The percentage of total variance contributed by each component (equation 2.14) is plotted for all components. Then we find a "knee" point in the plot. Selecting a few more components on the right side of the knee point does not affect the denoising performance since most of the noise is rejected anyway. A real example of a Scree plot is shown in Figure 2.3(b), which corresponds to the Raman HSD shown in Chapter 3, Figure 3.2(c).

4. After determining the appropriate number $k$ of singular values that explains most of the variance in the data, the original data is reconstructed according to equation 2.16. This is the denoised data matrix, $\tilde{A}$. As mentioned before, the quality of reconstruction and denoising depends on the number of singular values retained. Choosing too few singular values $(k=1,2)$ in the "signalcomponents" regime can result in amplitude loss of the Raman bands during 
reconstruction. Choosing too many will improve the reconstruction error, but in the context of Raman denoising, the HSD will end up resembling more like the original noisy data. An example of the dependence of the reconstructed spectrum on the number of principal components (or singular values) retained, corresponding to the Raman HSD shown in Chapter 3, Figure 3.2(c) is shown in Figure 2.4.

5. The denoised data matrix $\tilde{A}$ shows a drastically improved SNR as compared to the original Raman HSD, A. Raman imaging can now be performed by integrating across the band of interest (or other types of mapping like band amplitude, band positions etc.).

This technique of using PCA-based denoising is what we term as algorithmimproved confocal Raman microscopy (ai-CRM). Various other techniques like smoothing or filtering can be used to improve the SNR of individual Raman spectrum $^{[2-4]}$. However, these techniques require substantial tweaking of the smoothing/filtering parameters so as to ensure good noise elimination and minimal band amplitude loss. Moreover, when a large number of measurements are made in the low SNR regime, it is highly impractical and computationally expensive to perform quality checks for each and every Raman spectrum in the HSD. Thus, there is a need to adopt a denoising technique which can not only handle large datasets, but is also robust and problem-independent. Multivariate data analysis techniques like PCA can be used for efficient feature extraction and classification. PCA looks at the entire structure of the HSD as a whole, and can efficiently reject unwanted noise components as explained above. Additionally, since the principal components can be readily computed by performing a SVD of the data matrix, which is a well-established routine in a lot of computational platforms like MATLAB, denoising 10,000 spectra at a time is fast and takes an average of $20 \mathrm{~s}$ on a normal office computer. Finally, PCA requires the tweaking of only $k$, i.e., the number of singular values retained during reconstruction, which again can be easily determined by using the Scree plot. 


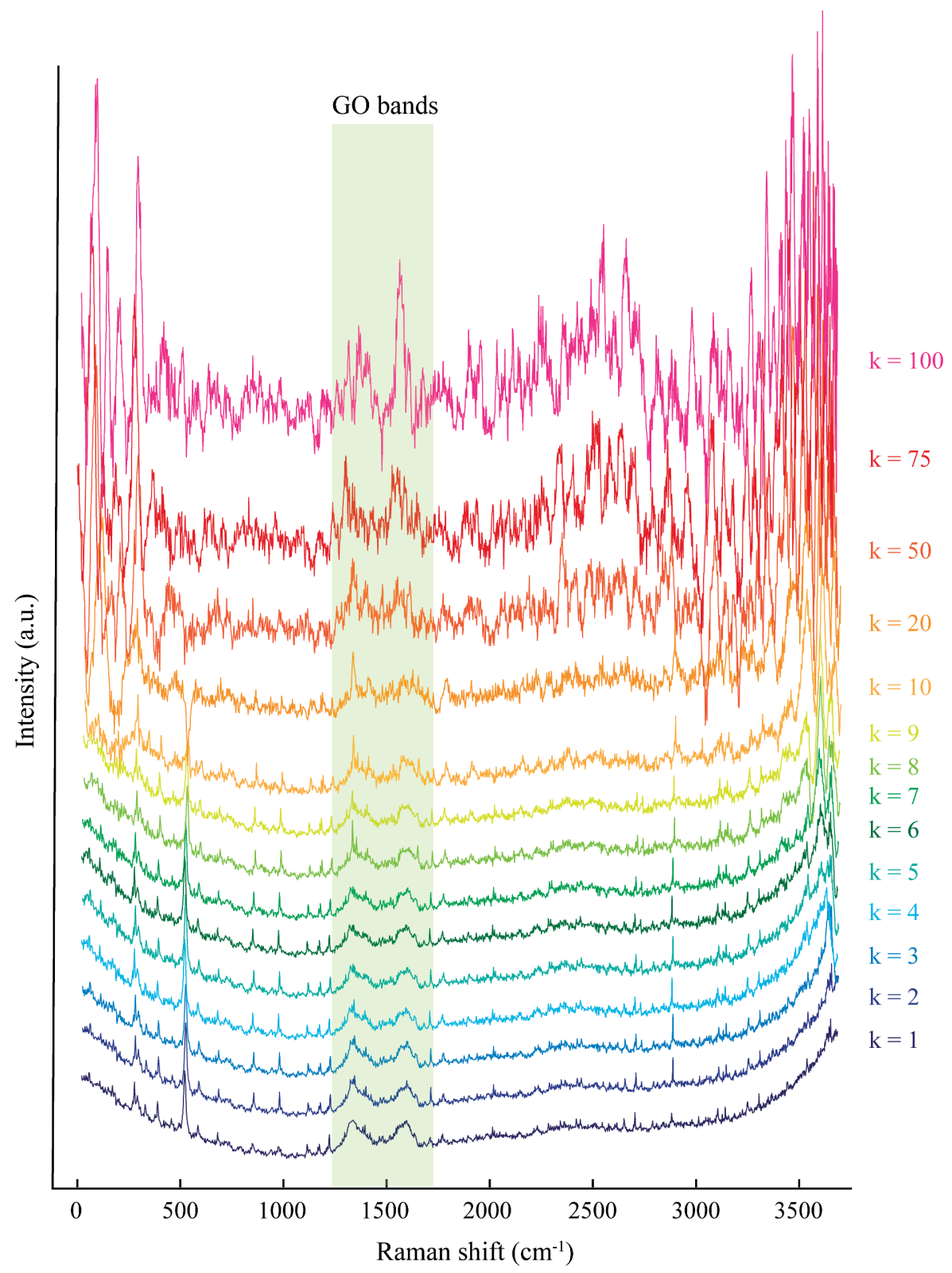

Figure 2.4: Evolution of the denoised single pixel spectrum as a function of the first $k$ components retained for reconstruction corresponding to the Raman HSD depicted in Chapter 3, Figure 3.2 (c). The spectral SNR of the D and G bands of graphene oxide $\left(\right.$ at $1352 \mathrm{~cm}^{-1}$ and $1597 \mathrm{~cm}^{-1}$, respectively) starts becoming worse, especially after $k=10$. 
Considering all these advantages, ai-CRM has been extensively used throughout all the subsequent chapters in this thesis to improve the SNR of Raman data. A variety of systems have been studied wherein the limitation on the maximum probed laser dosage results in low SNR of acquired the Raman data. This includes the requirements of fast and non-invasive spatial mapping of $2 \mathrm{D}$ materials (low SNR due to low integration time and laser power used), low limit of detection and imaging of analyte molecules on $2 \mathrm{D}$ materials (low SNR due to sub-nM analyte concentrations) as well as fast $3 \mathrm{D}$ mapping (low SNR due to fast acquisition of a large-sized HSD).

\section{Bibliography}

[1] M. Villà Montero, "Laser heating and destruction of carbon nanotubes," B.S. thesis, Universitat Politècnica de Catalunya, 2013.

[2] M. Člupek, P. Matějka, and K. Volka, "Noise reduction in raman spectra: Finite impulse response filtration versus savitzky-golay smoothing," Journal of Raman Spectroscopy: An International Journal for Original Work in all Aspects of Raman Spectroscopy, Including Higher Order Processes, and also Brillouin and Rayleigh Scattering, vol. 38, no. 9, pp. 1174-1179, 2007.

[3] F. Ehrentreich and L. Sümmchen, "Spike removal and denoising of raman spectra by wavelet transform methods," Analytical chemistry, vol. 73, no. 17, pp. 4364-4373, 2001.

[4] S. Chen, X. Lin, C. Yuen, S. Padmanabhan, R. W. Beuerman, and Q. Liu, "Recovery of raman spectra with low signal-to-noise ratio using wiener estimation," Optics Express, vol. 22, no. 10, pp. 12102-12114, 2014. 
"I'm considering whether or not to believe you. I need to run an algorithm on this."

Jessica Park

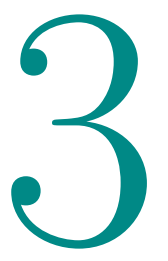

\section{Algorithm-improved high speed and non-invasive confocal Raman imaging of two-dimensional materials ${ }^{\dagger}$}

CONFOCAL RAMAN MICROSCOPY is important for characterizing two dimensional (2D) materials, but its low throughput significantly hinders its applications. For metastable materials such as graphene oxide (GO), the low throughput is aggravated by the requirement of an extremely low laser dose to avoid sample damage. Here we

\footnotetext{
${ }^{\dagger}$ Published as: Nair, Sachin et al., National Science Review 7.3 (2020): 620-628.
} 
introduce algorithm-improved Confocal Raman Microscopy (ai-CRM), which increases the Raman scanning rate by one to two orders of magnitude with respect to state-of-the-art works for a variety of $2 \mathrm{D}$ materials. Meanwhile, GO can be imaged at a laser dose that is 2 to 3 orders of magnitude lower than previously reported, such that laser-induced variations of the material properties can be avoided. ai-CRM also enables fast and spatially resolved quantitative analysis, and is readily extended to three-dimensional mapping of composite materials. Since ai-CRM is based on general mathematical principles, it is cost-effective, facile-to-implement and universally applicable to other hyperspectral imaging methods.

\subsection{Introduction}

Confocal Raman microscopy (CRM) has become one of the most widely used analytical methods to investigate the physico-chemical properties of two-dimensional (2D) materials ${ }^{[1,2]}$. Compared to other methods such as optical microscopy ${ }^{[3-6]}$, atomic force microscopy ${ }^{[3,7]}$, and fluorescence quenching microscopy ${ }^{[8,9]}$, Raman microscopy has the advantage to provide label-free, spatially resolved, compositional and structural information of the probed material on arbitrary substrates. Raman microscopy has enabled studies on the quality ${ }^{[10]}$, defect ${ }^{[11]}$, number of layers ${ }^{[10,12]}$, crystal edges $^{[13]}$, strain ${ }^{[5]}$, oxidation state ${ }^{[14]}$ and electron-phonon coupling ${ }^{[15]}$ of $2 \mathrm{D}$ materials. For example, to determine the quality of fabricated graphene, it is common to present the characteristic Raman spectrum showing an intense G' peak $\left(\approx 2700 \mathrm{~cm}^{-1}\right)$, which is also referred to as the $2 \mathrm{D}$ peak (to avoid confusion with “two-dimensional”, we denote it as $\left.\mathrm{G}^{\prime}\right)$, together with a weak or absent $\mathrm{D}$ band $(\approx$ $\left.1350 \mathrm{~cm}^{-1}\right)^{[10,16]}$. The quality can be further quantified by comparing the ratio of $\mathrm{G}$ and $D$ band intensity ${ }^{[17]}$. For graphene oxide (GO), the changes in the intensity and peak position of the $\mathrm{D}$ and the $\mathrm{G}\left(\approx 1600 \mathrm{~cm}^{-1}\right)$ band can be used to characterize its thermal reduction behavior ${ }^{[18]}$. For many other $2 \mathrm{D}$ materials such as $\mathrm{BN}^{[19,20]}$, transition metal dichalcogenides ${ }^{[2,21,22]}$ and phosphorene ${ }^{[23]}$, Raman microscopy 
has also become an important characterization tool.

However, the potential of Raman microscopy is severely hindered by its low throughput, due to the extremely low efficiency of the Raman scattering cross section: on average, 1 out of 10 million incident photons are Raman scattered. Typically, a single Raman spectrum of graphene takes hundreds of milliseconds to tens of seconds to acquire ${ }^{[24-30]}$. Consequently, a diffraction-limited map done across a $50 \times 50$ $\mu \mathrm{m}^{2}$ region, created by raster-scanning focal spots at a typical integration time of 1 $s$, would take half a day. As a result, many previous studies only collected a single spectrum from a few spots, despite the fact that large-area scanning and volumetric scanning are highly desired for accurate and systematic studies of material properties. In principle, the Raman signal intensity per pixel is proportional to the light dose, which is the product of the laser power and measurement time per pixel (and therefore inversely proportional to the scanning rate). A better signal-to-noise ratio (SNR) could be achieved by increasing the laser power. However, the laser power cannot generally be increased without considering potential light-induced damage to the sample. For graphene, structural changes are observed in response to laser irradiation in the $\mathrm{mW}$ range ${ }^{[13,31]}$. To mitigate this problem, an electron-multiplying charge-coupled device (EMCCD), available in many modern Raman instruments, has been used to amplify the SNR such that a higher scanning speed of several tens of milliseconds per spectrum can be delivered at $1 \mathrm{~mW}$ laser power ${ }^{[30,32]}$. Higher speed, however, is still needed for large scale volumetric scanning. Recently, widefield Raman imaging ${ }^{[33]}$ was introduced to map large area graphene sheets in a few seconds. However, this technique requires additional hardware components and is typically limited to one or few frequency bands, undermining its performance for quantitative characterization of many physico-chemical properties such as defect density. The applicability in depth-resolved volumetric ${ }_{3} \mathrm{D}$ scanning also remains challenging. For GO, the low throughput problem is even more severe due to the requirement of an extremely low laser dose to suppress reduction. A recent study 
suggested that even a laser dose of $48 \mu \mathrm{J}$, i.e. $48 \mu \mathrm{W}$ in $1 \mathrm{~s}$, is still too high to prevent sample damage ${ }^{[34]}$.

In recent decades, numerous studies demonstrated that imaging-related challenges can often be addressed with post-data-analysis using mathematical algorithms ${ }^{[35-37]}$. Principal component analysis (PCA), an algorithm that is widely used in signal processing and machine learning to find common features in the dataset $^{[38]}$, has been applied to improve the SNR of hyperspectral datasets ${ }^{[39,40]}$. The idea is that, by analyzing the variance between spectra within the whole dataset, PCA can distinguish signal features from noise features and thereby allowing a reconstruction of the dataset with predominantly signal features. The performance of PCAguided denoising generally increases with the size of the dataset, because larger datasets enable a more thorough extraction of the signal features. Therefore, it is ideal for large scale hyperspectral analysis.

In this work, we introduce an algorithm-improved confocal Raman microscopy (aiCRM), combining PCA and EMCCD, to image 2D materials. Briefly, we first collect spectra with an EMCCD at high scanning speed and low laser power. The combination of short measurement time per pixel and low laser power results in "noisy" spectra with a SNR below one. Then we recover the faint signal, invisible in the noise, with the help of PCA. With this technique, Raman mapping of GO can be performed with an extremely low laser power of $5 \mu \mathrm{W}$, close to hardware limitation, together with short integration times of $10 \mathrm{~ms}$ per spectrum. Meanwhile, we demonstrate that such low laser dose per spectrum can effectively prevent GO reduction. Graphene can be mapped at a hardware-limited scanning rate of $1 \mathrm{~ms}$ per spectrum at $1 \mathrm{~mW}$ laser power. For graphene and GO, the power averaged scanning rate (scanning rate divided by power for fair comparison) in our work is more than 1 order of magnitude faster than state-of-the-art works. Finally, we demonstrate that ai-CRM can be extended to fast imaging of other $2 \mathrm{D}$ materials including $\mathrm{MoS}_{2}, \mathrm{WS}_{2}$ and $\mathrm{BN}$, and fast volumetric imaging of composite materials. 


\subsection{Experimental section}

\section{Materials}

A GO suspension ( $2 \mathrm{mg} \mathrm{mL}^{-1}, 22 \mu \mathrm{m}$ mean diameter flakes, Sigma Aldrich) was diluted with $1 \mathrm{~mL}$ of deionized water (Millipore) and was deposited on plasmacleaned $300 \mathrm{~nm}-\mathrm{SiO}_{2} / \mathrm{Si}$ wafers by drop casting. CVD-grown single layer graphene on glass and $300 \mathrm{~nm}-\mathrm{SiO}_{2} / \mathrm{Si}$ wafer were purchased (Graphene supermarket, USA) and used directly for Raman imaging. Standard laboratory grade glass coverslips and Raman grade calcium fluoride (Crystran Ltd., UK) were used as alternative substrates. $\mathrm{MoS}_{2}, \mathrm{WS}_{2}$ and $\mathrm{BN}$ were mechanically exfoliated from their bulk crystals (HQ Graphene, the Netherlands) with Scotch tapes. $\mathrm{MoS}_{2}$ and $\mathrm{WS}_{2}$ were directly exfoliated on $300 \mathrm{~nm}-\mathrm{SiO}_{2} / \mathrm{Si}$ wafers. $\mathrm{BN}$ was exfoliated on a polydimethylsiloxane (PDMS) film and then transferred to a $300 \mathrm{~nm}-\mathrm{SiO}_{2} / \mathrm{Si}$ wafer.

\section{Raman measurements}

Raman measurements were carried out using a WiTec alpha 300R Raman microscope connected to a $532 \mathrm{~nm}$ laser. A $600 \mathrm{~g} / \mathrm{mm}$ grating was used, which provided a spectral resolution of around $2.3 \mathrm{~cm}^{-1}$. An EMCCD camera $(1600 \times 200$ pixels, 16 $\mu \mathrm{m}$ pixel size, Andor Newton) was used for detection of the scattered photons. The EMCCD gain was set at a numerical value of 250 . Pre-amplifier gain value was set to 1. For high spatial as well as depth resolution, a 100x objective (Zeiss EC "EpiplanNeofluar” DIC, Numerical Aperture $(\mathrm{NA})=0.9$ ) was chosen. The laser power at the sample was measured using an optical power meter (ThorLabs). Raman maps are made by integrating the area under the band of interest for every pixel : from $1550 \mathrm{~cm}^{-1}$ to $1750 \mathrm{~cm}^{-1}$ ( $\mathrm{G}$ band) for GO and graphene, $2600 \mathrm{~cm}^{-1}-2800 \mathrm{~cm}^{-1}$ for the $\mathrm{G}^{\prime}$ peak of graphene, $253 \mathrm{~cm}^{-1}-400 \mathrm{~cm}^{-1}$ for $\mathrm{WS}_{2}, 1330 \mathrm{~cm}^{-1}-1390 \mathrm{~cm}^{-1}$ for $\mathrm{BN}$, $350 \mathrm{~cm}^{-1}-427 \mathrm{~cm}^{-1}$ for $\mathrm{MoS}_{2}$ and $2800 \mathrm{~cm}^{-1}-3000 \mathrm{~cm}^{-1}$ for PAA. 


\section{Denoising}

Denoising was performed on MATLAB (version R2017b) with home-written codes. Typically, denoising 10000 spectra only takes around 10-20 seconds with a normal office computer. Cosmic rays are removed prior to PCA. More information about the denoising technique can be found in chapter 2 of the thesis.

\subsection{Results and Discussion}

\subsubsection{Fast mapping of GO}

We first demonstrate our protocol (Figure 3.1) with a typical Raman mapping of GO nano-sheets. Aqueous GO dispersion $(2 \mathrm{mg} / \mathrm{mL})$ was drop-cast on a plasma cleaned $300 \mathrm{~nm}-\mathrm{SiO}_{2} / \mathrm{Si}$ wafer. Confocal Raman mapping (Figure 3.1(a)) was conducted by raster scanning over $25 \times 25 \mu \mathrm{m}^{2}$ with a step size of $0.25 \mu \mathrm{m}$, using a laser with a wavelength of $\lambda=532 \mathrm{~nm}$. To avoid sample damage, the laser power was kept at a power of $5 \mu \mathrm{W}$ underneath the objective. A 1oox objective with a numerical aperture (NA) of 0.9 was used, and the estimated laser spot size, $d$ is $1.22 \lambda / \mathrm{NA}=$ $0.72 \mu \mathrm{m}$, where $\lambda$ is the laser wavelength, corresponding to a power density of 12.3 $\mu \mathrm{W} / \mu \mathrm{m}^{2}$. The diffraction limited resolution is $\mathrm{d} / 2=0.36 \mu \mathrm{m}$. An EMCCD was used to collect the spectra with an integration time of $20 \mathrm{~ms}$ for each spectrum. After the scan, Raman spectra from all pixels are assembled into a data matrix where each row contains a spectrum (Figure 3.1(b)). The Raman spectrum from 41-3692 $\mathrm{cm}^{-1}$ was selected for analysis. The collected spectra are extremely noisy, as shown by the representative spectrum in Figure 3.1 (c). The Raman signal of GO cannot be identified. Subsequently, we apply PCA to decompose the dataset into its principal components (PCs. Note that these components are not real spectra, but spectra of the variance in the dataset) whose number is equal to that of the number of wavenumber steps ( 1571 in this case). The PCs are ranked according to their percentage of total variance described, which represents the importance to the dataset ${ }^{[38]}$. Since the noise 


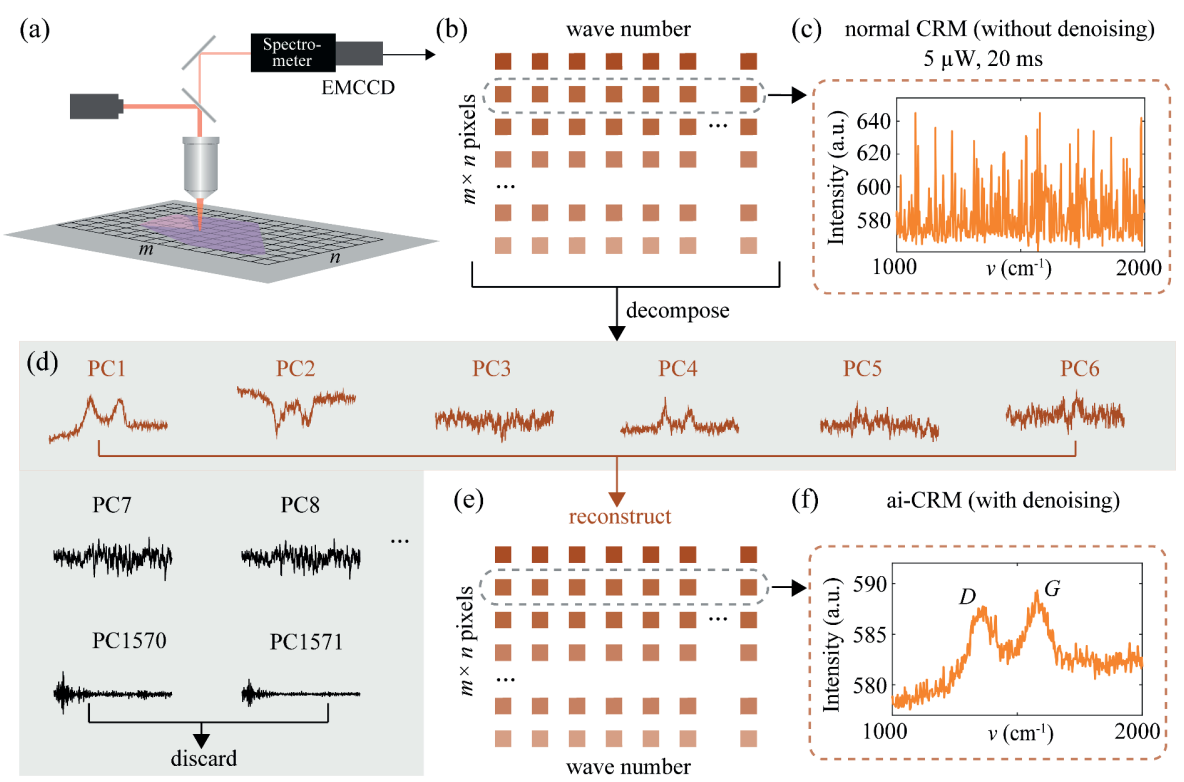

Figure 3.1: Schematic illustrating the ai-CRM procedure. (a) Raw dataset containing $m x n$ pixels is collected using an EMCCD, and (b) assembled into a matrix in which each row contains a spectrum of a pixel and each column represents a wavenumber. (c) A representative noisy spectrum from a dataset recorded on a GO sample. (d-e) The principal components (PC) from a principal component analysis (PCA) of the measured dataset. PCA effectively decomposes the dataset into a low dimensional space (here the first 6 PCs) containing mostly signal and a high dimensional space (PCs 7 to end) that contain mostly noise. PCs carry signal information and are used to reconstruct the dataset (e), whereas the rest of the PCs are set to "zero" in the reconstruction. $(f)$ Reconstructed spectrum of the same pixel as in (c) with dramatically improved SNR clearly showing the D and G band of GO. 
in the spectra is random, while signals are a recurring feature in a fraction of the pixels in the dataset, the PCs containing signals will contribute much more to the variance than those containing mostly noise (see Figure 2.2, Chapter 2). Typically, only the first few PCs contain obvious signal information. We use a Scree test ${ }^{[41]}$ to determine the number of PCs (here: 6) that need to be retained for further analysis (Figure 2.2(b), Chapter 2). Relatively clear band-like features are observed in these first PCs (Figure 3.1 (d)), but not in the subsequent ones, which contain mostly noise. Using the first 6 PCs to reconstruct the dataset and rejecting the 1565 "noise-dominated" ones out of the total of 1571 PCs, we obtain Raman spectra with dramatically improved SNR that clearly display the distinct D and G band (Figure 3.1(f)). The criteria to choose the number of PCs is loose. A few more or less than that suggested by the Scree test is typically acceptable (Figure 2.3, Chapter 2). The spectra in Figure 3.1(c) and Figure 3.1(f) correspond to the same pixel in the image.

To demonstrate the efficiency of our method for mapping, we imaged the GO nanosheet with an atomic force microscope (AFM) (Figure 3.2(a)), and compared it with the Raman images (Figure 3.2(b) and Figure 3.2(c)) created by integrating the G band, with and without ai-CRM, respectively. For clarity, peak intensities will be denoted by I and integrated band area intensities will be denoted by A. The two images show the same region of interest. The distribution of single layer $(1 \mathrm{~L})$, double layer $(2 \mathrm{~L})$, and triple layer $(3 \mathrm{~L}) \mathrm{GO}$ can be clearly resolved after applying ai-CRM (Figure 3.2(c)). The integrated $G$ band intensity $(A(G))$ and AFM height profile on a selected line through the same positions (green line in Figure 3.2(b) and blue line in Figure 3.2(a)), shown in Figure 3.2(d) demonstrates an excellent correspondence. In contrast, with normal CRM (i.e., without denoising), the GO nano-sheet can only be vaguely observed in the Raman intensity profile of Figure $3.2(\mathrm{~b})$ and the number of layers cannot be distinguished, even when the integration time per pixel is increased to $500 \mathrm{~ms}$ (Figure 3.2(e)). Using ai-CRM, we can reduce the integration time even further down to 1 oms per pixel: the GO layers in the nano-sheet (Figure 

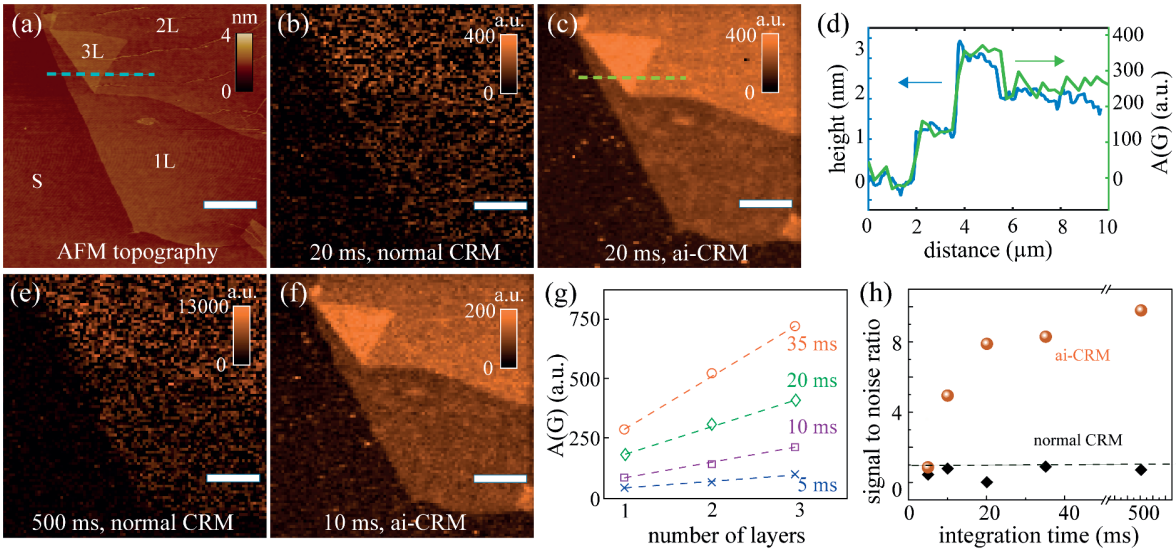

Figure 3.2: Fast Raman mapping of GO ( $G$ band) with ai-CRM (a) AFM image of the probed GO sheets, showing the distribution of single layer $(1 \mathrm{~L})$, two-layer $(2 L)$, and threelayer ( $3 \mathrm{~L}$ ) on a $300 \mathrm{~nm}-\mathrm{SiO}_{2} / \mathrm{Si}$ substrate $(\mathrm{S})$. (b) Normal CRM raw image prior to denoising. At $20 \mathrm{~ms}$ integration time, the configuration of $G O$ can only be vaguely discerned. (c) After denoising with ai-CRM, the configuration of the GO sheets can be clearly resolved, and (d) matches with the AFM image in the pixel scale, as demonstrated by the largely overlapped AFM height profile and Raman intensity profile crossing the same positions (blue line in (a) and green line in (c)). (e) Normal CRM map with a high integration time of $500 \mathrm{~ms}$. $(\mathrm{f})$ ai-CRM image with integration time lowered to $10 \mathrm{~ms}$, depicting the fastest mapping possible with our method without compromising on the layer identification. (g) Variation of the intensity with layer number for 4 different integration times, showing possibility for fast quantification with ai-CRM. The dashed lines are guides to the eye. (h) Variation of SNR with integration time, depicting the amplification of the SNR achieved with ai-CRM as compared to normal CRM data. Scale bars are $5 \mu \mathrm{m}$ and the resolution of the Raman maps are $100 \times 100$ pixels. 

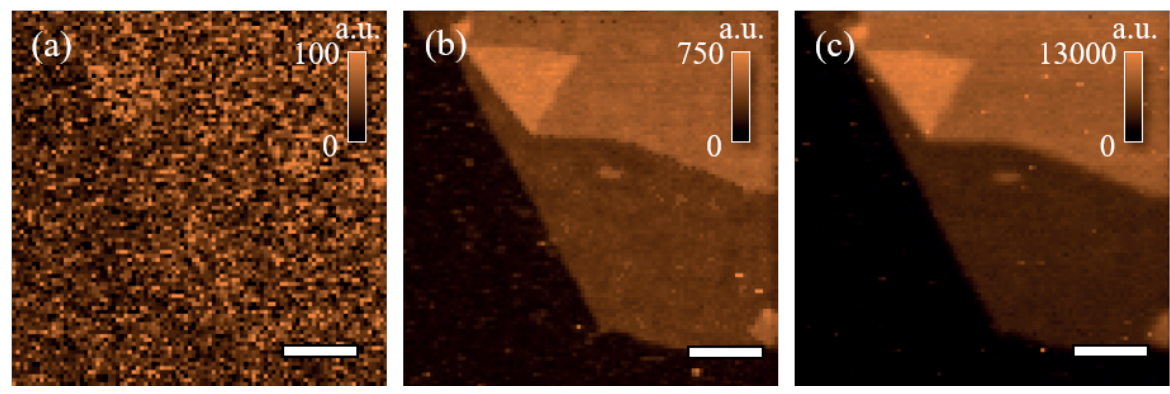

Figure 3.3: ai-CRM maps of GO nanosheets corresponding to Figure 3.2. Denoised Raman maps at an integration time of (a) $5 \mathrm{~ms},(b) 35 \mathrm{~ms}$ and (c) $500 \mathrm{~ms}$. The scale bars correspond to $5 \mu \mathrm{m}$.

3.2(f)) are still clearly visible, confirming the efficiency of our method to facilitate fast mapping.

The ai-CRM data also allows quantitative analysis of the properties of GO. For example, the results (Figure $3.2(\mathrm{~g})$ ) show that the integral intensity of G band is linearly related to the number of layers and the integration time, which ranges here from 5 to $35 \mathrm{~ms}$. This stems from the fact that the Raman intensity linearly scales with the volume of material within the confocal laser spot. This also implies the $2 \mathrm{~L}$ and ${ }_{3} \mathrm{~L}$ GO here are weakly coupled, suggesting that ai-CRM could be used for fast counting of layer numbers in the current system. To quantify the SNR enhancement by our method, the average and the standard deviation of 400 randomly selected spectra were calculated for both normal CRM and ai-CRM datasets for each integration time. The SNR was then determined from the following equation:

$$
\mathrm{SNR}=\mathrm{I}(\mathrm{G}) / \Delta \mathrm{I}_{\text {noise }}
$$

where $\mathrm{I}(\mathrm{G})$ is the peak intensity of $\mathrm{G}$ band in the averaged spectrum and $\Delta \mathrm{I}_{\text {noise }}$ is the standard variation calculated from the same spectrum at a wavenumber region 
(2000-2200 $\left.\mathrm{cm}^{-1}\right)$ where no bands of these materials occurs. Figure $3.2(\mathrm{~h})$ shows that with CRM, the SNR is lower than 1 for all integration time from 5 to $500 \mathrm{~ms}$, leading to noisy images (e.g. Figure 3.2(b) and Figure 3.2(e)). Note that a SNR below 1 means that signal cannot be clearly distinguished from noise, and therefore the values of SNR are only a rough estimation. With ai-CRM, however, the SNR increases dramatically and progressively increases with integration time. Importantly, even the SNR for $10 \mathrm{~ms}$ integration is much higher than for any of the integration times without denoising. This suggests that our method can increase the scanning speed by more than 50 times from $500 \mathrm{~ms}$ to $10 \mathrm{~ms}$ at an improved SNR. For the ai-CRM Raman maps at other integration times, refer Figure 3.3. In addition, a literature review suggests that the power averaged scanning rate (scanning rate divided by power for a more fair comparison since Raman signal intensity is proportional to the laser dosage) in this work is 2-7 orders of magnitude higher than previous works (Figure 3.8(a)).

\subsubsection{Non-invasive mapping of GO}

For GO, reliable Raman characterization has been a major challenge due to laserinduced sample damage ${ }^{[34,42]}$. To mitigate this problem, the laser dose needs to be reduced as much as possible ${ }^{[11,34,42]}$. Many previous works used a mW scale power to characterize $\mathrm{GO}^{[43-47]}$. A recent study ${ }^{[34]}$ suggested that laser-induced reduction of GO cannot be prevented even when the laser intensity is down to a dose of $8 \times 10^{7} \mathrm{~J} / \mathrm{m}^{2}$, which corresponds to $48 \mu \mathrm{W}$ during $1 \mathrm{~s}$ in the confocal spot. Such laser intensity is already a few orders of magnitude lower compared to preceding studies ${ }^{[46,47]}$. Further reduction of the laser power, however, resulted in Raman spectra with insufficient SNR. However, the results in Figure 3.2 suggest that ai-CRM enables characterization of GO at two orders of magnitude lower intensity (tens of ms with a laser power of $5 \mu \mathrm{W}$ ).

For a comparative study with ai-CRM, we selected two levels of laser power, $4 \mu \mathrm{W}$ 

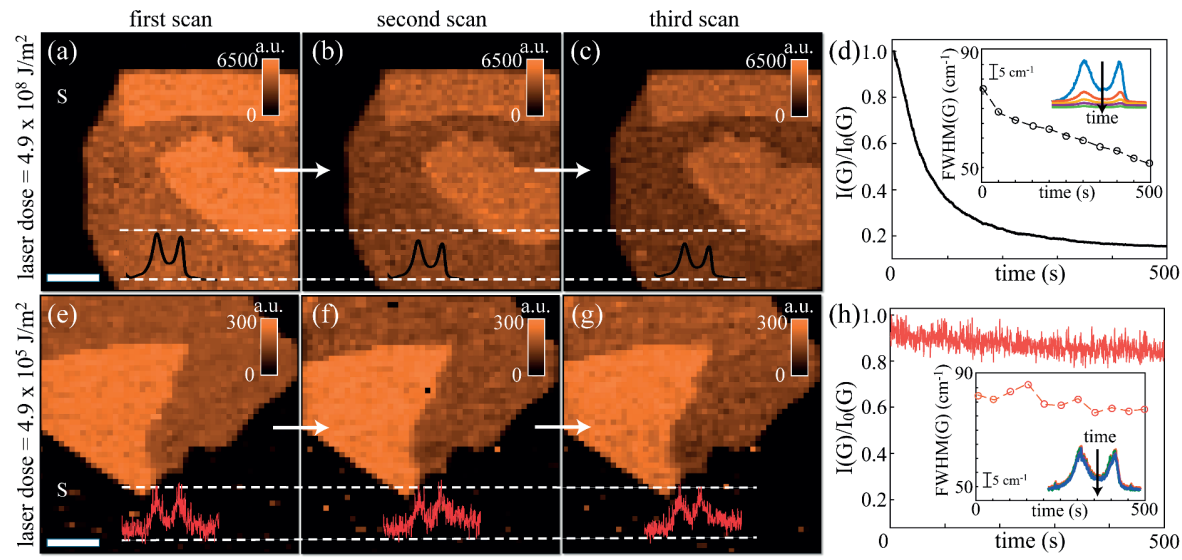

Figure 3.4: Reliable Raman mapping of GO at minimum laser power with ai-CRM. (a), (b) and (c) Raman maps of $G O(A(G))$ done at the same location, one after the other using a CCD, with a laser power of $4 \mathrm{~mW}$. (e), $(f)$ and $(g)$ Raman images of $G O$ done using ai-CRM, at 10oox reduced laser power ( $4 \mu \mathrm{W})$ showing minor loss of contrast and integrated intensity. $(d)$ and $(h)$ Variation of the normalized intensity of the G-peak of $G O$, obtained from a single pixel time series data for $(d) 4 \mathrm{~mW}$ and for $(h) 4 \mu \mathrm{W}$. The insets show the variation of the spectra as well as the FWHM of the G peak. All data are recorded at $50 \mathrm{~ms}$ integration time per pixel. All scale bars correspond to $5 \mu \mathrm{m}$ and all Raman maps have a resolution of $50 x$ so pixels. 
and $4 \mathrm{~mW}$ (Figure 3.4), to determine the influence of laser power on the Raman signal of GO. Note that for the $4 \mathrm{~mW}$ case, normal CCD instead of EMCCD was used, because at such high laser power in the EMCCD mode, the intensity of silicon peak exceeds upper detection limit. This has no effect on the sample damage analysis. Figures 3.4(a),(b) and (c) show the Raman images obtained at $4 \mathrm{~mW}$ of one nano-sheet of GO and Figures 3.4(e),(f) and (g) show the Raman images at $4 \mu \mathrm{W}$ of another nano-sheet of GO. All images in the same series are presented against the same color scale. Each pixel was measured for $50 \mathrm{~ms}$ in both situations. The loss in $\mathrm{A}(\mathrm{G})$ in going from Figure 3.4(a) to Figure 3.4(c) is apparent from the reduction of contrast at $4 \mathrm{~mW}\left(4.9 \times 10^{8} \mathrm{~J} / \mathrm{m}^{2}\right.$ intensity), which indicates the gradual increase in sample damage upon repeated illumination. The decrease in Raman intensity is also seen in the average spectrum for each image (insets in Figures 3.4(a)-(c)). Laser-induced reduction (to reduced graphene oxide, rGO) during Raman imaging has been observed on all GO nano-sheets (see e.g. Figure 3.5). This was further confirmed by measuring the Raman spectrum of a single spot during $500 \mathrm{~s}$ of continuous laser irradiation of $4 \mathrm{~mW}$ with a spectrum obtained every $50 \mathrm{~ms}$. The Raman intensity decreased rapidly by 70 percent in the first $100 \mathrm{~s}$ and gradually decreased further to a weak plateau value of residual scattering (Figure 3.4(d)). In addition, the full width half maximum (FWHM) of the G band decreased monotonically with time (inset Figure 3.4(d)), and the $\mathrm{I}(\mathrm{D}) / \mathrm{I}(\mathrm{G})$ ratio changes with time (Figure 3.5 $(\mathrm{a})$ ), which is a typical consequence of the reduction of $\mathrm{GO}^{[34]}$. In contrast, the intensity loss is substantially reduced when illuminated with $4 \mu \mathrm{W}$ of laser irradiation $\left(4.9 \times 10^{5} \mathrm{~J} / \mathrm{m}^{2}\right.$ intensity) for $500 \mathrm{~s}$ with $50 \mathrm{~ms}$ per spectrum (Figure $3.4(\mathrm{~h})$ and the insets, respectively). In addition, scanning did not cause any obvious optical changes to the bright-field image of the sample (Figure 3.5(b)). After 500 s exposure at the same pixel, the change in $\mathrm{I}(\mathrm{G})$ is less than 10 percent. The change in FWHM is also negligible as it is close to the wavenumber resolution of the instrument $\left(\approx 2 \mathrm{~cm}^{-1}\right)$. These results clearly confirm the efficiency of ai-CRM to suppress sample damage after reduction of the experimental laser intensity. 
(a)

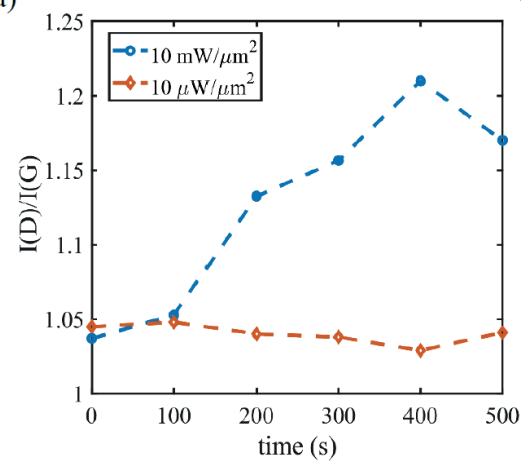

(b)

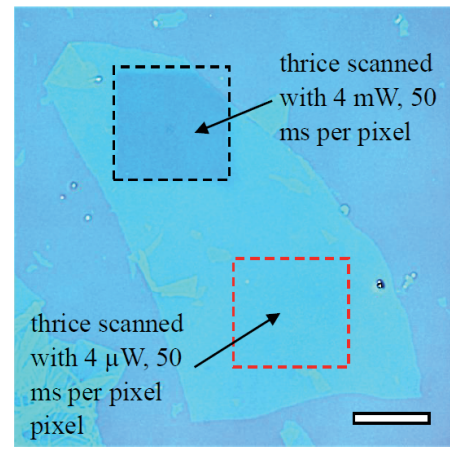

Figure 3.5: Preventing the reduction of $G O$. (a) Variation of the $I(D) / I(G)$ ratio with time corresponding to Figures $3.4(d)$ and $(h)$. Significantly greater change in ratio is observed for the $4 \mathrm{~mW}$ case $\left(10 \mathrm{~mW} / \mu \mathrm{m}^{2}\right)$ as compared to the $4 \mu \mathrm{W}$ case $\left(10 \mu \mathrm{W} / \mu \mathrm{m}^{2}\right)$, hinting at laser induced reduction. (b) Optical image showing a change in reflection contrast of a GO sheet when mapped thrice with $4 \mathrm{~mW}$ laser power, whereas no change is observed when scanned with a low laser power of $4 \mu \mathrm{W}$. This shows the non-invasive nature of the protocol. Scale bar corresponds to $20 \mu \mathrm{m}$. 


\subsubsection{Fast mapping/quality analysis of graphene}

Our method can be also applied for fast Raman mapping of graphene. To demonstrate this, we made a scratch on a single layer of graphene grown by chemical vapor deposition (CVD) on a $300 \mathrm{~nm}-\mathrm{SiO}_{2} / \mathrm{Si}$ wafer. Then we performed a Raman scan across $25 \times 25 \mu \mathrm{m}^{2}$ region with a step size of $0.25 \mu \mathrm{m}$ at $1 \mathrm{~mW}$ laser power, which was previously confirmed to be non-destructive for graphene ${ }^{[32]}$. Similar to the results of GO, significant SNR improvement was observed, as shown by the Raman images of the integrated $\mathrm{G}^{\prime}$ band intensity generated at $5 \mathrm{~ms}$ integration time before and after applying ai-CRM (Figure 3.6(a) and (b)). Before denoising, the D, G, and $\mathrm{G}^{\prime}$ bands can hardly be seen (bottom spectrum in Figure 3.6(a)). Although different layer numbers can still be distinguished, an accurate quantitative analysis is hardly possible. After denoising, a weak D band, a sharp $\mathrm{G}$ band, together with a sharp and strong $\mathrm{G}^{\prime}$ band are clearly resolved (bottom spectrum in Figure 3.6(b)), indicating the high quality of the graphene sample. The ai-CRM Raman images at other integration times (1 ms, $10 \mathrm{~ms}$ and $35 \mathrm{~ms}$ ) are shown in Figure 3.7. The Raman intensity $\left(\mathrm{I}\left(\mathrm{G}^{\prime}\right), \mathrm{I}(\mathrm{G})\right.$ and $\mathrm{I}(\mathrm{D})$ ) of the double layer graphene (labeled as $2 \mathrm{x} 1 \mathrm{~L}$ in Figure 3.6(a)) is roughly twice that of the single layer graphene, confirming that the double layer is composed of two weakly coupled single layers.

Importantly, the ai-CRM data enables quantitative analysis of the sample quality. The distance between defects, $\mathrm{L}_{\mathrm{D}}$ can be estimated from the ratio of $\mathrm{I}(\mathrm{G})$ to $\mathrm{I}(\mathrm{D})^{[17]}$ :

$$
\mathrm{L}_{\mathrm{D}}^{2}\left(\mathrm{~nm}^{2}\right)=1.8 \times 10^{-9} \lambda^{4} \mathrm{I}(\mathrm{G}) / \mathrm{I}(\mathrm{D})
$$

where $\lambda$ is in nanometers. The distribution of $L_{D}$ is then plotted in Figure 3.6(c). It is observed that the graphene sample has a relatively uniform $L_{D}$ of $\approx_{20} \mathrm{~nm}$ across the scanned area, again confirming its high quality. Note that regions having $\mathrm{L}_{\mathrm{D}}<10 \mathrm{~nm}$ are not plotted (scale bar in Figure 3.6(c) starts from $10 \mathrm{~nm}$ ) because equation 3.2 
(a) $5 \mathrm{~ms}$, normal CRM

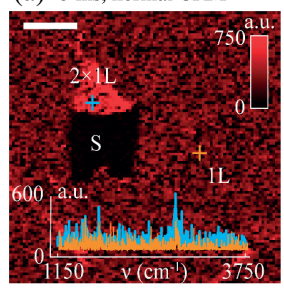

(b) $5 \mathrm{~ms}$, ai-CRM

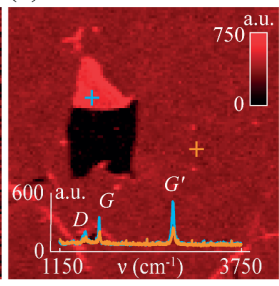

(c) defect distance

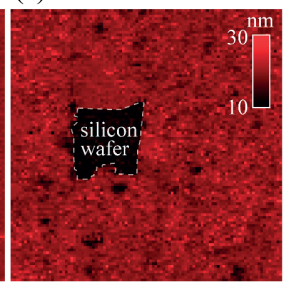

(d)

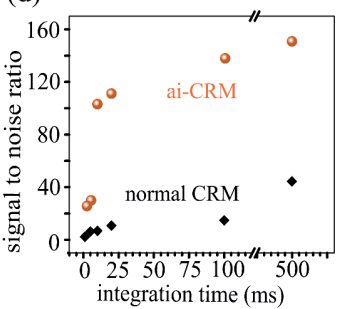

Figure 3.6: Fast Raman mapping and quality analysis of graphene with ai-CRM (a) Normal CRM map of CVD grown graphene on $300 \mathrm{~nm}-\mathrm{SiO}_{2} / \mathrm{Si}$ wafer at $1 \mathrm{~mW}$ laser power and $5 \mathrm{~ms}$ integration time. The inset shows the spectra corresponding to the markers on $a$ double single layer (blue) and single layer (yellow) (b) ai-CRM map of the same region. The spectra show the evolution of the $D, G$ and $G^{\prime}$ peaks after denoising, useful for quantitative analysis. (c) Color-coded defect density map, derived from the peak intensity ratio of $D$ and $G$ band (Equation 3.2), of the same region depicting that the average distance between defects is around $20 \mathrm{~nm}$, showing the high quality of the CVD grown graphene. (d) Variation of the SNR of the G' peak for various integration times, illustrating the amplification of the SNR with ai-CRM.
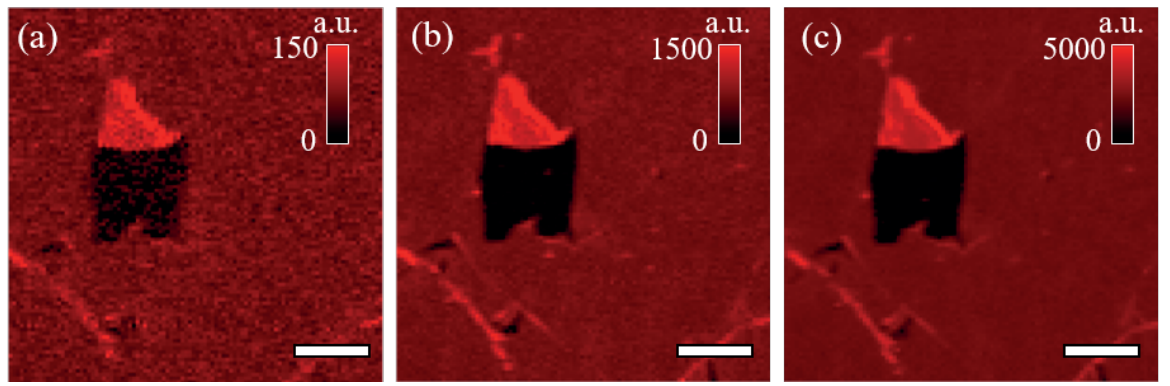

Figure 3.7: ai-CRM maps of graphene corresponding to Figure 3.6. Denoised Raman maps at an integration time of (a) $1 \mathrm{~ms},(b) 10 \mathrm{~ms}$ and (c) $35 \mathrm{~ms}$. The scale bars correspond to $5 \mu \mathrm{m}$. 
is not valid in this case. It should be noted that the reduction of $\mathrm{GO}$ also changes the defect density, thereby changing the I(D)/I(G) ratio, as shown in Figure 3.5 (a). The above results suggest the potential of ai-CRM for spatially resolved and fast characterization of the defect density, which should help in the quality control of graphene fabrication. Furthermore, to quantify the improvement in scanning rate, we scanned the same sample using different integration times from $1 \mathrm{~ms}$ to $500 \mathrm{~ms}$, and calculated the SNR for all datasets before and after applying ai-CRM. Results in Figure 3.6(d) show that the SNR at 500 ms without denoising is comparable to the SNR at $5 \mathrm{~ms}$ with denoising, and is much lower than that at $10 \mathrm{~ms}$ with denoising. Similar to the results for GO, this demonstrates again that an increase of more than 50 times in scanning rate can be achieved when ai-CRM is applied. Compared to literature, the power averaged scanning rate here is around two orders of magnitude higher than state-of-the-art works (Figure 3.8(b)).

\subsubsection{Fast mapping of other $2 \mathrm{D}$ materials}

ai-CRM was also tested on mechanically exfoliated $\mathrm{MoS}_{2}, \mathrm{WS}_{2}$ and $\mathrm{BN}$ nanosheets. The optical and AFM characterization of the three materials are shown in Figure 3.9. $\mathrm{MoS}_{2}$ and $\mathrm{WS}_{2}$ can be imaged at $50 \mathrm{~ms}$ integration time and under a laser power of 20 $\mu \mathrm{W}$ (Figure 3.10(a) and (b)). BN, having a smaller Raman scattering cross section, can be imaged at $50 \mathrm{~ms}$ integration time under a $500 \mu \mathrm{W}$ laser (Figure $3.10(\mathrm{c})$ ). Note that the ai-CRM maps and spectra of these materials generated at $50 \mathrm{~ms}$ integration time have a similar or better quality than the normal CRM maps and spectra at 500 ms integration time (compare with Figure 3.11), suggesting that ai-CRM increases the scanning speed by at least 10 times while still maintaining a higher SNR.

\subsubsection{Fast volumetric Raman imaging}

Volumetric imaging is another advantage of CRM, which can help, for e.g., to assess the properties of graphenic materials inside a composite or a device. However, such 

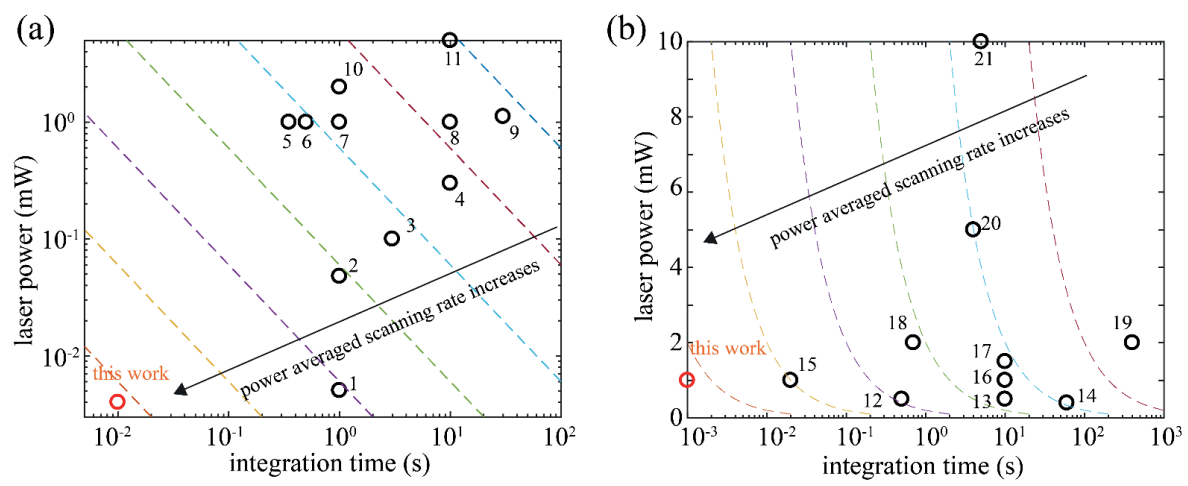

Figure 3.8: Comparison of our imaging conditions with previous literature. Laser power vs. integration time used for (a) graphene oxide - ${ }^{[48]}, 2^{[34]}, 3^{[49]}, 4^{[50]}, 5^{[51]}, 6^{[46]}, 7^{[47]}$, $8^{[52]}, 9^{[53]}, 10^{[54]}$ and $11^{[55]}$, and (b) graphene - $12^{[30]}, 13^{[56]}, 14^{[57]}, 15^{[32]}, 16^{[24]}$, $17^{[29]}, 18^{[25]}, 19^{[58]}, 20^{[26]}$ and $21^{[59]}$. Note that this is only a rough comparison because different studies use different instruments, objectives and operation conditions, which all affect the scanning rate. The gap between neighbouring dashed lines represent one order of magnitude difference. Note that the $y$-axis is in logarithmic scale in (a) and in linear scale in (b). 

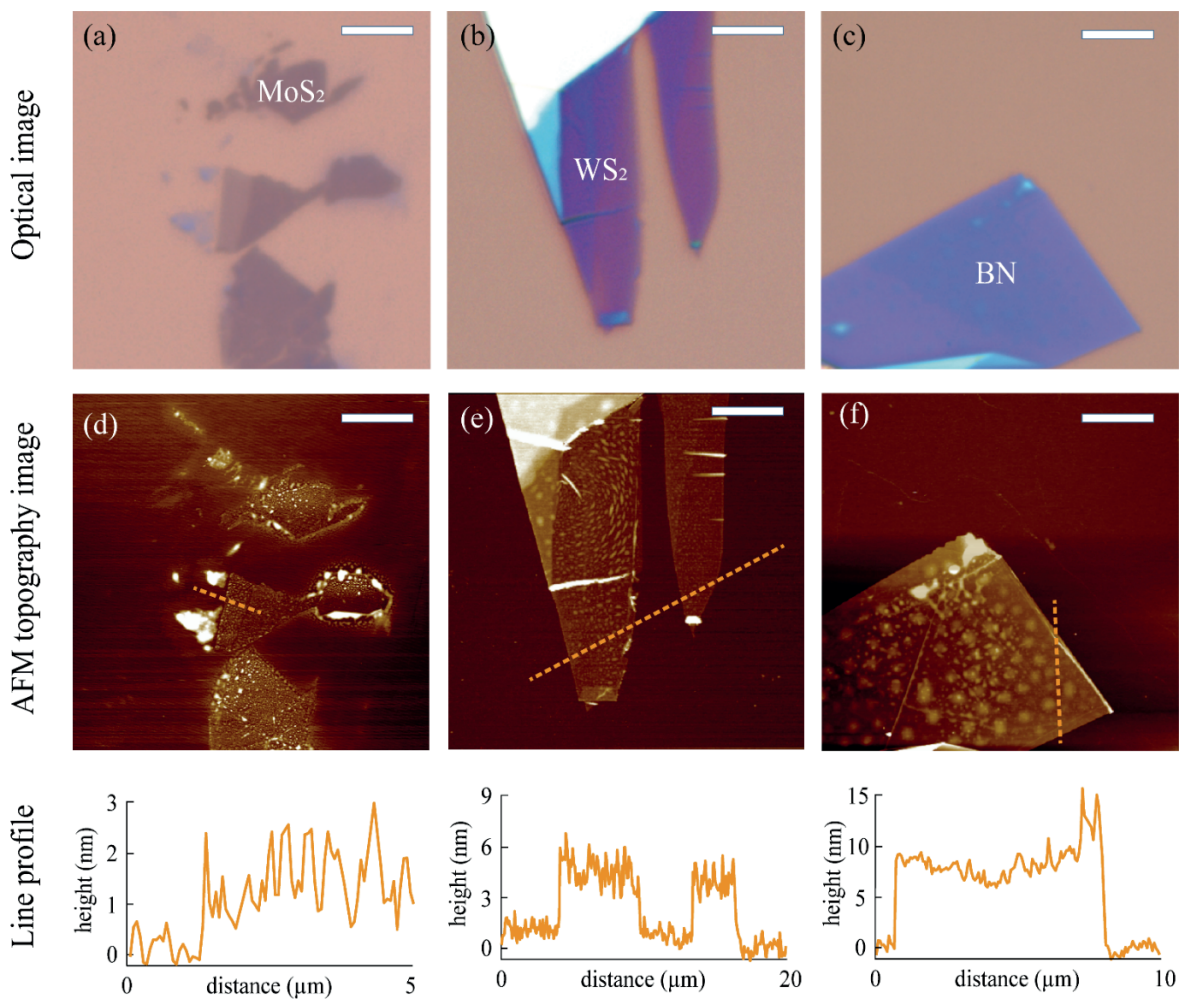

Figure 3.9: Optical and AFM characterization of $\mathrm{MoS}_{2}, W S_{2}$ and BN. (a), (b) and (c) show the optical images of the mechanically exfoliated $\mathrm{MoS}_{2}, \mathrm{WS}_{2}$ and $\mathrm{BN}$ respectively. (d), (e) and ( $f$ ) show the AFM topography images with the corresponding line profiles (below) of the three materials respectively. The scale bars correspond to $5 \mu \mathrm{m}$. 
(a) $50 \mathrm{~ms}, 20 \mu \mathrm{W}$, ai-CRM

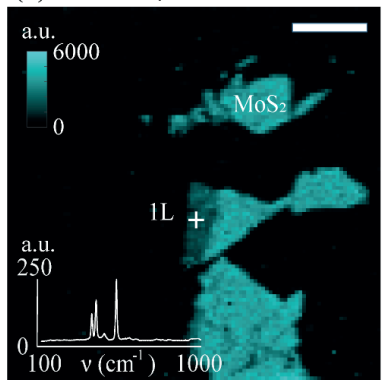

(b) $50 \mathrm{~ms}, 20 \mu \mathrm{W}$, ai-CRM

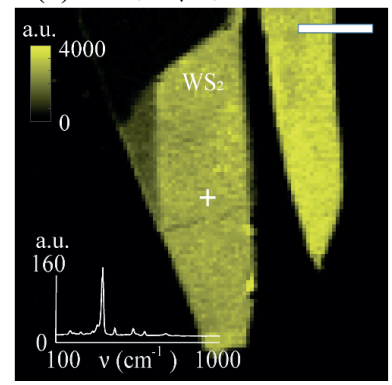

(c) $50 \mathrm{~ms}, 500 \mu \mathrm{W}$, ai-CRM

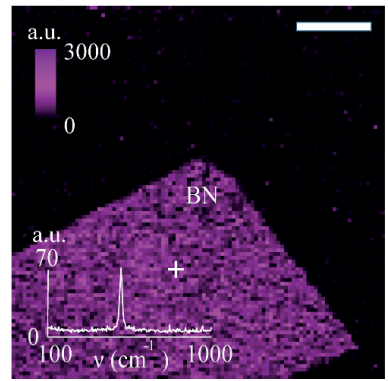

Figure 3.10: Fast mapping of mechanically exfoliated $\mathrm{MoS}_{2}, \mathrm{WS}_{2}$ and $\mathrm{BN}$ with ai-CRM (a) Raman mapping of $\mathrm{MoS}_{2}$ (1L at cross-hair) (b) $\mathrm{WS}_{2}\left(\approx_{5} \mathrm{~nm}\right.$ at cross-hair) and (c) $B N(\approx 9 n m$ at cross-hair) with denoising. The denoised spectra are shown in the inset at the location of the cross-hair. The laser power and integration times are mentioned in the figures. Resolution of the Raman maps are $100 \times 100$ pixels. The scale bars correspond to $5 \mu \mathrm{m}$.

(a) $500 \mathrm{~ms}, 20 \mu \mathrm{W}$, normal CRM

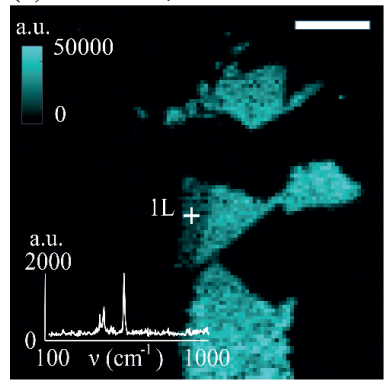

(b) $500 \mathrm{~ms}, 20 \mu \mathrm{W}$, normal CRM

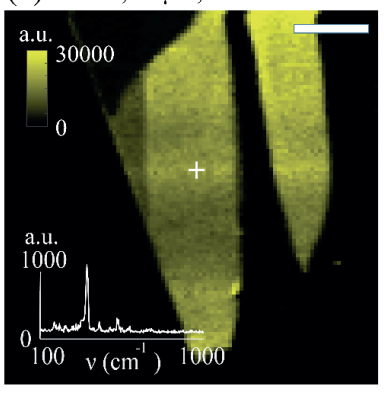

(c) $500 \mathrm{~ms}, 500 \mu \mathrm{W}$, normal CRM

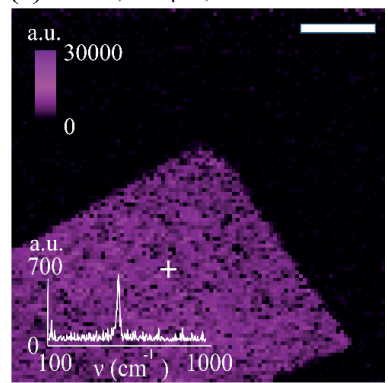

Figure 3.11: Normal CRM images of $\mathrm{MoS}_{2}, \mathrm{WS}_{2}$ and BN corresponding to Figure 3.10. (a) Raman mapping of $\mathrm{MoS}_{2}$ (b) WS $\mathrm{W}_{2}$ and (c) BN at $500 \mathrm{~ms}$ integration time per pixel. The raw spectra are shown in the inset at the location of the cross-hair. The laser power and integration times are mentioned in the figures. Resolution of the Raman maps are 100 $x 100$ pixels. The scale bars correspond to $5 \mu \mathrm{m}$. 
a potential advantage has not been exploited in previous studies due to the long measurement times required. Since one $2 \mathrm{D}$ Raman image across tens of micrometers at diffraction limited spatial resolution could take half a day, a volumetric Raman image created by stacking multiple $2 \mathrm{D}$ images would take several days. With our method, it is now possible to reduce the time to around 10 minutes. To demonstrate this, a GO dispersion was mixed with aqueous polyacrylic acid (PAA), and the mixture was cured overnight in an oven at $80^{\circ} \mathrm{C}$. After curing, the $\mathrm{GO}$ is moderately reduced. Using ai-CRM with a $0.75 \mathrm{~mW}$ laser power (a relatively higher laser power is used because GO is already reduced) at $1 \mathrm{~ms}$ integration time, we observe that both $\mathrm{rGO}$ and PAA show their characteristic Raman spectra (bottom, Figure 3.12(a)), with the $\mathrm{CH}$ stretching band of PAA located at around $2930 \mathrm{~cm}^{-1}$. We subsequently scanned a vertical stack of 20 images across a depth of $10 \mu \mathrm{m}$. Each image in the stack covers $50 \times 50 \mu \mathrm{m}^{2}$ with a step size of $0.5 \mu \mathrm{m}$ and $1 \mathrm{~ms}$ integration time (Figure 3.12(a)) The imaging thus took $100 \times 100 \times 0.001 \times 20=200$ seconds of pure measurement time, and a total of $\approx 720$ s when the camera readout time and stage translation time were taken into account. Afterwards, the three dimensional distribution of rGO is plotted (Figure 3.12(b)), showing how rGO is blended into the composite. Slices extracted from any arbitrary positions within the volumetric view can be visualized, as shown by the two cross-section images in the bottom insets of Figure 3.12 (b) whose positions are labelled by the dashed green and red lines.

\subsubsection{Fast mapping of $2 \mathrm{D}$ materials on arbitrary substrates}

Compared to other characterization methods such as fluorescence quenching microscopy and bright-field optical microscopy, CRM has the advantage of universal applicability on arbitrary substrates. As an example, we used ai-CRM to image a CVD grown graphene layer on a glass substrate at $20 \mathrm{~ms}$ integration time and $1 \mathrm{~mW}$ laser power, across a $25 \times 25 \mu \mathrm{m}^{2}$ region with a step size of $0.25 \mu \mathrm{m}$ (Figure 3.13 (a),(b) and (c)). GO can also be imaged on a glass or calcium fluoride substrate with a low laser power $(10 \mu \mathrm{W})$ and short integration time $(20 \mathrm{~ms})$, as shown in Figure 

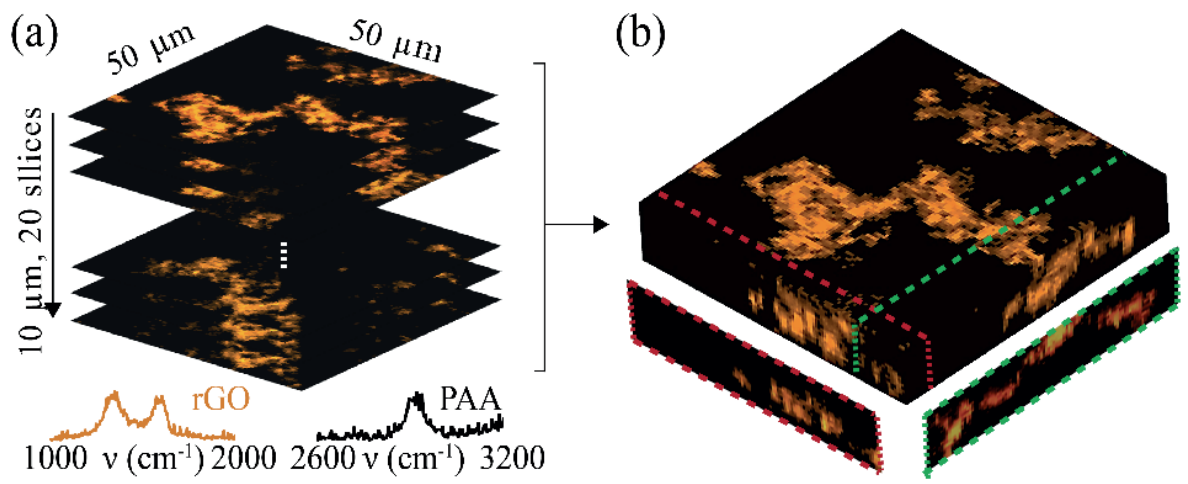

Figure 3.12: Volumetric ${ }_{3} D$ imaging of a rGO-PAA composite using ai-CRM (a) Denoised $x-y$ Raman maps of $r G O$ and PAA, taken with a step size of $0.5 \mu \mathrm{m}$. The laser power is $0.75 \mathrm{~mW}$ and integration time is $1 \mathrm{~ms}$. The spectra at the bottom correspond to $r G O$ (orange) and PAA (black), respectively. (b) ${ }_{3} D$ Raman reconstruction of the same nanocomposite, depicting the distribution of $r G O$ in the polymer matrix. The orthogonal sections along the dashed lines show the rGO flakes embedded in the matrix. Total data acquisition time: 12 minutes. 
3.13(d) and (e) respectively. The wrinkles and folds on the GO sheets are clearly visible.

\subsection{Conclusion}

In this work, we demonstrate that algorithm-improved confocal Raman microscopy (ai-CRM) significantly improves the SNR of the Raman spectra of various $2 \mathrm{D}$ nanomaterials such as graphene, GO, $\mathrm{WS}_{2}, \mathrm{MoS}_{2}$ and $\mathrm{BN}$. Thereby, it increases scanning rates by more than 50 times with respect to conventional state-of-the-art CRM (Figures $3.2(\mathrm{~h})$ and $3.6(\mathrm{~d}))$. Introducing this improvement, sensitive samples such as GO can be mapped faster at an extremely low laser power of a few $\mu \mathrm{W}$. This minimizes laser-induced sample damage and enables reliable and quantitative characterization of the physico-chemical properties of graphenic nano-sheets, such as layer number and defect density. Compared to other characterization tools, CRM has the advantage to provide label-free, substrate-independent and three-dimensional spatial information. Since the denoising performance increases with the size of the dataset, a higher scanning rate is expected when the Raman mapping area is further increased. This is an extremely useful feature as large scale industrial production of $2 \mathrm{D}$ materials and their devices require scalable characterization methods. While other techniques such as surface-enhanced Raman spectroscopy ${ }^{[60]}$ and stimulated Raman scattering ${ }^{[61]}$ may give rise to even higher SNR improvement, these techniques are either technically much more involved or require specific substrates and samples and/or do not allow for volumetric imaging. Since ai-CRM is based on a purely mathematical framework, it can also be applied to improve the above techniques instead of replacing them, as well as other hyperspectral imaging methods such as infrared microscopy and atomic force microscopy. We expect ai-CRM to strengthen the use of hyperspectral imaging as a fast, reliable, quantitative, and spatially resolved characterization tool in the fabrication and broad applications of $2 \mathrm{D}$ materials. 

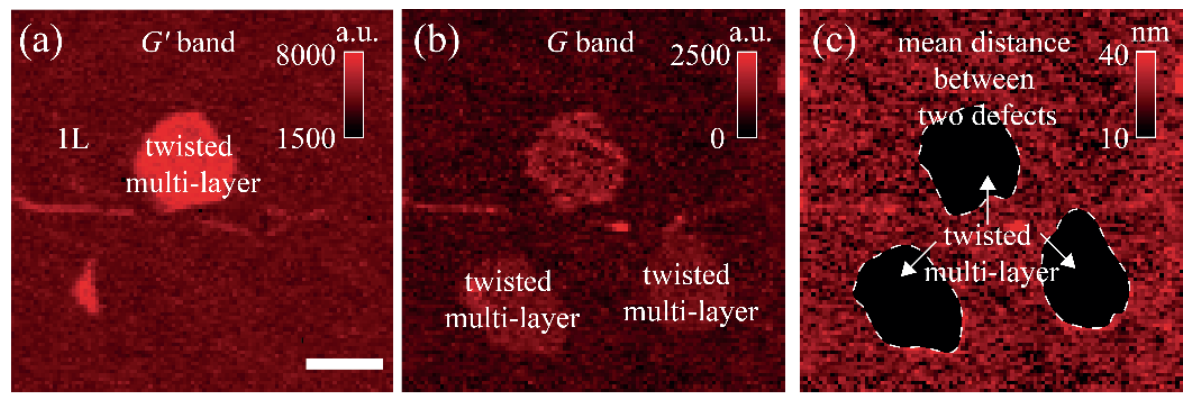

graphene on glass, $1 \mathrm{~mW}, 20 \mathrm{~ms}$

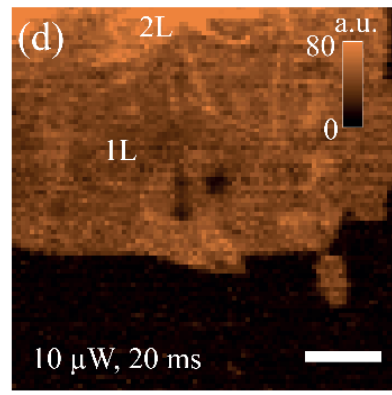

GO on glass

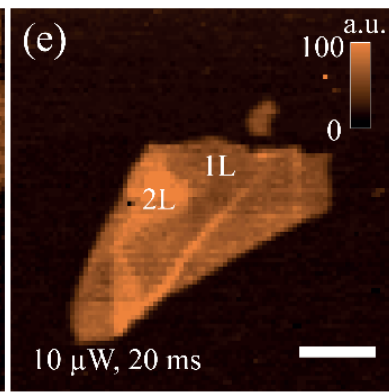

$\mathrm{GO}$ on calcium fluoride

Figure 3.13: Fast mapping of graphene and GO on arbitrary substrates with ai-CRM (a) Raman mapping of CVD grown graphene on glass integrated for the $G^{\prime}$ peak, showing the presence of only one multilayer island. (b) Raman mapping of the same region integrated for the $G$ peak, showing the presence of all the multilayer islands atop the underlying monolayer. The different maps of $G$ and $G^{\prime}$ band could be attributed to the presence of a twisted multilayer. (c) Defect density map of the same region depicting the mean defect distance, $L_{D}=30 \mathrm{~nm}$. Twisted multilayer regions are not plotted due to the possible complex layer coupling effect. Raman mapping of GO on (d) glass and (e) calcium fluoride. The laser power and integration times are mentioned in the figure and scale bars correspond to $5 \mu \mathrm{m}$. 


\section{Bibliography}

[1] A. C. Ferrari and D. M. Basko, "Raman spectroscopy as a versatile tool for studying the properties of graphene," Nature Nanotechnology, vol. 8, p. 235, 2013.

[2] X. Zhang, X.-F. Qiao, W. Shi, J.-B. Wu, D.-S. Jiang, and P.-H. Tan, "Phonon and Raman scattering of two-dimensional transition metal dichalcogenides from monolayer, multilayer to bulk material," Chemical Society Reviews, vol. 44, no. 9, pp. 2757-2785, 2015 .

[3] K. S. Novoselov, A. K. Geim, S. V. Morozov, D. Jiang, Y. Zhang, S. V. Dubonos, I. V. Grigorieva, and A. A. Firsov, "Electric field effect in atomically thin carbon films," science, vol. 306, no. 5696, pp. 666-669, 2004.

[4] D. Pacile, J. Meyer, . Girit, and A. Zettl, “The two-dimensional phase of boron nitride: Few-atomic-layer sheets and suspended membranes," Applied Physics Letters, vol. 92, no. 13, p. $133107,2008$.

[5] I. Jung, M. Pelton, R. Piner, D. A. Dikin, S. Stankovich, S. Watcharotone, M. Hausner, and R. S. Ruoff, "Simple approach for high-contrast optical imaging and characterization of graphene-based sheets," Nano Letters, vol. 7, no. 12 , pp. 3569-3575, 2007.

[6] J. Gao, W. Guo, H. Geng, X. Hou, Z. Shuai, and L. Jiang, "Layer-by-layer removal of insulating few-layer mica flakes for asymmetric ultra-thin nanopore fabrication," Nano research, vol. 5, no. 2, pp. 99-108, 2012.

[7] S. Stankovich, D. A. Dikin, R. D. Piner, K. A. Kohlhaas, A. Kleinhammes, Y. Jia, Y. Wu, S. T. Nguyen, and R. S. Ruoff, "Synthesis of graphene-based nanosheets via chemical reduction of exfoliated graphite oxide," carbon, vol. 45, no. 7 , pp. $1558-1565,2007$. 
[8] J. Kim, L. J. Cote, F. Kim, and J. Huang, "Visualizing graphene based sheets by fluorescence quenching microscopy," Journal of the American Chemical Society, vol. 132 , no. 1, pp. 260-267, 2009.

[9] J. Kim, F. Kim, and J. Huang, "Seeing graphene-based sheets," Materials today, vol. 13 , no. 3 , pp. $28-38,2010$.

[10] A. C. Ferrari, J. Meyer, V. Scardaci, C. Casiraghi, M. Lazzeri, F. Mauri, S. Piscanec, D. Jiang, K. Novoselov, and S. Roth, "Raman spectrum of graphene and graphene layers," Physical review letters, vol. 97, no. 18, p. $187401,2006$.

[1 1] A. Eckmann, A. Felten, A. Mishchenko, L. Britnell, R. Krupke, K. S. Novoselov, and C. Casiraghi, "Probing the nature of defects in graphene by Raman spectroscopy," Nano Letters, vol. 12, no. 8, pp. 3925-3930, 2012.

[12] D. Yoon, H. Moon, H. Cheong, J. S. Choi, J. A. Choi, and B. H. Park, "Variations in the Raman spectrum as a function of the number of graphene layers," J. Korean Phys. Soc, vol. 55, no. 3, pp. 1299-1303, 2009.

[13] B. Krauss, P. Nemes-Incze, V. Skakalova, L. P. Biro, K. v. Klitzing, and J. H. Smet, "Raman scattering at pure graphene zigzag edges," Nano Letters, vol. 10, no. 11, pp. 4544-4548, 2010.

[14] A. Favron, E. Gaufrès, F. Fossard, A.-L. Phaneuf-L'Heureux, N. Y. Tang, P. L. Lévesque, A. Loiseau, R. Leonelli, S. Francoeur, and R. Martel, "Photooxidation and quantum confinement effects in exfoliated black phosphorus," Nature materials, vol. 14, no. 8, p. 826, 2015 .

[15] A. C. Neto and F. Guinea, "Electron-phonon coupling and Raman spectroscopy in graphene," Physical Review B, vol. 75, no. 4, p. 045404, 2007.

[16] D. Voiry, J. Yang, J. Kupferberg, R. Fullon, C. Lee, H. Y. Jeong, H. S. Shin, and 
M. Chhowalla, "High-quality graphene via microwave reduction of solutionexfoliated graphene oxide," Science, vol. 353, no. 6306, pp. 1413-1416, 2016.

[17] L. G. Cançado, A. Jorio, E. H. M. Ferreira, F. Stavale, C. A. Achete, R. B. Capaz, M. V. O. Moutinho, A. Lombardo, T. S. Kulmala, and A. C. Ferrari, "Quantifying defects in graphene via Raman spectroscopy at different excitation energies," Nano Letters, vol. 11, no. 8, pp. 3190-3196, 2011.

[18] F. Yin, S. Wu, Y. Wang, L. Wu, P. Yuan, and X. Wang, "Self-assembly of mildly reduced graphene oxide monolayer for enhanced Raman scattering," Journal of Solid State Chemistry, vol. 237, pp. 57-63, 2016.

[19] R. V. Gorbachev, I. Riaz, R. R. Nair, R. Jalil, L. Britnell, B. D. Belle, E. W. Hill, K. S. Novoselov, K. Watanabe, and T. Taniguchi, "Hunting for monolayer boron nitride: optical and Raman signatures,” Small, vol. 7, no. 4, pp. 465-468, 2011.

[20] L. Song, L. Ci, H. Lu, P. B. Sorokin, C. Jin, J. Ni, A. G. Kvashnin, D. G. Kvashnin, J. Lou, and B. I. Yakobson, "Large scale growth and characterization of atomic hexagonal boron nitride layers," Nano letters, vol. 10, no. 8, pp. 32093215,2010 .

[21] C. Lee, H. Yan, L. E. Brus, T. F. Heinz, J. Hone, and S. Ryu, "Anomalous lattice vibrations of single-and few-layer MoS2," ACS nano, vol. 4, no. 5, pp. 2695$2700,2010$.

[22] Q.H. Wang, K. Kalantar-Zadeh, A. Kis, J. N. Coleman, and M. S. Strano, "Electronics and optoelectronics of two-dimensional transition metal dichalcogenides," Nature nanotechnology, vol. 7, no. 11, p. 699, 2012.

[23] W. Lu, H. Nan, J. Hong, Y. Chen, C. Zhu, Z. Liang, X. Ma, Z. Ni, C. Jin, and Z. Zhang, "Plasma-assisted fabrication of monolayer phosphorene and its Raman characterization," Nano Research, vol. 7, no. 6, pp. 853-859, 2014. 
[24] L. Jiao, L. Zhang, X. Wang, G. Diankov, and H. Dai, "Narrow graphene nanoribbons from carbon nanotubes," Nature, vol. 458, p. 877, 2009.

[25] L. Zhang, J. Yu, M. Yang, Q. Xie, H. Peng, and Z. Liu, "Janus graphene from asymmetric two-dimensional chemistry," Nature communications, vol. 4, p. $1443,2013$.

[26] X. Wang and H. Dai, "Etching and narrowing of graphene from the edges," Nature Chemistry, vol. 2, p. 661, 2010.

[27] R. Yang, Z. Shi, L. Zhang, D. Shi, and G. Zhang, "Observation of Raman Gpeak split for graphene nanoribbons with hydrogen-terminated zigzag edges," Nano Letters, vol. 11, no. 10, pp. 4083-4088, 2011.

[28] Z. Sun, C. L. Pint, D. C. Marcano, C. Zhang, J. Yao, G. Ruan, Z. Yan, Y. Zhu, R. H. Hauge, and J. M. Tour, "Towards hybrid superlattices in graphene," Nature Communications, vol. 2, p. 559, 2011.

[29] J. E. Lee, G. Ahn, J. Shim, Y. S. Lee, and S. Ryu, “Optical separation of mechanical strain from charge doping in graphene," Nature Communications, vol. 3, p. $1024,2012$.

[3o] H. Nakajima, T. Morimoto, Y. Okigawa, T. Yamada, Y. Ikuta, K. Kawahara, H. Ago, and T. Okazaki, "Imaging of local structures affecting electrical transport properties of large graphene sheets by lock-in thermography," Science Advances, vol. 5, no. 2, p. eaau3407, 2019.

[31] Y. Stubrov, A. Nikolenko, V. Strelchuk, S. Nedilko, and V. Chornii, "Structural modification of single-layer graphene under laser irradiation featured by microRaman spectroscopy," Nanoscale research letters, vol. 12, no. 1, p. 297, 2017.

[32] S. Wagner, T. Dieing, A. Centeno, A. Zurutuza, A. D. Smith, M. Östling, S. Kataria, and M. C. Lemme, "Non-invasive scanning Raman spectroscopy 
and tomography for graphene membrane characterization," Nano Letters, vol. 17, no. 3, pp. 1504-1511, 2017.

[33] R. W. Havener, S.-Y. Ju, L. Brown, Z. Wang, M. Wojcik, C. S. Ruiz-Vargas, and J. Park, "High-throughput graphene imaging on arbitrary substrates with widefield Raman spectroscopy," ACS Nano, vol. 6, no. 1, pp. 373-380, 2012.

[34] J. S. Mehta, A. C. Faucett, A. Sharma, and J. M. Mativetsky, "How reliable are Raman spectroscopy measurements of graphene oxide?," The Journal of Physical Chemistry C, vol. 121, no. 30, pp. 16584-16591, 2017.

[35] M. Unser and A. Aldroubi, "A review of wavelets in biomedical applications," Proceedings of the IEEE, vol. 84, no. 4, pp. 626-638, 1996.

[36] D. Shen, G. Wu, and H.-I. Suk, "Deep learning in medical image analysis," Annual review of biomedical engineering, vol. 19, pp. 221-248, 2017.

[37] C. Kallepitis, M. S. Bergholt, M. M. Mazo, V. Leonardo, S. C. Skaalure, S. A. Maynard, and M. M. Stevens, "Quantitative volumetric Raman imaging of three dimensional cell cultures," Nature communications, vol. 8, p. 14843, 2017.

[38] H. Abdi and L. J. Williams, "Principal component analysis," Wiley interdisciplinary reviews: computational statistics, vol. 2, no. 4, pp. 433-459, 2010.

[39] N. Uzunbajakava, A. Lenferink, Y. Kraan, E. Volokhina, G. Vrensen, J. Greve, and C. Otto, "Non-resonant confocal Raman imaging of DNA and protein distribution in apoptotic cells," Biophysical journal, vol. 84, no. 6, pp. 3968-3981, 2003.

[40] H.-J. van Manen, Y. M. Kraan, D. Roos, and C. Otto, "Intracellular chemical imaging of heme-containing enzymes involved in innate immunity using resonance Raman microscopy," The Journal of Physical Chemistry B, vol. 108, no. 48, pp. $18762-18771,2004$. 
[41] S. Valle, W. Li, and S. J. Qin, "Selection of the number of principal components: the variance of the reconstruction error criterion with a comparison to other methods," Industrial and Engineering Chemistry Research, vol. 38, no. 11, pp. 4389-4401, 1999.

[42] M. Rogala, P. Dabrowski, P. J. Kowalczyk, I. Wlasny, W. Kozlowski, A. Busiakiewicz, I. Karaduman, L. Lipinska, J. M. Baranowski, and Z. Klusek, "The observer effect in graphene oxide - how the standard measurements affect the chemical and electronic structure," Carbon, vol. 103, pp. 235-241, 2016.

[43] S. Eigler, C. Dotzer, and A. Hirsch, "Visualization of defect densities in reduced graphene oxide," Carbon, vol. 50, no. 10, pp. 3666-3673, 2012.

[44] D. Yang, A. Velamakanni, G. Bozoklu, S. Park, M. Stoller, R. D. Piner, S. Stankovich, I. Jung, D. A. Field, C. A. Ventrice, and R. S. Ruoff, "Chemical analysis of graphene oxide films after heat and chemical treatments by x-ray photoelectron and micro-Raman spectroscopy," Carbon, vol. 47, no. 1, pp. 145-152, 2009.

[45] X. Díez-Betriu, S. Álvarez García, C. Botas, P. Álvarez, J. Sánchez-Marcos, C. Prieto, R. Menéndez, and A. de Andrés, "Raman spectroscopy for the study of reduction mechanisms and optimization of conductivity in graphene oxide thin films," Journal of Materials Chemistry C, vol. 1, no. 41, pp. 6905-6912, 2013.

[46] D. Kostiuk, M. Bodik, P. Siffalovic, M. Jergel, Y. Halahovets, M. Hodas, M. Pelletta, M. Pelach, M. Hulman, Z. Spitalsky, M. Omastova, and E. Majkova, "Reliable determination of the few-layer graphene oxide thickness using Raman spectroscopy," Journal of Raman Spectroscopy, vol. 47, no. 4, pp. 391-394, 2016.

[47] P. Vecera, S. Eigler, M. Koleśnik-Gray, V. Krstić, A. Vierck, J. Maultzsch, R. A. Schäfer, F. Hauke, and A. Hirsch, "Degree of functionalisation dependence 
of individual Raman intensities in covalent graphene derivatives," Scientific Reports, vol. 7, p. 45165, 2017.

[48] K. W. Silverstein, C. E. Halbig, J. S. Mehta, A. Sharma, S. Eigler, and J. M. Mativetsky, "Voltage-reduced low-defect graphene oxide: a high conductivity, nearzero temperature coefficient of resistance material," Nanoscale, 2019.

[49] H. Yang, H. Hu, Y. Wang, and T. Yu, "Rapid and non-destructive identification of graphene oxide thickness using white light contrast spectroscopy," Carbon, vol. 52, pp. 528-534, 2013.

[5o] J. Huang, C. Zong, H. Shen, M. Liu, B. Chen, B. Ren, and Z. Zhang, "Mechanism of cellular uptake of graphene oxide studied by surface-enhanced Raman spectroscopy," Small, vol. 8, no. 16, pp. 2577-2584, 2012.

[51] S. Eigler, F. Hof, M. Enzelberger-Heim, S. Grimm, P. Müller, and A. Hirsch, "Statistical Raman microscopy and atomic force microscopy on heterogeneous graphene obtained after reduction of graphene oxide," The Journal of Physical Chemistry C, vol. 118, no. 14, pp. 7698-7704, 2014.

[52] X. Li, W. Cai, J. An, S. Kim, J. Nah, D. Yang, R. Piner, A. Velamakanni, I. Jung, E. Tutuc, S. K. Banerjee, L. Colombo, and R. S. Ruoff, "Large-area synthesis of high-quality and uniform graphene films on copper foils," Science, vol. 324, no. 5932, pp. 1312-1314, 2009.

[53] M. M. MacInnes, S. Hlynchuk, S. Acharya, N. Lehnert, and S. Maldonado, "Reduction of graphene oxide thin films by cobaltocene and decamethylcobaltocene," ACS Applied Materials and Interfaces, vol. 10, no. 2, pp. 2004-2015, 2018.

[54] H. Wang, J. T. Robinson, X. Li, and H. Dai, "Solvothermal reduction of chemically exfoliated graphene sheets," Journal of the American Chemical Society, vol. 131, no. 29, pp. 9910-9911, 2009. 
[55] C. Tyagi, S. A. Khan, I. Sulania, R. Meena, D. K. Avasthi, and A. Tripathi, "Evidence of ion-beam-induced annealing in graphene oxide films using in situ x-ray diffraction and spectroscopy techniques," The Journal of Physical Chemistry C, vol. 122, no. 17, pp. 9632-9640, 2018.

[56] S. Barg, F. M. Perez, N. Ni, P. do Vale Pereira, R. C. Maher, E. Garcia-Tuñon, S. Eslava, S. Agnoli, C. Mattevi, and E. Saiz, "Mesoscale assembly of chemically modified graphene into complex cellular networks," Nature Communications, vol. 5 , p. 4328,2014 .

[57] H. Liu, S. Ryu, Z. Chen, M. L. Steigerwald, C. Nuckolls, and L. E. Brus, "Photochemical reactivity of graphene," Journal of the American Chemical Society, vol. 131, no. 47, pp. 17099-17101, 2009.

[58] Y. Ito, Y. Tanabe, H. J. Qiu, K. Sugawara, S. Heguri, N. H. Tu, K. K. Huynh, T. Fujita, T. Takahashi, K. Tanigaki, and M. Chen, "High-quality threedimensional nanoporous graphene," Angewandte Chemie International Edition, vol. 53, no. 19, pp. 4822-4826, 2014 .

[59] L. Jiao, X. Wang, G. Diankov, H. Wang, and H. Dai, "Facile synthesis of highquality graphene nanoribbons," Nature Nanotechnology, vol. 5, p. 32 1, 2010.

[6o] A. Campion and P. Kambhampati, "Surface-enhanced Raman scattering," Chemical society reviews, vol. 27, no. 4, pp. 241-250, 1998.

[61] R. C. Prince, R. R. Frontiera, and E. O. Potma, "Stimulated Raman scattering: from bulk to nano," Chemical reviews, vol. 117, no. 7, pp. 5070-5094, 2016. 
"If you wear a wig, everybody notices. But if you then dye the wig, people notice the dye."

Andy Warhol

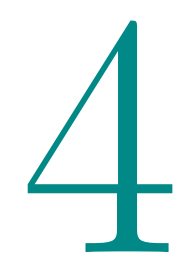

\section{Ultra-sensitive detection and in-situ imaging of analytes on graphene oxide analogues using enhanced Raman Spectroscopy ${ }^{\dagger}$}

WE DEMONSTRATE HOW algorithm-improved confocal Raman microscopy (aiCRM), in combination with chemical enhancement by two-dimensional substrates, can be used as an ultra-sensitive detection method for rhodamine (R6G) molecules adsorbed from aqueous solutions. After developing a protocol for laser-induced re-

${ }^{\dagger}$ Published as: Nair, Sachin et al., Analytical chemistry 93.38 (2021): 12966-12972. 
duction of graphene oxide (rGO), followed by non-invasive Raman imaging, a limit of detection (LOD) of $5 \times 10^{-10} \mathrm{M}$ R6G was achieved using ai-CRM. An equivalent sub-nanomolar LOD was also achieved on another graphene oxide analogue UV/ozone-oxidized graphene (oG). These record-breaking detection capabilities also enabled us to study the adsorption kinetics and image the spatial distribution of the adsorbed R6G. These findings indicate a strong potential for algorithm-improved Graphene-Enhanced Raman Spectroscopy (“ai-GERS”) as a facile method for detecting, imaging and quantifying trace amounts of adsorbing molecules on a variety of $2 \mathrm{D}$ substrates.

\subsection{Introduction}

Graphene-enhanced Raman spectroscopy (GERS) ${ }^{[1]}$, a subset of surface-enhanced Raman spectroscopy (SERS $)^{[2,3]}$, refers to the use of graphenic two-dimensional (2D) materials as substrates for enhancing the weak Raman scattering cross-section of adsorbed analyte molecules ${ }^{[4]}$. Utilizing pristine graphene to suppress the fluorescence of adsorbed rhodamine- $6 \mathrm{~g}$ molecules in resonance Raman spectroscopy ${ }^{[5]}, \mathrm{Xi}$ Ling et al. were the first to demonstrate the potential of graphene as a substrate for Raman enhancement ${ }^{[1]}$. Compared to plasmonic nanoparticle-based substrates typically used in SERS, graphenic materials exhibit excellent bio-compatibility, chemical inertness, reproducibility, ease of fabrication and low costs ${ }^{[6]}$. The atomically flat surface of graphene, endowed with a highly delocalized $\pi$-electronic system, provides a very uniform adsorption template for a variety of conjugated probe molecules ${ }^{[4]}$. Contrary to substrate materials that support surface plasmon resonance in the visible range of the electromagnetic spectrum, the Raman enhancement in GERS arises from weak surface-analyte charge transfer interactions, also known as the chemical-enhanced mechanism ${ }^{[7,8]}$. The absence of intense electromagnetic "hot-spots" on graphene and its inert nature also mitigate the photo-degradation of the adsorbed molecule, resulting in higher stability of the Raman signal ${ }^{[9]}$. The 
aforementioned properties of graphene thus eliminate the problems of irreproducibility and spectral instability that are commonly encountered in SERS ${ }^{[10]}$. Additionally the chemical enhancement in GERS can be fine-tuned by controlling the interaction distance between the analyte and graphene ${ }^{[7]}$, the applied-voltage dependent fermi-level shift of graphene ${ }^{[11,12]}$, the type of chemical doping ${ }^{[13,12,14,15]}$, the size of the graphitic domains ${ }^{[16]}$ and the molecular structure and energy levels of the analyte $^{[17]}$.

While initial reports focused on the optimization of graphene as a GERS substrate, similar chemical enhancements have also been reported for other graphenic materials $^{[18,19]}$. Graphene Oxide $(\mathrm{GO})$, the oxidized version of graphene, has certain advantages as a GERS substrate. It can be stably dispersed in water, readily coated on any surface by simple drop-casting and has a rich surface chemistry which can be modulated by varying the degree of oxidation of graphene ${ }^{[20]}$ or the degree of reduction of $\mathrm{GO}^{[21]}$. The controlled reduction of $\mathrm{GO}$ to reduced graphene oxide (rGO) not only improves its electrical conductivity due to partial recovery of graphene-like domains ${ }^{[22,21]}$, but also enables it to act as an efficient GERS substrate ${ }^{[23,24]}$. rGO can be readily produced from GO by chemical treatment, thermal or laser annealing ${ }^{[21]}$. Even though chemical and thermal treatments are most often used to produce $\mathrm{rGO}$, the former can introduce reagent-induced impurities into the rGO lattice $^{[25]}$, whereas the latter is highly energy intensive ${ }^{[26]}$. Compared to these, laserannealed fabrication of $\mathrm{rGO}^{[27]}$ is facile, energy efficient and chemical-free, and provides excellent control over the degree of photo-reduction by simple tuning of the incident laser dosage ${ }^{[28,29]}$.

Determining the limit of detection (LOD) of macrocyclic fluorescent molecules like rhodamine-6g (R6G) is one of the most common ways to judge the performance of GERS substrates ${ }^{[30,31]}$. For instance, a recent study reported the detection and Raman imaging of R6G layers dried onto graphene quantum dots, starting from aqueous droplets with R6G concentrations as low as $1 \times 10^{-9} \mathrm{M}^{[16]}$. However, 
most often the probing of trace amounts of analytes in GERS-based chemical and bio-sensing applications is severely limited by a low signal-to-noise ratio (SNR). As a mitigation, a relatively high laser dosage (exposure time per pixel $\sim 10 \mathrm{~s}$, laser power $\sim 1 \mathrm{~mW})$ is often used when probing low analyte concentrations $\left(<10^{-6} \mathrm{M}\right)$. However, such a dosage can (a) affect the photo-stability of the adsorbed fluorescent molecules ${ }^{[9]},(\mathrm{b})$ compromise the integrity of photo-sensitive substrates like $\mathrm{GO}^{[32,33]}$, and (c) still involve long exposure times per pixel, making it unattractive to collect large-area Raman maps, as often needed for obtaining statistically sound and reproducible results. Recently it was indicated that such problems could be avoided by using a very low laser dosage combined with so-called algorithm-improved confocal Raman microscopy (ai-CRM) ${ }^{[33]}$. ai-CRM can improve the SNR of collected Raman hyperspectral datasets using a robust and efficient denoising algorithm based on principal component analysis (PCA). Thanks to the improved SNR, the method offers the potential to perform fast and non-invasive Raman imaging and reliable quantification, even for photo-sensitive systems.

In this work, we show that significant improvements in the detection of R6G molecules on monolayer graphene oxide analogues can be achieved by implementing algorithm-improved GERS. After establishing the range of laser powers where photodamage is avoided, we use ai-GERS to assess the efficiency of photo-reduced GO as a Raman enhancement substrate and demonstrate that the limits of detection of R6G molecules on rGO and oxidized graphene substrates are indeed significantly improved. We then use this highly sensitive technique to monitor the in-situ adsorption kinetics of R6G on rGO. Lastly, we demonstrate an application of rGO as a sensor for detecting trace adulteration in commercial fruit juice. 


\subsection{Experimental section}

\section{Materials}

A $50 \mu \mathrm{L}$ GO suspension ( $2 \mathrm{mg} \mathrm{mL}^{-1}, 22 \mu \mathrm{m}$ mean diameter flakes, Sigma Aldrich) was diluted with $30 \mathrm{~mL}$ of deionized water (Millipore, resistivity $18.2 \mathrm{M} \Omega \mathrm{cm}$ ). 100 $\mu \mathrm{L}$ of this diluted suspension was deposited on $1 \mathrm{~cm} \times 1 \mathrm{~cm}$ plasma-cleaned 300$\mathrm{nm}-\mathrm{SiO}_{2} / \mathrm{Si}$ wafers (Okmetic) by drop-casting. After drying in a vacuum oven at $35^{\circ} \mathrm{C}$, the substrate showed partial coverage of single-layer GO flakes, which were optically selected for performing laser writing. Monolayer CVD-grown graphene supported on $1 \mathrm{~cm} \times 1 \mathrm{~cm}, 300-\mathrm{nm}-\mathrm{SiO}_{2} / \mathrm{Si}$ substrates (Graphene supermarket, USA) were cleaned with deionized water and gently dried under a nitrogen flow. Next, the graphene samples were subjected to oxidation in a UV-Ozone cleaner (Bioforce Nanosciences, ProCleaner ${ }^{\mathrm{TM}}$ Plus) for 5 minutes, resulting in a large chemical enhancement factor for GERS measurements ${ }^{[13]}$. The oxidized-graphene (oG) substrates were then used for performing ai-GERS. Aqueous solutions of rhodamine- $6 \mathrm{~g}$ (Merck, 99\% dye content) of different concentrations were prepared by diluting a stock solution of $1 \times 10^{-3} \mathrm{M}$. Commercial fruit-extract based juice was purchased and centrifuged to remove the heavy fruit particulates. The supernatant was then adulterated with R6G such that the final concentration is $50 \mathrm{nM}$ (solvent-juice) for in-situ ai-GERS measurements on rGO.

\section{Raman measurements and imaging}

Raman measurements were carried out using a WiTec alpha 300R Raman microscope equipped with a $532 \mathrm{~nm}$ laser. A $600 \mathrm{~g} / \mathrm{mm}$ (wavenumber range $=0-3710$ $\mathrm{cm}^{-1}$ ) or $1800 \mathrm{~g} / \mathrm{mm}$ (wavenumber range $=650-1705 \mathrm{~cm}^{-1}$ ) grating was used as the dispersing element and a CCD camera $(1600 \times 200$ pixels, $16 \mu \mathrm{m}$ pixel size, Andor Newton) was used for detection of the Raman scattered photons. For performing measurements on the substrate through the ambient deionized water/R6G solution, a $63 x$ water dipping objective (Zeiss W “N-Achroplan”, Numerical Aperture (NA) 
0.9 , working distance $=2.4 \mathrm{~mm}$ ) was chosen. The laser power was measured before the objective (at the turret) using an optical power meter (ThorLabs) and the values are mentioned accordingly in the chapter.

For a $532 \mathrm{~nm}$ laser excitation, the characteristic Raman bands of R6G appear at 610 $\mathrm{cm}^{-1}$ (in-plane bending of the xanthene ring), $772 \mathrm{~cm}^{-1}$ (out-of-plane bending of $\mathrm{C}-\mathrm{H}$ ) and $1649 \mathrm{~cm}^{-1}$ (aromatic C-C stretch). Within the wavenumber resolution of the diffraction grating $\left(2.3 \mathrm{~cm}^{-1}\right)$, the values are in good agreement with previous reports ${ }^{[1,5]}$. The Si-Si crystalline vibrations from the underlying silicon substrate appear at $520 \mathrm{~cm}^{-1}$. For graphene oxide (GO), characteristic broad D and $\mathrm{G}$ bands appear at $1352 \mathrm{~cm}^{-1}$ and $1597 \mathrm{~cm}^{-1}$ respectively, whereas for graphene the $\mathrm{G}$ peak is located at $1590 \mathrm{~cm}^{-1}$.

Laser writing on GO was performed at varying laser power $\left(\mathrm{I}_{\mathrm{W}}=0\right.$ to $\left.2 \mathrm{~mW}\right)$ and using an exposure time per pixel, $\mathrm{t}_{\mathrm{W}}=0.1 \mathrm{~s}$. Individual square domains of $\mathrm{rGO}$ were written over an area of $15 \times 15 \mu \mathrm{m}^{2}$ and at a resolution of $20 \times 20$ pixels. In this manner, 6 patterns with varying degrees of photo-reduction were created on the same GO flake. Multiple writing experiments $(n=4)$ were performed to ensure reproducibility.

For ai-CRM (ai-GERS), the collected Raman image dataset was subjected to cosmic ray removal and principal component analysis (PCA) using home-written codes on MATLAB (version R2017b). Denoising was performed by retaining the first $\mathrm{k}$ principal components that explained the maximum variance in the data, during the reconstruction. For more details on the technique, its optimization and the MATLAB code for denoising, readers are encouraged to refer to the original work by Nair et al. ${ }^{[33]}$ or chapter 2 of this thesis. Raman imaging was performed by integrating the band of interest in the denoised spectrum for every mapped pixel: from 580-670 $\mathrm{cm}^{-1}$ for $A_{610}, 730-805 \mathrm{~cm}^{-1}$ for $A_{772}$ and $1620-1675 \mathrm{~cm}^{-1}$ for $A_{1649}$ of R6G, and $480-580 \mathrm{~cm}^{-1}$ for $A_{S i}$ of silicon which is used for normalization. Fluorescence area, $A_{\text {Fluo }}$ is determined by calculating the area under the curved baseline (excluding peak 
areas) of the Raman spectrum, between $0-3692 \mathrm{~cm}^{-1}$. A dark measurement $\left(\mathrm{I}_{\mathrm{P}}=\right.$ o $\mathrm{mW}$ ) was used as the reference background spectrum. Typical Raman maps are made with a resolution of $0.67 \mu \mathrm{m} /$ pixel, which approximately corresponds to the diffraction-limited spatial resolution of the objective and laser used.

\subsection{Results and Discussion}

\subsection{1 rGO as a GERS substrate}

rGO substrates were fabricated by in-situ photo-reduction of $\mathrm{GO}$ nanosheets via laser writing ${ }^{[28][29]}$. First, an as-purchased aqueous GO suspension is drop-cast on a $300-\mathrm{nm}-\mathrm{SiO}_{2} / \mathrm{Si}$ substrate and dried. The GO coated substrate is then immersed in $20 \mathrm{~mL}$ of deionized (DI) water in a liquid cell. A $532 \mathrm{~nm}$ laser, focused through a $63 \mathrm{x}, 0.9 \mathrm{NA}$, water-dipping objective is used to write $15 \times 15 \mu \mathrm{m}^{2}$ square patterns with different writing powers ( $\mathrm{I}_{\mathrm{W}}=0.1$ to $2 \mathrm{~mW}$ ), and hence varying rates of photoreduction, on a single GO flake as shown in Figure 4.1 (a). Atomic Force Microscopy (AFM) characterization of a GO flake patterned in this way shows the presence of a single layer (thickness $\sim 1.0 \mathrm{~nm}$ ), with no difference in topography between the rGO and GO regions (Figure 4.2(a)-(d)). As $\mathrm{I}_{\mathrm{W}}$ increases, the D to G Raman band ratio (ID/IG) of the laser-illuminated area also increases, which confirms the existence of rGO domains with varying degrees of photo-reduction ${ }^{[32]}$ (Figure 4.2(e-g)). Next (Figure 4.1(b)), rhodamine-6g (R6G) is added to the DI water such that the final R6G concentration, $[\mathrm{R} 6 \mathrm{G}]$ is $5 \times 10^{-8} \mathrm{M}$. After an incubation time of 2 hours, the spatial distribution of $\mathrm{R} 6 \mathrm{G}$ on the substrate is probed using the same optics but a low laser power, $\mathrm{I}_{\mathrm{P}}=0.1 \mathrm{~mW}$ and an exposure time, $\mathrm{t}_{\mathrm{P}}=0.2 \mathrm{~s} / \mathrm{pixel}$ (Figure 4.1(c)). Using the water-immersion objective allows us to probe the analyte adsorption in-situ and thereby avoids potential artefacts of common procedures involving intermediate drying steps.

Representative Raman spectra (Figure 4.1(d)) obtained from a single pixel over the 
(a) laser writing on GO

(b) R6G adsorption, 2h

(c) probing adsorbed R6G

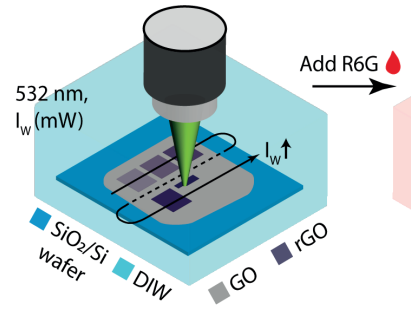

(d) GERS spectrum vs. $I_{w}$

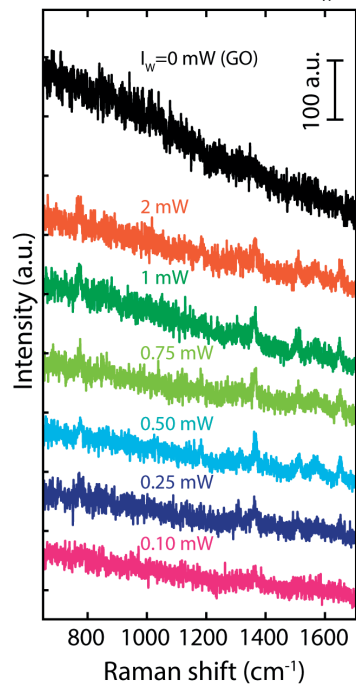

(e) ai-GERS spectrum vs. I

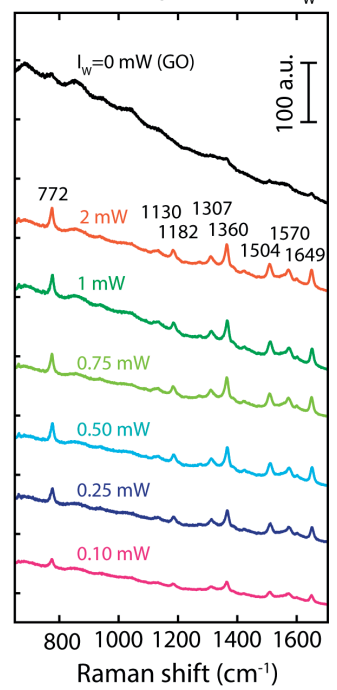

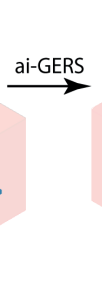

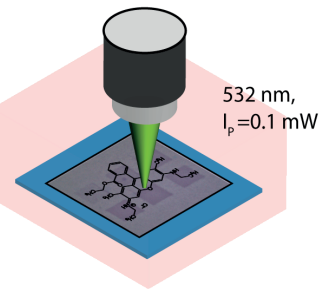

(f)

R6G Raman map, $\mathrm{A}_{1649}$
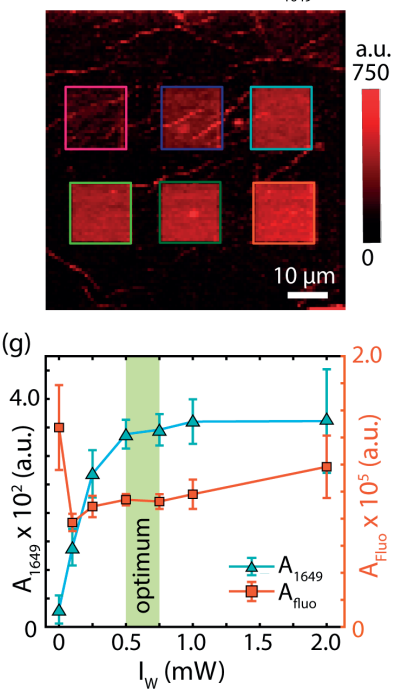

Figure 4.1: $(a, b, c)$ Schematic of the subsequent steps in our experiment. (a) in-situ laser writing on $G O$ to create $r G O$ domains with varying degree of photo-reduction through variation of the writing power $I_{W},(b) R 6 G$ adsorption on the patterned GO flake from $a$ bulk solution with $[R 6 G]=5 \times 10^{-8} M$ and (c) measurement of the adsorbed R6G distribution using GERS. (d) representative GERS spectrum (single pixel) as a function of $I_{W}$. (e) representative corresponding ai-GERS spectrum vs. $I_{W}$, showing the improved SNR. The characteristic band positions of $R 6 G$ are labelled and the spectra have been vertically offset for clarity. $(f)$ Raman map of the adsorbed R6G obtained by integrating across the band at $1649 \mathrm{~cm}^{-1}$. (g) Variation of the mean intensities $A_{1649}$ and $A_{\text {Fluo }}$ (integrated over the entire spectrum baseline) with $I_{W}$. The optimum $I_{W}$ (shaded region) is chosen based on $A_{1649}$ being maximal and the spatial variations of $A_{1649}$ and $A_{\text {Fluo }}$ (error bars) being minimal. Scale bars: $10 \mu \mathrm{m}$. 
(a) optical micrograph

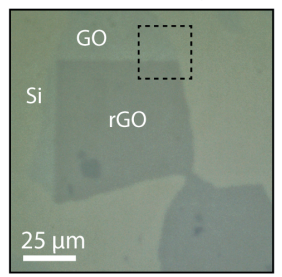

(b) topography in air

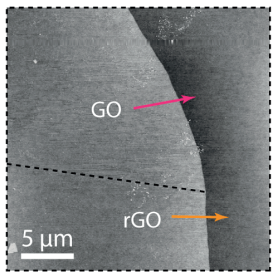

(e) optical micrograph

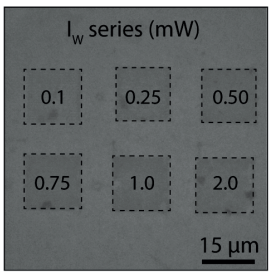

(f) $\mathrm{I}_{\mathrm{D}} / \mathrm{I}_{\mathrm{G}}$ Raman map

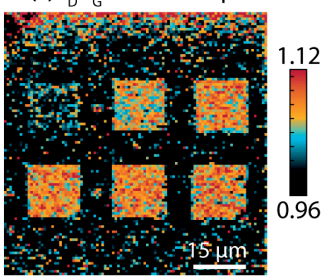

(c) topography in DIW

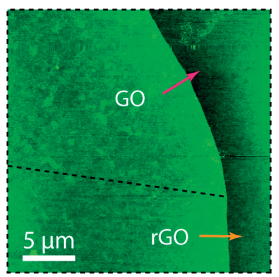

(d) height profiles

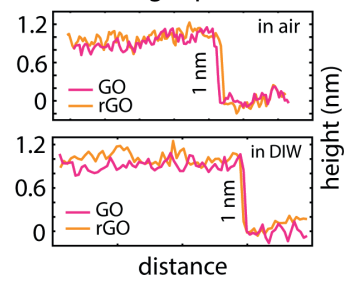

(g)

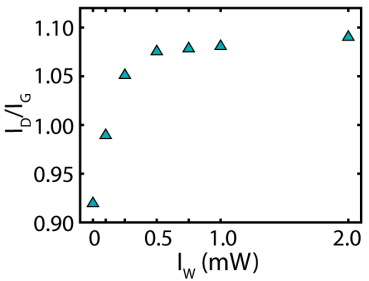

Figure 4.2: (a-d) AFM characterization of a photo-reduced GO flake ( $\left.I_{W}=0.5 \mathrm{~mW}\right)$. (a) Bright-field reflection image of a photo-reduced GO flake. The reduced region ( $G O)$ has a higher optical contrast as compared to the GO region. The dashed box indicates the window probed with AFM. (b) AFM topography image ( $512 \times 512$ pixels) in ambient air and (c) in ambient DI water. The dashed black line demarcates $r G O$ from GO. (d) Corresponding height profiles across the coloured lines shown in (b) and (c). The rGO and $G O$ regions display the same height $\left(\sim_{1} \mathrm{~nm}\right)$, both in air and DI water.

(e-g) Raman characterization of a GO flake patterned with varying $I_{W}$ (o to $2 \mathrm{~mW}$ ). (e) Bright-field reflection image of a patterned GO flake. The $I_{W}(m W)$ used to write the different $15 \times 15 \mu \mathrm{m}^{2}$ rGO domains are labelled. $(f)$ Corresponding ID/IG ratio Raman map of the same flake. The ratios are calculated by fitting the $D\left(1352 \mathrm{~cm}^{-1}\right)$ and $G(1597$ $\mathrm{cm}^{-1}$ ) Raman bands of GO with single Gaussian curves and determining their amplitude ratios for each probed pixel. ( $g$ ) The variation of the mean ID/IG ratio (averaged over their corresponding $r G O$ domain) with $I_{W}$. Note that this trend correlates excellently with the $A_{1649}$ vs. $I_{W}$ variation (Figure $4.1(\mathrm{~g})$ ) after $R 6 G$ adsorption on such a patterned GO flake. 
$15 \times 15 \mu \mathrm{m}^{2}$ reduced areas display a clear dependence on the laser writing power. Clearly, these conventionally obtained GERS spectra suffer from poor SNR, especially when dealing with minimally invasive probing conditions (probe laser dose $=4.89 \times 10^{7} \mathrm{~J} / \mathrm{m}^{2}$ ) and trace analyte concentrations. To tackle this problem, we employ an adaptation of a previously established technique ${ }^{[33]}$, which we henceforth denote as algorithm-improved graphene-enhanced Raman spectroscopy (aiGERS). The ai-mode of the technique relies on principal component analysis [34] to reduce the noise of the collected Raman data and to improve the visibility of the low-amplitude bands (compare Figure 4.1(e) vs. (d)). While the spectrum on unreduced GO is completely dominated by unspecific fluorescence (black curves in Figure 4.1(d) and (e)), the characteristic Raman peaks of R6G are clearly visible above the noise for the different probing areas reduced at variable writing power (coloured curves). Probing the reason for observing a higher Raman enhancement on $\mathrm{rGO}$ as compared to GO is outside the scope of the current chapter, but we speculate that this difference could be attributed to a larger adsorption of R6G on rGO, and/or a larger Raman scattering cross-section of the R6G-rGO complex than that of the R6G-GO complex.

The enhanced capabilities of ai-GERS are further visualized by the Raman map of the R6G distribution (Figure 4.1 (f)), obtained by integrating across the $1649 \mathrm{~cm}^{-1}$ band of R6G $\left(A_{1649}\right)$ for every probed pixel. The mean $A_{1649}$ intensity of R6G is found to increase monotonically for low $\mathrm{I}_{\mathrm{W}}$ until it saturates beyond $\sim 0.75 \mathrm{~mW}$ (Figure 4.1 $(\mathrm{g}))$. Interestingly, this trend correlates very well with the increase in the ID/IG ratio of $(\mathrm{r}) \mathrm{GO}$ with $\mathrm{I}_{\mathrm{W}}$ (Figure 4.2(g)). In contrast, the integrated fluorescence intensity $\left(\mathrm{A}_{\mathrm{Fluo}}\right)$ drops by approximately 50\% for the lowest $\mathrm{I}_{\mathrm{W}}$ and slightly increases again for high $\mathrm{I}_{\mathrm{W}}$. The combined trends indicate an optimum writing power of 0.5 to $0.75 \mathrm{~mW}$ (laser dose $=1.22$ to $1.83 \times 10^{8} \mathrm{~J} / \mathrm{m}^{2}$ ), generating maximum SNR and minimal spatial variation across the corresponding rGO domains. Technical details of the Raman measurements and vibrational band assignments can be found in the 

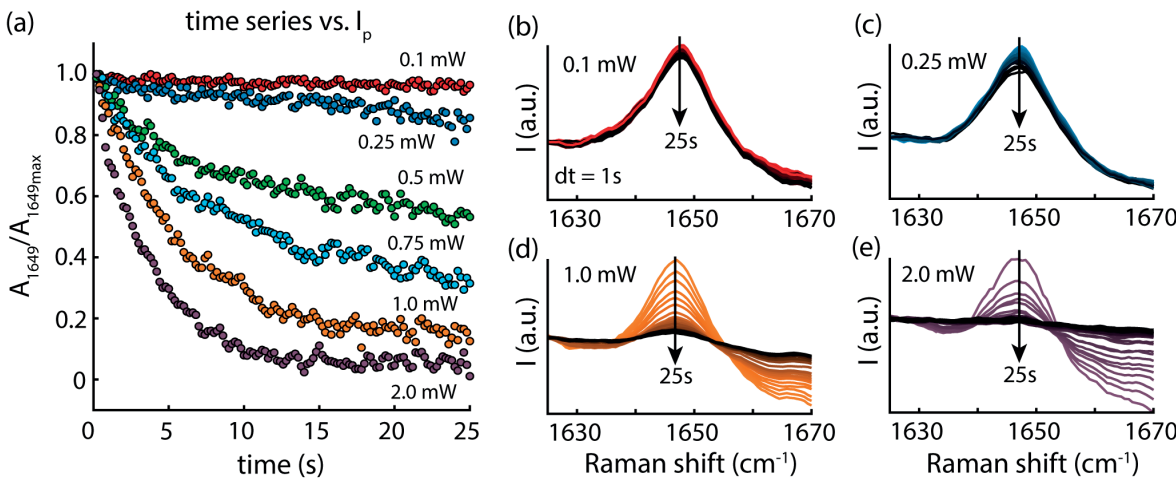

Figure 4.3: (a) Single pixel time evolution of the $A_{1649}$ intensity (normalized by its value at $t_{P}=0 \mathrm{~s}$ ) of an adsorbed R6G layer $([R 6 G]=50 \mathrm{nM})$ on $r G O$ (created with $I_{W}=0.5$ $\mathrm{mW})$ at different probing powers $\left(I_{P}\right)$. The temporal variation of the $1649 \mathrm{~cm}^{-1}$ band of R6G is shown in (b) for $I_{P}=0.1 \mathrm{~mW}$, (c) for $I_{P}=0.25 \mathrm{~mW},(d)$ for $I_{P}=1.0 \mathrm{~mW}$ and (e) for $I_{P}=2.0 \mathrm{~mW}$. For low probe laser powers $(\leq 0.25 \mathrm{~mW})$ and short exposure times (< $1 \mathrm{~s} /$ pixel), the photo-degradation-induced Raman band amplitude loss of the R6G molecules is minimal, as seen in (b) and (c). ai-GERS facilitated improvement in SNR allows for making Raman images using these minimally invasive conditions, such that the photo-stability of the probed analyte is maintained.

experimental section of this chapter.

\subsubsection{Limit of detection on rGO}

Using these optimized reduction conditions $\left(\mathrm{I}_{\mathrm{W}}=0.5 \mathrm{~mW}\right)$, we determined the limit of detection (LOD) of R6G on single rGO layers. To minimize laser induced changes in the sample while collecting the R6G spectra, we chose a nominal probing laser power, $\mathrm{I}_{\mathrm{P}}=0.25 \mathrm{~mW}$ and a short exposure time per pixel, $\mathrm{t}_{\mathrm{P}}=0.2 \mathrm{~s}$. The corresponding total laser dosage of $1.22 \times 10^{8} \mathrm{~J} / \mathrm{m}^{2}$ was verified to maintain the photostability of the adsorbed R6G molecules (Figure 4.3), while still providing sufficient SNR to probe the adsorption from very dilute solutions $\left([R 6 G] \leq 10^{-7} \mathrm{M}\right)$.

Figure 4.4 demonstrates the application of rGO as a highly efficient GERS substrate 
for trace analyte detection. The spatially averaged ai-GERS spectra (solid lines) and their standard deviations (shaded backgrounds) across their respective probed regions ( $50 \mu \mathrm{m}^{2}, 75^{2}$ pixels) as a function of bulk R6G concentration (Figure 4.4(a)), show that upon decreasing the concentration, the Raman signature band amplitudes of $\mathrm{R} 6 \mathrm{G}$ and the fluorescence baseline also reduce. It is important to mention that R6G is a fluorescent molecule and conventional GERS measurements can be difficult to perform at these concentrations, especially when probing in air (lower SNR due to greater refractive index mismatch between air/R6G relative to water/R6G). However, the improvement in SNR offered by ai-GERS and the in-situ conditions of our measurement allow to detect $\mathrm{R} 6 \mathrm{G}$ on $\mathrm{rGO}$ at bulk concentrations as low as $5 \times 10^{-10} \mathrm{M}$ or $0.5 \mathrm{nM}$. Xinxin Yu et al. demonstrated Raman signatures of another fluorescent molecule, Rhodamine- $\mathrm{B}(\mathrm{RhB})$, on chemically reduced $\mathrm{GO}$ down to $5 \mathrm{x}$ $10^{-8} \mathrm{M}^{[23]}$. For comparison, we also report the ai-GERS spectrum of $5 \times 10^{-8} \mathrm{M}$ RhB-isothiocyanate on rGO (Figure 4.5).

The intensities of the characteristic Raman bands of R6G $\left(A_{610}, A_{772}\right.$ and $\left.A_{1649}\right)$, normalized by the silicon band area at $520 \mathrm{~cm}^{-1}\left(\mathrm{~A}_{\mathrm{Si}}\right)$, initially increase with the R6G concentration, and reach a plateau beyond $\sim 50 \mathrm{nM}$ (Figure 4.4(b)). For the low concentration regime (o-10 nM), the R6G intensities follow a linear trend with [R6G] (inset, Figure 4.4(b)). The plateauing at higher concentrations is attributed to the saturation of the R6G adsorption in the first layer in direct contact with the rGO substrate, from where the maximum Raman enhancement in GERS is generally believed to arise ${ }^{[7]}$. The evolution of the fluorescence baseline area $\left(A_{\text {Fluo }}\right)$ with [R6G] (Figure 4.4(c)) is consistent with this picture, suggesting that the fluorescence suppression by rGO is less efficient beyond the first contacting R6G layer. At the R6G concentrations where the Raman intensities have plateaued, the fluorescence intensities also tend to level off, albeit that a plateau is not reached. The ongoing increase of $A_{\text {Fluo }}$ at the highest $[\mathrm{R} 6 \mathrm{G}]$ could arise from the (increasingly abundant) free R6G molecules in bulk solution. Figure 4.4(d)-(e) show the corresponding 

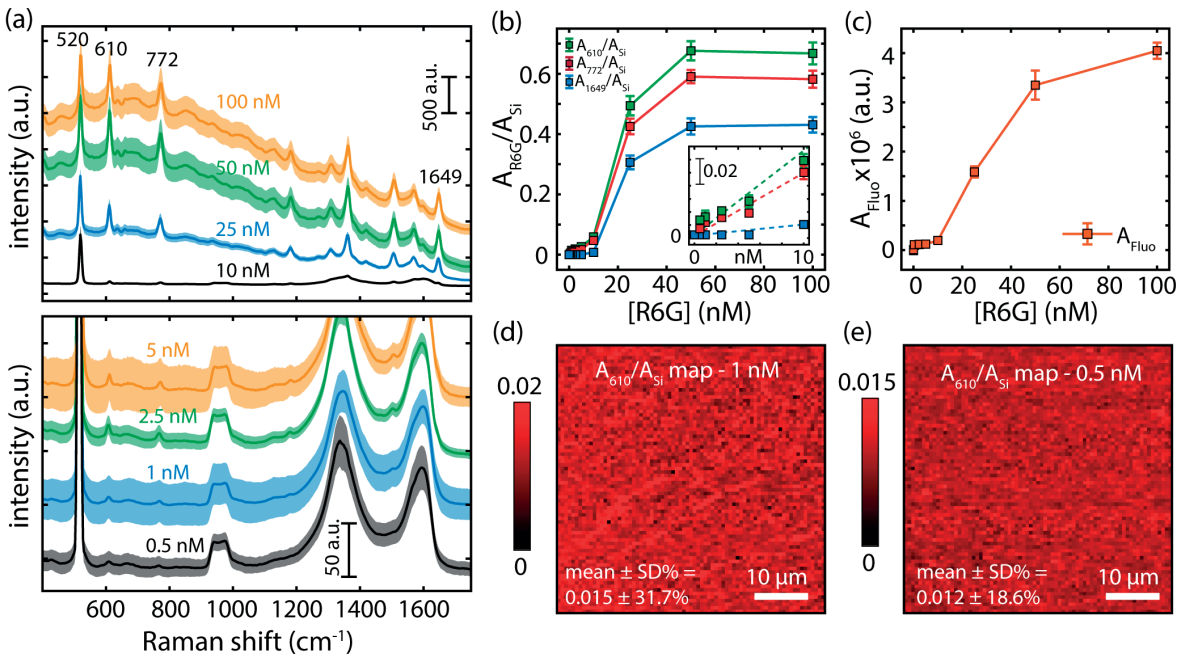

Figure 4.4: (a) Representative ai-GERS spectra (solid line - mean; shaded background standard deviation) on $r G O$ for a range of high (top panel) and low (bottom panel) $R 6 G$ concentrations. The spectra have been offset vertically for clarity. The LOD is determined to be $\approx 5 \times 10^{-10} M$. (b) Variation of the characteristic R6G Raman bands $\left(A_{610} / A_{S i}\right.$, $A_{772} / A_{S i}$ and $\left.A_{1649} / A_{S i}\right)$ and $(c)$ fluorescence baseline area $\left(A_{\text {Fluo }}\right)$ with $[R 6 G]$. The inset in (b) shows the linear variation (dashed lines) of the $R 6 G$ intensities for low concentrations ( 0 - $10 \mathrm{nM})$. (d-e) Corresponding Raman maps obtained by calculating the ratio of $A_{610}$ to $A_{S i}$ at $1 \times 10^{-9} \mathrm{M}$ and $5 \times 10^{-10} \mathrm{M}$, respectively. The ai-GERS facilitated Raman maps show uniform adsorption of $R 6 G$ on $r G O$ (relative standard deviations are indicated), even at $5 \times 10^{-10} \mathrm{M}$. 

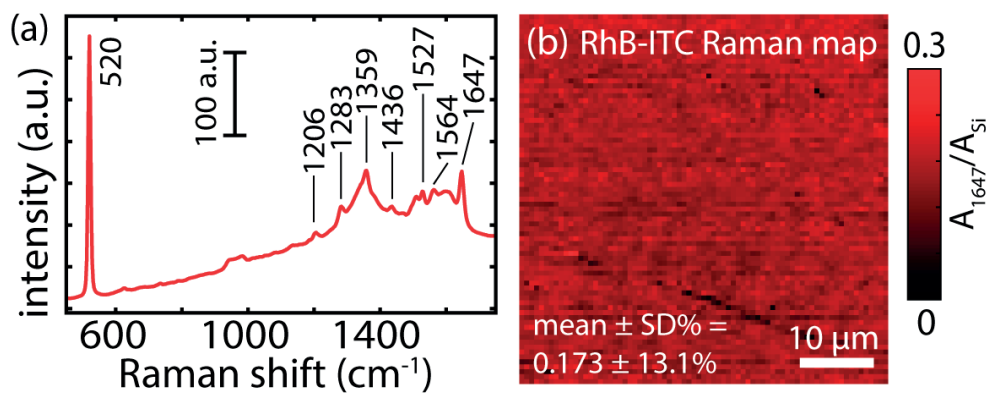

Figure 4.5: (a) Spatially averaged ai-GERS spectrum of RhB-ITC adsorbed onto $r G O$ $\left(I_{W}=0.5 \mathrm{~mW}\right)$ from a $50 \mathrm{nM}$ aqueous solution. The characteristic bands of RhB-ITC have been mentioned. (b) The corresponding spatial distribution of the adsorbed $R h B$ ITC, obtained by calculating the ratio of $A_{1647} / A_{S i}$. The mean and standard deviation of the intensity ratio across the probed region show the tendency of RhB-ITC to uniformly adsorb onto the photo-reduced GO flake.

Raman maps $\left(\mathrm{A}_{610} / \mathrm{A}_{\mathrm{Si}}\right)$ of the R6G distribution at $1 \times 10^{-9} \mathrm{M}$ and $5 \times 10^{-10} \mathrm{M}$, respectively. Raman maps at other probed concentrations are shown in Figure 4.6. The uniformity of the distribution (see relative standard deviations in Figure 4.4(d)-(e) and Figure 4.6) at all concentrations indicates the high affinity and homogeneous adsorption of the R6G molecules on these laser-reduced GO nano-sheets, underlining rGO's value as an efficient GERS substrate.

\subsubsection{Limit of detection on oxidized graphene}

Sung Huh et al. ${ }^{[13]}$ established a protocol for making UV/ozone-oxidized CVDgraphene substrates which give a ten-fold stronger Raman enhancement as compared to their unoxidized counterpart. In their work, CVD grown graphene samples supported on $\mathrm{SiO}_{2} / \mathrm{Si}$ substrate were subjected to UV-Ozone treatment for 5 minutes and directly used as GERS substrates. Following the same protocol, we use these oxidized graphene (oG) substrates to demonstrate the efficiency of ai-GERS for sub-nM R6G detection and imaging. The variation of the spatially averaged ( 50 


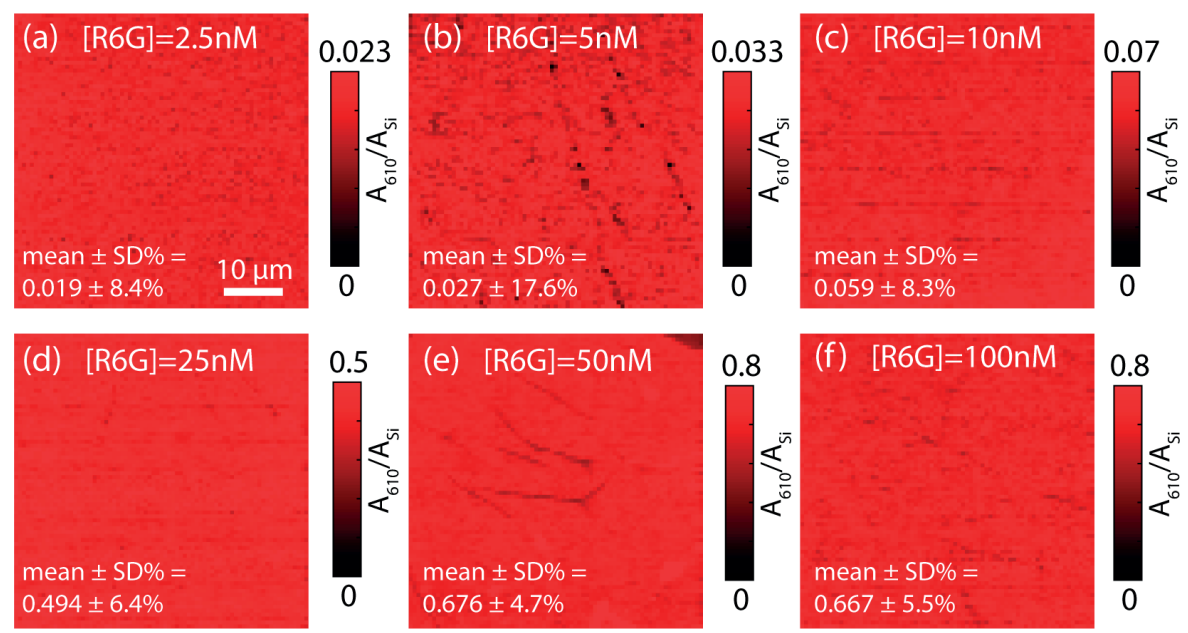

Figure 4.6: Raman maps of the spatial distribution of $R 6 G\left(A_{610} / A_{S i}\right)$, adsorbed onto $r G O\left(I_{W}=0.5 \mathrm{~mW}\right)$ from its bulk solutions at (a) $2.5 \mathrm{nM},($ b) $5 \mathrm{nM},($ c) $10 \mathrm{nM},(d) 25$ $n M,(e)$ so $n M$ and $(f) 100 \mathrm{nM}$. The mean and standard deviation of the intensity ratios are mentioned. Scale bar: $10 \mu \mathrm{m}$. 
$\mu \mathrm{m}^{2}, 75^{2}$ pixels) ai-GERS spectra vs. the bulk [R6G] on oG substrates (Figure 4.7) show that the characteristic R6G bands can be detected for concentrations as low as $5 \times 10^{-10} \mathrm{M}$, lower than any previously reported LOD for this system. Recently, Donghua Liu et al. detected R6G layers dried onto ultra-clean graphene quantum dots (GQD) substrates from a solution at $1 \times 10^{-9} \mathrm{M}^{[16]}$. Note that, while the absolute LOD is thus reduced by a factor of 2 , our improvement is also achieved using commercially available materials with minimal preparation effort. Furthermore, our in-situ measurements are performed while the substrate remains in contact with a fluid of fixed specified concentration, thereby avoiding possible up-concentration effects due to drying (leading to an overestimation of the actual LOD; see Figure 4.8 for the Raman spectrum of a dried $1 \times 10^{-11} \mathrm{M}$ R6G droplet on $\mathrm{oG}$ ).

Quantification of the R6G band intensities as a function of [R6G] (Figure 4.7(b)) displays a linear trend throughout the probed concentration range, in contrast to rGO where such linearity was only observed for lower concentrations (inset, Figure $4.4(\mathrm{~b}))$. Following the suggestion of Xi Ling et al. ${ }^{[1]}$, who reported sub-monolayer adsorption for bulk concentrations below $8 \times 10^{-7} \mathrm{M}$, we attribute the linear behavior in Figure 4.7(b) to incomplete adsorption within the first monolayer (for the investigated concentration range). It also turns out that the $A_{R 6 G} / A_{S i}$ band ratios are significantly lower on oG as compared to the $\mathrm{rGO}$ case. The evolution of the fluorescence area with [R6G] on oG, however, behaves similarly to the rGO case (compare Figure 4.7(c) with Figure 4.4(c)). Within the (diffraction limited) spatial resolution, Figures $4.7(\mathrm{~d})$ and (e) further support the picture of partial coverage on the oG substrates at bulk R6G concentrations of $1 \times 10^{-9} \mathrm{M}$ and $5 \times 10^{-10} \mathrm{M}$ respectively (for Raman maps at other R6G concentrations, refer Figure 4.9). ai-GERS-facilitated Raman imaging thus provides unprecedented insights into sub-monolayer adsorption. 
(a)
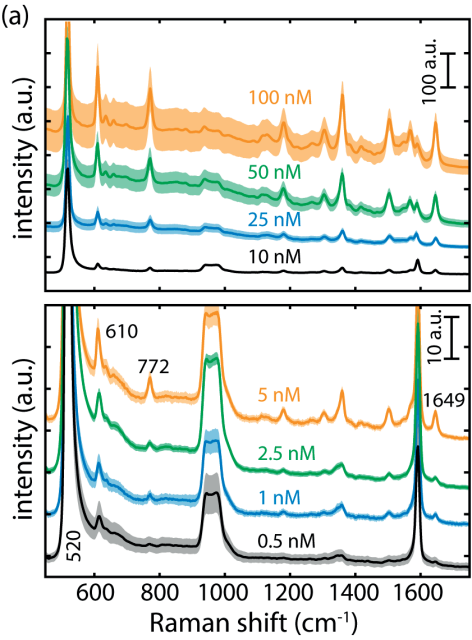

(b)

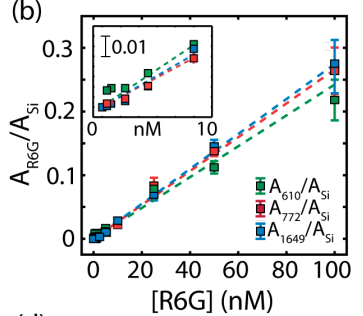

(d)

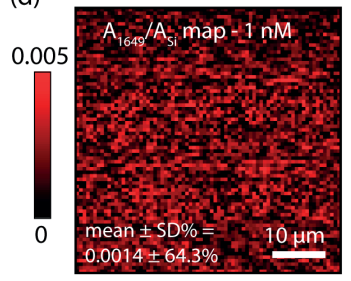

(c)

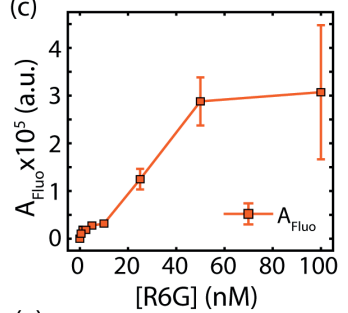

(e)

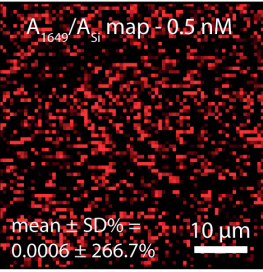

Figure 4.7: (a) Representative ai-GERS spectra (solid line - mean, shaded background standard deviation) of $R 6 G$ on oxidized-graphene (oG) substrates for a range of high (top panel) and low (bottom panel) R6G concentrations. The spectra have been offset vertically for clarity. The experimental LOD is $\approx 5 \times 10^{-10} \mathrm{M}$. (b) Variation of the characteristic R6G Raman bands $\left(A_{610} / A_{S i}, A_{772} / A_{S i}\right.$ and $\left.A_{1649} / A_{S i}\right)$ and (c) fluorescence background $\left(A_{\text {Fluo }}\right)$ with $[R 6 G]$. The inset in (b) shows the variation of the R6G intensities for low concentrations (o - $10 \mathrm{nM}$ ). (d-e) Corresponding Raman maps obtained by calculating $A_{1649} / A_{S i}$ at $1 \times 10^{-9} M$ and $5 \times 10^{-10} M$, respectively. The ai-GERS-facilitated Raman maps help to visualize sub-monolayer coverage of R6G on oG for these low bulk concentrations. 

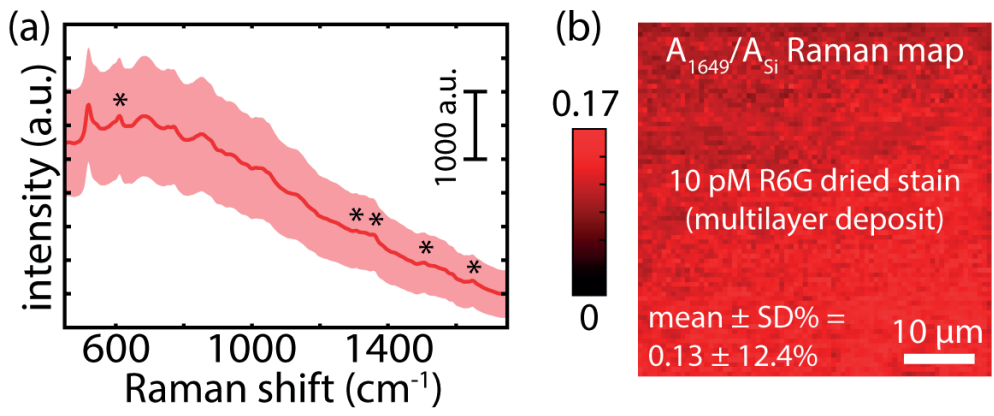

Figure 4.8: (a) Spatially averaged ai-GERS spectrum of a 10 pM R6G $\operatorname{droplet}(10 \mu \mathrm{L})$ dried onto $o G$. The substrate containing the dried deposit was then immersed in an ambient 10 pM R6G solution and the ai-GERS measurement was made in-situ. The intense fluorescence baseline is due to the accumulation of R6G multilayers in the form of a "coffee-stain" after drying. The characteristic R6G bands have been marked with asterisks. ( $b$ ) The corresponding spatial distribution of $R 6 G$, obtained by calculating the ratio of $A_{1649} / A_{S i}$. The mean value of the $A_{1649} / A_{S i}$ intensity ratio is comparable to the in-situ ai-GERS case at 50 $n M$ R6G (Figure 4.7(b)), highlighting the overestimation of the LOD that can be caused when probing dried layers in air. 

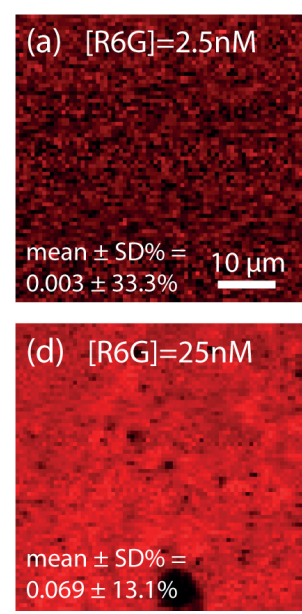
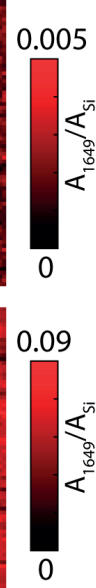
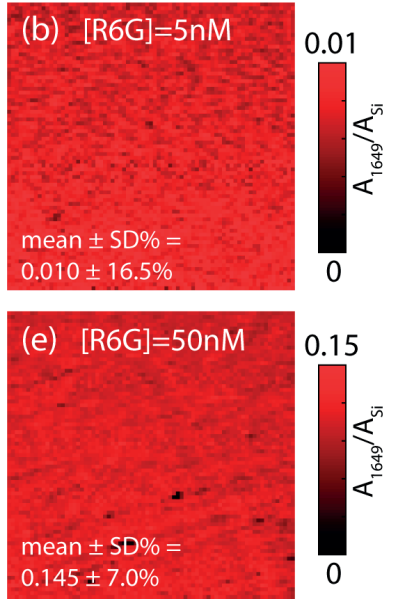
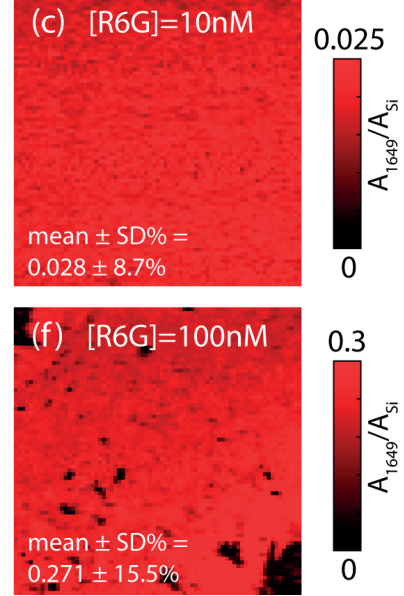

Figure 4.9: Raman maps of the spatial distribution of $R 6 G\left(A_{1649} / A_{S i}\right)$, adsorbed onto oxidized-graphene (oG) substrates from bulk solutions with concentrations of (a) $2.5 \mathrm{nM}$, (b) $5 \mathrm{nM},(c) 10 \mathrm{nM},(d) 25 \mathrm{nM}$, (e) $50 \mathrm{nM}$ and (f) $100 \mathrm{nM}$. The mean and standard deviation of the intensity ratios are mentioned. Scale bar: $10 \mu \mathrm{m}$.

\subsubsection{Applications of in-situ ai-GERS}

The in-situ nature of the measurement allows us to directly probe the adsorption kinetics of R6G on single-flake rGO (in contrast to GERS studies that examine dried analyte layers in air). Dynamic xy-Raman imaging ( $50 \times 50 \mu \mathrm{m}^{2}$, 50 x 50 pixels, $I_{P}=0.25 \mathrm{~mW}$ and $t_{P}=0.05 \mathrm{~s} /$ pixel) for $175 \mathrm{~min}$ shows the gradual R6G adsorption onto $\mathrm{rGO}$ (created with $\mathrm{I}_{\mathrm{W}}=0.5 \mathrm{~mW}$ ) upon exposure to a $50 \mathrm{nM}$ solution. The spatially averaged spectrum displays an increase in the R6G fingerprint band amplitudes (Figure 4.10(a)), leading to an S-shaped "adsorption curve" of $\mathrm{A}_{610} / \mathrm{A}_{\mathrm{Si}}$ versus time (Figure 4.10(b)). A steady state appears to be reached after $\sim 2$ hours. The corresponding time evolution of the Raman maps is shown in Figure 4.11.

As another application, we analyzed the presence of trace amounts of R6G that had been added to a commercial fruit juice. Xanthene-based dyes like Rhodamine are 
(a)
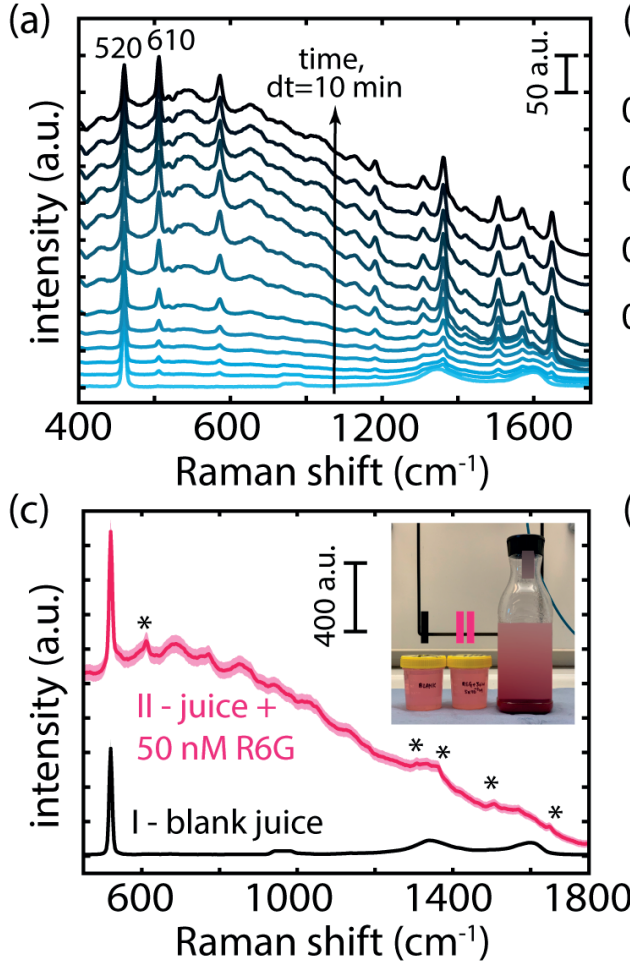

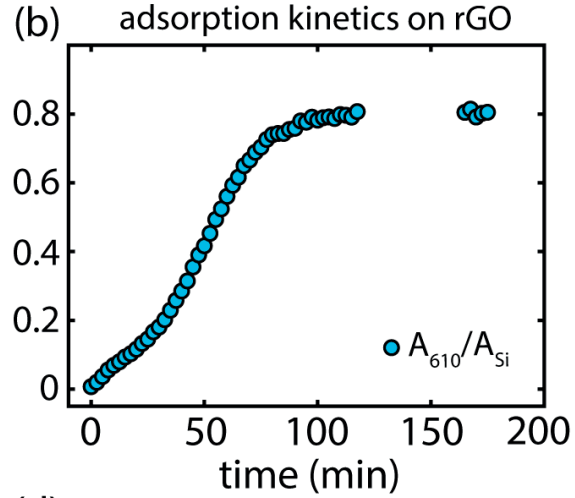

(d)

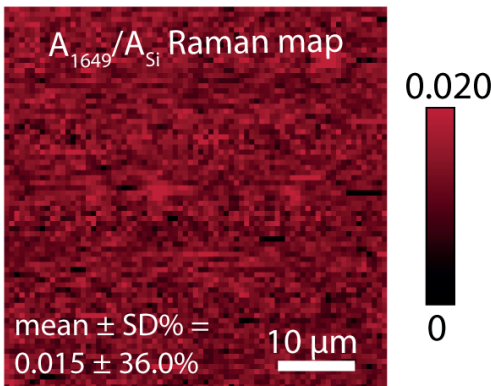

rGO for detecting adulteration

in commercial juice

Figure 4.10: (a) Evolution of the spatially averaged ai-GERS spectrum of R6G adsorbing onto $r G O$ after $50 \mathrm{nM} R 6 \mathrm{G}$ addition at $t=0 \mathrm{~min}$. The spectra have been offset vertically for clarity. (b) Adsorption curve depicting the growth of the $A_{610} / A_{S i}$ ratio over time. A steady state is reached after $\sim 2$ hours. (c) Comparison of the ai-GERS response of an original juice vs. one that was adulterated with $50 \mathrm{nM}$ of R6G, on $r G O$. Inset shows the photograph of the juice and the two solutions: I - blank and II - adulterated juice. (d) Raman map of $A_{1649} / A_{S i}$, reflecting the $R 6 G$ adsorbed from the adulterated juice on $r G O$. 

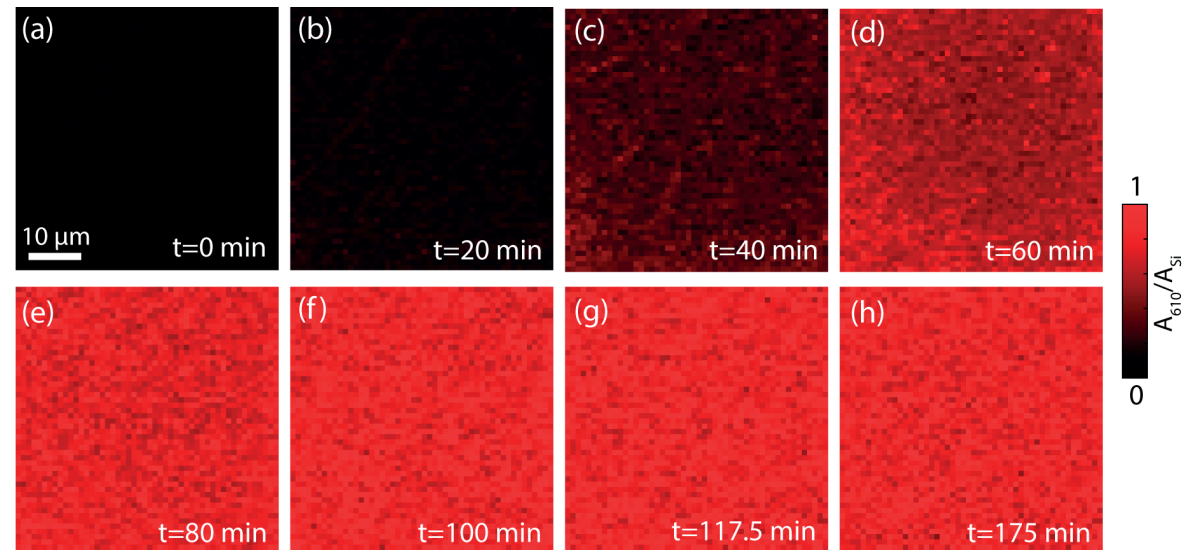

Figure 4.11: (a)-(h) Evolution of the spatial R6G distribution $\left(A_{610} / A_{S i}\right)$ on a rGO flake $\left(I_{W}=0.5 \mathrm{~mW}\right)$ after addition of $50 \mathrm{nM} \mathrm{R6G}($ at $t=0 \mathrm{~min})$. The coverage and intensity ratios increase with time and reaches equilibrium after $\sim 120 \mathrm{~min}$. The mean intensity ratio per time slice $(2.5 \mathrm{~min})$ is extracted and plotted as an adsorption kinetics curve in Figure $4.10(b)$. All Raman maps have been plotted against the same colour scale. Scale bar: $10 \mu \mathrm{m}$. 
widely used in the textile and leather industries as colorants, but have been shown to be carcinogenic and cytotoxic ${ }^{[35]}$. In addition to the harmful health effects when reaching drinking water ${ }^{[36]}$, there are also growing concerns about Rhodamine dyes being used as illegal food colorants ${ }^{[37,38]}$. Here, we demonstrate the ability of in-situ ai-GERS to detect and visualize $50 \mathrm{nM}$ of R6G dissolved in an as-purchased fruit juice (inset, Figure $4.10(\mathrm{c})$ ). To this end, we utilize $\mathrm{rGO}\left(\mathrm{I}_{\mathrm{W}}=0.5 \mathrm{~mW}\right)$ as a GERS substrate, and compare the Raman responses of the original (i.e. blank) and adulterated juices. The blank juice (Figure 4.10(c), black curve) shows no particular Raman features except for the Si and rGO bands from the substrate, whereas the adulterated one (magenta curve) clearly shows a fluorescence baseline and characteristic R6G bands (denoted by $\left.{ }^{*}\right)$. The Raman map of the adsorbed R6G $\left(A_{1649} / A_{S i}\right)$ from the adulterated juice is shown in Figure 4.10(d). These results clearly highlight the capabilities of in-situ ai-GERS for detecting trace analytes in complex solutions. Both the high affinity of the conjugated R6G molecule to adsorb onto the rGO lattice and the algorithm-improved SNR enhancement of Raman signals are pertinent to the ultra-sensitive detection of analytes on graphene oxide analogues.

\subsection{Conclusion}

In summary, we utilized an algorithm-improved (ai-) mode of Raman imaging [33] to considerably improve the sensitivity of analyte detection in the field of grapheneenhanced Raman spectroscopy (GERS). We demonstrated that reduced graphene oxide (rGO), obtained via laser writing, is a far superior GERS substrate as compared to its unreduced counterpart in terms of fluorescence suppression and Raman enhancement. Our results show the capability of ai-GERS in improving the limit of detection (LOD) of rhodamine-6g layers adsorbed onto single rGO flakes, to bulk R6G concentrations as low as $5 \times 10^{-10} \mathrm{M}$. Keeping in line with the practical necessity of avoiding complex substrate fabrication for sensitive analyte sensing, we also report an improved LOD of $5 \times 10^{-10} \mathrm{M}$ for R6G on facile oxidized graphene (oG) 
samples. ai-GERS facilitated imaging provides direct and non-invasive visualization of the adsorbed analyte distribution, even at sub-nanomolar concentrations. From a practical standpoint, we show the advantage of in-situ ai-GERS measurements to monitor and image the adsorption kinetics of R6G on rGO, and also show the capability of rGO as a sensor for detecting trace adulteration in a commercial juice. We believe that ai-GERS could potentially improve low concentration analyte detection, quantification and imaging on various substrates useful for chemical and bio-sensing applications ${ }^{[30]}$.

\section{Bibliography}

[1] X. Ling, L. Xie, Y. Fang, H. Xu, H. Zhang, J. Kong, M. S. Dresselhaus, J. Zhang, and Z. Liu, "Can graphene be used as a substrate for Raman enhancement?," Nano letters, vol. 10, no. 2, pp. 553-561, 2010.

[2] M. Fleischmann, P.J.Hendra, and A.J. McQuillan, "Raman spectra of pyridine adsorbed at a silver electrode," Chemical physics letters, vol. 26, no. 2, pp. $163-$ $166,1974$.

[3] A. Campion and P. Kambhampati, "Surface-enhanced Raman scattering," Chemical society reviews, vol. 27, no. 4, pp. 241-250, 1998.

[4] X. Ling, S. Huang, S. Deng, N. Mao, J. Kong, M. S. Dresselhaus, and J. Zhang, "Lighting up the Raman signal of molecules in the vicinity of graphene related materials," Accounts of chemical research, vol. 48, no. 7, pp. 1862-1870, 2015.

[5] L. Xie, X. Ling, Y. Fang, J. Zhang, and Z. Liu, "Graphene as a substrate to suppress fluorescence in resonance Raman spectroscopy," Journal of the American Chemical Society, vol. 131, no. 29, pp. 9890-9891, 2009.

[6] W. Xu, N. Mao, and J. Zhang, "Graphene: a platform for surface-enhanced Raman spectroscopy,” Small, vol. 9, no. 8, pp. 1206-1224, 2013. 
[7] X. Ling and J. Zhang, "First-layer effect in graphene-enhanced Raman scattering," Small, vol. 6, no. 18, pp. 2020-2025, 2010.

[8] K. Tantiwanichapan, X. Wang, H. Durmaz, Y. Li, A. K. Swan, and R. Paiella, "Graphene terahertz plasmons: A combined transmission spectroscopy and Raman microscopy study," ACS Photonics, vol. 4, no. 8, pp. 2011-2017, 2017.

[9] Y. Zhao, Y. Xie, Z. Bao, Y. H. Tsang, L. Xie, and Y. Chai, "Enhanced SERS stability of r6g molecules with monolayer graphene," The Journal of Physical Chemistry C, vol. 118, no. 22, pp. 11827-11832, 2014.

[10] X.-M. Lin, Y. Cui, Y.-H. Xu, B. Ren, and Z.-Q. Tian, "Surface-enhanced Raman spectroscopy: substrate-related issues," Analytical and bioanalytical chemistry, vol. 394, no. 7, pp. 1729-1745, 2009.

[11] H. Xu, L. Xie, H. Zhang, and J. Zhang, "Effect of graphene fermi level on the Raman scattering intensity of molecules on graphene," ACS nano, vol. 5, no. 7, pp. 5338-5344, 2011.

[12] Q. Hao, S. M. Morton, B. Wang, Y. Zhao, L. Jensen, and T. Jun Huang, "Tuning surface-enhanced Raman scattering from graphene substrates using the electric field effect and chemical doping," Applied physics letters, vol. 102, no. 1, p. $011102,2013$.

[13] S. Huh, J. Park, Y. S. Kim, K. S. Kim, B. H. Hong, and J.-M. Nam, "UV/Ozoneoxidized large-scale graphene platform with large chemical enhancement in surface-enhanced Raman scattering," ACS nano, vol. 5, no. 12, pp. 9799-9806, 2011.

[14] S. Feng, M. C. dos Santos, B. R. Carvalho, R. Lv, Q. Li, K. Fujisawa, A. L. Elías, Y. Lei, N. Perea-López, and M. Endo, "Ultra-sensitive molecular sensor using N-doped graphene through enhanced Raman scattering," Science advances, vol. 2, no. 7, p. e1600322, 2016. 
[15] V. Valeš, K. Drogowska-Horná, V. L. Guerra, and M. Kalbáč, "Grapheneenhanced Raman scattering on single layer and bilayers of pristine and hydrogenated graphene," Scientific reports, vol. 10, no. 1, pp. 1-9, 2020.

[16] D. Liu, X. Chen, Y. Hu, T. Sun, Z. Song, Y. Zheng, Y. Cao, Z. Cai, M. Cao, and L. Peng, "Raman enhancement on ultra-clean graphene quantum dots produced by quasi-equilibrium plasma-enhanced chemical vapor deposition," Nature communications, vol. 9, no. 1, pp. 1-10, 2018.

[17] S. Huang, X. Ling, L. Liang, Y. Song, W. Fang, J. Zhang, J. Kong, V. Meunier, and M. S. Dresselhaus, "Molecular selectivity of graphene-enhanced Raman scattering," Nano letters, vol. 15, no. 5, pp. 2892-2901, 2015.

[18] S. Sil, N. Kuhar, S. Acharya, and S. Umapathy, "Is chemically synthesized graphene 'really' a unique substrate for SERS and fluorescence quenching?", Scientific reports, vol. 3, p. 3336, 2013.

[19] H. Yang, H. Hu, Y. Wang, and T. Yu, "Rapid and non-destructive identification of graphene oxide thickness using white light contrast spectroscopy," Carbon, vol. 52, pp. 528-534, 2013.

[20] K. Krishnamoorthy, M. Veerapandian, K. Yun, and S.-J. Kim, "The chemical and structural analysis of graphene oxide with different degrees of oxidation," Carbon, vol. 53, pp. 38-49, 2013.

[21] S. Pei and H.-M. Cheng, "The reduction of graphene oxide," Carbon, vol. 50, no. 9, pp. $3210-3228,2012$.

[22] Z. Wei, D. Wang, S. Kim, S.-Y. Kim, Y.Hu, M. K. Yakes, A. R. Laracuente, Z. Dai, S. R. Marder, and C. Berger, "Nanoscale tunable reduction of graphene oxide for graphene electronics," Science, vol. 328, no. 5984, pp. 1373-1376, 2010. 
[23] X. Yu, H. Cai, W. Zhang, X. Li, N. Pan, Y. Luo, X. Wang, and J. Hou, "Tuning chemical enhancement of SERS by controlling the chemical reduction of graphene oxide nanosheets," ACS nano, vol. 5, no. 2, pp. 952-958, 2011.

[24] L. Wang, Y. Zhang, Y. Yang, and J. Zhang, "Strong dependence of surface enhanced Raman scattering on structure of graphene oxide film," Materials, vol. 11 , no. 7, p. $1199,2018$.

[25] C. K. Chua and M. Pumera, "Chemical reduction of graphene oxide: a synthetic chemistry viewpoint," Chemical Society Reviews, vol. 43, no. 1, pp. 291312,2014 .

[26] P. Zhang, Z. Li, S. Zhang, and G. Shao, "Recent advances in effective reduction of graphene oxide for highly improved performance toward electrochemical energy storage," Energy and Environmental Materials, vol. 1, no. 1, pp. 5-12, 2018 .

[27] Y. Zhang, L. Guo, H. Xia, Q. Chen, J. Feng, and H. Sun, "Photoreduction of graphene oxides: methods, properties, and applications," Advanced Optical Materials, vol. 2, no. 1, pp. 10-28, 2014.

[28] R. Arul, R. N. Oosterbeek, J. Robertson, G. Xu, J. Jin, and M. C. Simpson, "The mechanism of direct laser writing of graphene features into graphene oxide films involves photoreduction and thermally assisted structural rearrangement," Carbon, vol. 99, pp. 423-431, 2016.

[29] B. Ma, R. D. Rodriguez, A. Ruban, S. Pavlov, and E. Sheremet, "The correlation between electrical conductivity and second-order Raman modes of laserreduced graphene oxide," Physical Chemistry Chemical Physics, vol. 21, no. 19, pp. 10125-10134, 2019.

[30] Z. Wang, S. Wu, L. C. Ciacchi, and G. Wei, "Graphene-based nanoplatforms 
for surface-enhanced Raman scattering sensing," Analyst, vol. 143, no. 21, pp. 5074-5089, 2018.

[3 1 ] A. Silver, H. Kitadai, H. Liu, T. Granzier-Nakajima, M. Terrones, X. Ling, and S. Huang, "Chemical and bio-sensing using graphene-enhanced Raman spectroscopy," Nanomaterials, vol. 9, no. 4, p. 516, 2019.

[32] J. S. Mehta, A. C. Faucett, A. Sharma, and J. M. Mativetsky, "How reliable are Raman spectroscopy measurements of graphene oxide?," The Journal of Physical Chemistry C, vol. 121, no. 30, pp. 16584-16591, 2017.

[33] S. Nair, J. Gao, Q. Yao, M. H. Duits, C. Otto, and F. Mugele, "Algorithmimproved high speed and non-invasive confocal Raman imaging of twodimensional materials," National Science Review, 2019.

[34] H. Abdi and L. J. Williams, "Principal component analysis," Wiley interdisciplinary reviews: computational statistics, vol. 2, no. 4, pp. 433-459, 2010.

[35] E. R. Nestmann, G. R. Douglas, T. I. Matula, C. E. Grant, and D. J. Kowbel, "Mutagenic activity of rhodamine dyes and their impurities as detected by mutation induction in salmonella and DNA damage in chinese hamster ovary cells," Cancer Research, vol. 39, no. 11, pp. 4412-4417, 1979.

[36] R. Jain, M. Mathur, S. Sikarwar, and A. Mittal, "Removal of the hazardous dye rhodamine-B through photocatalytic and adsorption treatments," Journal of Environmental Management, vol. 85, no. 4, pp. 956-964, 2007.

[37] P. Qi, Z. Lin, J. Li, C. Wang, W. Meng, H. Hong, and X. Zhang, “Development of a rapid, simple and sensitive HPLC-FLD method for determination of rhodamine B in chili-containing products," Food chemistry, vol. 164, pp. 98-103, 2014. 
[38] H. Tian, N. Zhang, L. Tong, and J. Zhang, "In situ quantitative graphene-based surface-enhanced Raman spectroscopy," Small Methods, vol. 1, no. 6, p. $1700126,2017$. 
"A drop of water, if it could write out its own history, would explain the universe to us."

Lucy Larcom

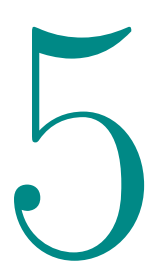

In-situ observation of reactive wettability alteration using algorithm-improved confocal Raman microscopy ${ }^{\dagger}$

The WetTABILITy of complex fluids on surfaces usually depends on the adsorption of solutes to any of the constituting interfaces. Controlling such interfacial processes by varying the composition of a phase enables the design of smart responsive systems.

\footnotetext{
${ }^{+}$Published as: Nair, Sachin et al., Journal of colloid and interface science 584 (2021): 551560.
} 
Our goal is to demonstrate that $3 \mathrm{D}$ Confocal Raman Microscopy (CRM) can reveal the mechanistic details of such processes by allowing to simultaneously monitor the contact angle variation and redistribution of the chemical species involved. Motivated by the enhanced oil recovery process of low salinity water flooding, we studied the response of picolitre oil drops on mineral substrates upon varying the ambient brine salinity. The substrates were pre-coated with thin layers of deuterated-stearic acid (surfactant) that display salinity-dependent stability. $3 \mathrm{D}$ CRM imaging using a recently proposed faster 'ai' (algorithm-improved) mode reveals that the surfactant layer is stable at high salinities, leading to preferential oil wetting. Upon reducing the ambient brine salinity, this layer decomposes and the investigated surfaces of mica and - somewhat less pronounced - silica become more water wet. Eventually, the surfactant is found to partly dissolve in the oil and partly precipitate at the oil-water interface. We anticipate that ai-3D-CRM will prove useful to holistically study similar systems displaying reactive wetting.

\subsection{Introduction}

When a surface is reactive to a contacting liquid or responsive to an external stimulus, its wettability is also tuned by the reaction ${ }^{[1]}$. This behavior can be utilized to design surfaces with smart wettability ${ }^{[2,3]}$, with potential use in self-cleaning surfaces ${ }^{[4-6]}$, microfluidics ${ }^{[7]}$, functional membranes ${ }^{[8,9]}$ and pickering emulsions ${ }^{[10]}$. The phenomenon of reactive wetting is not only pertinent to artificially tailored smart materials, but it also occurs in geological processes. For example, soils often become very hydrophobic during longer periods of dry weather, preventing efficient water absorption and retention upon subsequent rainfall. Organic species on mineral surfaces can adsorb and desorb and change their configuration depending on ambient humidity, rendering the initially hydrophilic mineral hydrophobic ${ }^{[11]}$. Certain parameters like $\mathrm{pH}$ and the presence of divalent cations can also affect the soil water repellency persistence $^{[12]}$. A reactive wettability alteration in the opposite direction has been ob- 
served in enhanced oil recovery (EOR), where rock minerals become more hydrophilic after exposure to brines of lower salinity. In low salinity EOR, this change in wettability is believed to be the main cause of the enhanced oil release ${ }^{[13]}$. However, our understanding of such wettability alterations is often incomplete. This is partly due to a lack of techniques that can address the appropriate range of length scales in one experiment. Microscopic characterization of reactive wettability is therefore of key importance for rational engineering of smart wetting applications.

Wettability characterization is typically performed by taking optical images of a sessile droplet on a substrate and then extracting the contact angles at the three phase contact line (TPCL). However, this method faces two challenges for characterizing reactive wettability alteration: it often limits the characterization to the millimeter scale and lacks any chemical specificity. Many practical applications such as droplet condensation, microfluidics and enhanced oil recovery, involve the wettability of micron-sized droplets. Furthermore, most practical substrates are nonhomogeneous, causing the wettability to be sensitive to size of the substrate features. The micron-scale wettability could therefore be different from its millimeter-scale counterpart.

To address this problem, various microscopic techniques have been employed, such as atomic force microscopy $(\mathrm{AFM})^{[14]}$, interference microscopy ${ }^{[15]}$, $\mathrm{x}$-ray tomography ${ }^{[16,17]}$ and confocal laser scanning microscopy ${ }^{[18-20]}$, enabling wettability characterization even down to the nano-scale ${ }^{[14]}$. However, none of these methods provide chemical specificity, which is crucial for revealing the mechanistic details of the wettability alteration. For example, surfactant induced autophobing ${ }^{[21-24]}$ is a complex phenomenon, because the dynamics involve multiple chemical components and interfaces. In the absence of a detailed information on which materials get redistributed and how, mechanistic interpretations and modelling have to rely on assumptions or ex-situ characterizations. While (fluorescent) dyes can be used to label the different fluid phases and sometimes also the surfactant, these components 
have a tendency to adsorb at interfaces, thereby affecting the physical chemistry of the system. Similar problems of insufficient information on the (dynamic) presence and chemical nature of compounds are also prevalent in other systems like emulsions, biological cells, etc. Some of these additionally show heterogeneity at micron length scales, which further aggravates the issue.

Confocal Raman microscopy (CRM), on the contrary, has the potential to address these problems. Its vibrational spectroscopic nature provides unique access to the chemical information of every probed component, and its confocal nature provides three dimensional spatial resolution down to the sub-micron scale. Therefore, it could be used to characterize both the chemical reaction on the substrate and the resultant wettability alteration, giving us an opportunity to study the system more holistically. Until recently, the slowness of confocal Raman mapping was a significant hurdle. Due to the inherently low Raman scattering efficiency, measuring a spectrum from a single laser spot would normally take $\mathrm{O}(100 \mathrm{~ms})$ of exposure time. Scanning the laser over a typical range of $\mathrm{O}(10 \mu \mathrm{m})$ would then require $\mathrm{O}(1 \mathrm{~h})$ in $2 \mathrm{D}$, and $\mathrm{O}(1$ day $)$ in ${ }_{3} \mathrm{D}$. Such reaction time scales are often impractical for reactive wettability characterization. This problem can however be mitigated via the recently developed algorithm-improved confocal Raman microscopy (ai-CRM) [25], which is capable of increasing the scanning speed by 1-2 orders of magnitude by making use of principal component analysis-based denoising. The improvement in signal-to-noise ratio (SNR) of the spectral data offered by ai-CRM facilitates fast, simultaneous volumetric mapping of multiple components in-situ at the micron-scale, provided that the number of (spectrally overlapping) components is not too large and the local concentrations are not too low.

In this chapter we use ai-CRM to elucidate some mechanistic aspects of a chemically triggered wettability alteration. We consider a system of mica/silica, oil and brine that is often used as a model to represent the low salinity water flooding (LSWF) process in enhanced oil recovery. LSWF is an environmentally friendly and cost- 
effective EOR technique. In favorable cases (depending on the oil field) a simple dilution of the flooding water can increase the oil recovery efficiency by up to ten percent ${ }^{[26]}$. However, a wide deployment of LSWF is still hindered by a variability in the outcomes, and debates about the microscopic mechanism. Many mechanisms have been proposed ${ }^{[13,27,28,26,29]}$ including $\mathrm{pH}$-increase ${ }^{[30]}$, fines migration ${ }^{[31,32]}$, double layer expansion ${ }^{[33]}$, and multi-ion exchange (MIE) ${ }^{[34]}$. The MIE mechanism states that divalent cations in the high-salinity formation brine can adsorb on the negatively charged mineral substrate (typically quartz and clay in sandstone reservoirs), thereby forming a bridge between the substrate and negatively charged surfactants in the crude oil. As a result, the mineral substrate is turned hydrophobic. Injecting low salinity water weakens the cation bridge, supposedly leading to a partial or complete release of the surfactant. As a consequence, the mineral surface becomes more hydrophilic, and oil displacement more efficient. In recent years, the MIE mechanism has been receiving increasing interest, and several aspects of it have been confirmed for model Crude Oil/Brine/ Rock (COBR) systems. For example, using mica and silica substrates, fatty-acid laden decane, and brines at varying ion content, (ex-situ) AFM studies confirmed the adsorption of divalent cations, as well as the subsequent adsorption of surfactants on the mineral substrate ${ }^{[35]}$. Contact angle goniometry confirmed the resultant wettability changes on the macroscopic scale ${ }^{[36]}$. However, to the best of our knowledge, there exists no direct experimental proof in literature which directly links the desorption of the surfactant to the wettability alteration.

In this paper, we use ai-CRM to provide direct evidence of this reactive wettability alteration in response to lowered ambient salinity. We illustrate this at the microscopic scale, which is the scale relevant to real oil reservoir conditions. Using mica ('clay') and glass ('rock') as model substrates, we first apply a coating of a surfactant and then immerse them in ambient brine. Subsequent deposition of oil leads to the formation of a microscopic droplet, whose shape is imaged in ${ }_{3} \mathrm{D}$ with the con- 
focal Raman microscope. Using a deuterated surfactant, we simultaneously access the distributions of the separate components: surfactant, oil, water and substrate. Inspecting the changes after lowering the salinity of the ambient brine then allows us to correlate the contact angle change to the redistribution of the surfactant.

\subsection{Experimental section}

\section{Materials}

All chemicals were reagent grade and purchased from Merck, except for mineral oil (mixture of liquid hydrocarbons, density $=0.84 \mathrm{~g} \mathrm{~mL}^{-1}$, BioReagent light oil), which was purchased from Sigma-Aldrich. As substrates, mica sheets (B \& M Mica Co., Inc.) and silicon wafers (Okmetic, P type wafers) with $30 \mathrm{~nm}$ of thermally grown $\mathrm{SiO}_{2}$ on top were used. Mica substrates were cleaved with Scotch tape right before the dip-coating procedure. $30 \mathrm{~nm}-\mathrm{SiO}_{2} / \mathrm{Si}$ wafers were cleaned by sonicating them in an ethanol and iso-propanol 1:1 mixture and then washing with deionized water. Then they were dried with a nitrogen gun and put in a UV/ozone cleaner (BioForce Nanosciences, Inc.) for 15 minutes just before dip-coating.

The brine phase for dip coating contained a $10 \mathrm{mM} \mathrm{CaCl}_{2}$ and $2 \mathrm{mM} \mathrm{NaHCO}_{3}$ solution in deionized water (Millipore, resistivity $18.2 \mathrm{M} \Omega \mathrm{cm}$ ), similar to their concentrations in seawater. The $\mathrm{pH}$ was brought to 8 by adding small amounts of 0.1 $\mathrm{N} \mathrm{NaOH}$. The oil phase for the surfactant deposition was prepared by dissolving deuterated-stearic acid $\left(\mathrm{C}_{18} \mathrm{D}_{36} \mathrm{O}_{2}, \mathrm{~d}\right.$-SA) in n-decane (filtered 5 times through a $5 \mathrm{~cm}$ column of aluminum oxide powder prior to adding the d-SA), such that the concentration was $2 \mathrm{mM}$. The reason for choosing the deuterated variety was to prevent overlap of the $\mathrm{C}_{18} \mathrm{H}_{36} \mathrm{O}_{2}$ Raman band with the mineral oil band, both of which show an intense $\mathrm{CH}_{2}$ stretching mode between 2800 and $3000 \mathrm{~cm}^{-1}$. Deuterating the stearic acid shifts the band to $2000-2300 \mathrm{~cm}^{-1}$. The Raman spectrum of each individual component of the system is shown in Figure 5.1, which highlights the ex- 


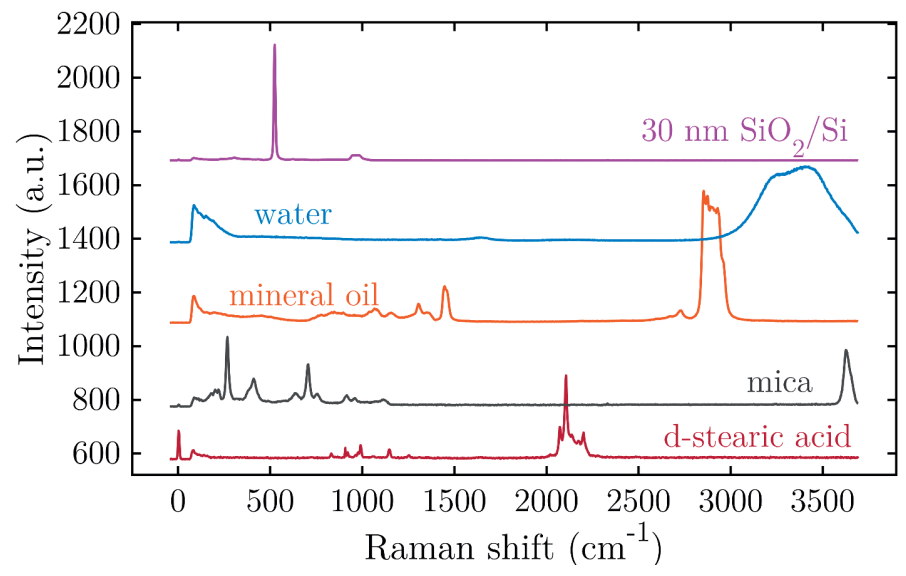

Figure 5.1: Individual Raman spectrum of all the components involved in the study. Differences in the probed band position gives excellent spectral contrast and readily enables distinction between any two components.

cellent spectral contrast between the different probed bands used for $3 \mathrm{D}$ Raman imaging. Artificial sea water (ASW) was prepared to contain $485 \mathrm{mM}$ of $\mathrm{NaCl}, 10.3 \mathrm{mM}$ of $\mathrm{KCl}, 10.8 \mathrm{mM}$ of $\mathrm{CaCl}_{2}, 55.5 \mathrm{mM}$ of $\mathrm{MgCl}_{2}$ and $2 \mathrm{mM}$ of $\mathrm{NaHCO}_{3}$ in deionized water (DIW) and then adjusting the $\mathrm{pH}$ to 8 . The ionic strength of ASW is 694.7 mM.

\section{Methods}

Substrate preparation. A cleaned glass vial was filled with $40 \mathrm{~mL}$ of the dip coating brine and $20 \mathrm{~mL}$ of the $2 \mathrm{mM}$ d-SA solution in decane. After equilibrating for 15 minutes, the vial was placed in a water bath at $60^{\circ} \mathrm{C}$. The substrate was initially immersed below the brine-oil interface and then pulled out at a velocity of $1 \mu \mathrm{m} / \mathrm{sec}$ by clamping it to a motorized long-distance translational stage (Thorlabs). After the dip-coating, the residual $\mathrm{n}$-decane and water on the substrates were blown off with a nitrogen gun. The sample was then dried in a vacuum oven at $40^{\circ} \mathrm{C}$ for 3 hours. 
The d-SA multi-layer is expected to be held together and bound to the substrate via calcium cation bridges ${ }^{[34]}$. Mineral oil was then spin-coated onto the d-SA coated $\mathrm{mica} /$ silica substrate. The spin coating parameters were: $30 \mathrm{~s}$ at $1000 \mathrm{rpm}$ followed by $60 \mathrm{~s}$ at $5000 \mathrm{rpm}$. The oil film formed on the d-SA coated substrate breaks up into small droplets when submerged in ambient brine.

AFM Imaging. Atomic Force Microscopy topography imaging (Bruker Dimension Icon) was carried out in air to characterize the thickness of the deposited d-SA layer. The images were made at a resolution of $512 \times 512$ pixels.

Confocal Raman imaging. Raman measurements were carried out using a WiTec alpha $300 R$ Raman microscope connected to a $532 \mathrm{~nm}$ laser. A $600 \mathrm{~g} / \mathrm{mm}$ grating was used, which provided a spectral resolution of around $2.3 \mathrm{~cm}^{-1}$. A CCD camera $(1600 \times 200$ pixels, $16 \mu \mathrm{m}$ pixel size, Andor Newton) was used for detection of the scattered photons. For high spatial as well as depth resolution, a $63 \mathrm{x}$ water immersion objective (Zeiss W "N-Achroplan”, Numerical Aperture (NA)=0.9, Working Distance $=2.4 \mathrm{~mm}$ ) was chosen. The laser power at the sample was measured using an optical power meter (ThorLabs) and was kept at $18.4 \mathrm{~mW}$ under the objective for all scans. Mapping was performed by raster scanning over a 50 x 50 x $30 \mu \mathrm{m}^{3}$ volume (resolution: $50 \times 50 \times 30$ pixels) for mica and a $100 \times 100 \times 20 \mu \mathrm{m}^{3}$ volume (resolution: $50 \times 50 \times 20$ pixels) for silica. The integration time used was 0.1 (or 0.05 ) $\mathrm{s} /$ pixel, facilitating a $3 \mathrm{D}$ scan in $\sim_{2}$ ( or 1 ) hour $(\mathrm{s})$.

Fluid exchange and $3 \mathrm{D}$ Raman imaging. Initially, the mica/silica $+\mathrm{d}-\mathrm{SA}+$ oil system is immersed in $15 \mathrm{~mL}$ of artificial sea water (ASW) in a petri-dish and allowed to equilibrate with the ambient high salinity brine for an hour. Next, a $3 \mathrm{D}$ Raman scan is performed at an appropriate region. The brown cube in Figure 5.3(a) denotes the collected ${ }_{4} \mathrm{D}$ data, where every smaller cube denotes a single pixel raw Raman spectrum. The ASW is then diluted with DI Water, by three times replacing $12 \mathrm{ml}$ of the ASW with an equal volume of DIW. As a consequence, the ionic strength of the ambient brine reduces from $694.7 \mathrm{mM}$ to $5.5 \mathrm{mM}$. After aging the sample in this low 
salinity brine for one hour to attain equilibrium, ${ }_{3} \mathrm{D}$ Raman imaging is done at the same location.

Pre-processing 3D Raman data. Raster scanned Raman imaging is generally a slow process and a typical Raman spectrum can take several hundreds of milliseconds to several seconds per pixel, depending on the material. To improve the SNR of the acquired hyperspectral Raman dataset, ai-CRM is employed ${ }^{[25]}$. The increase in SNR is achieved via a Principal Component Analysis (PCA) based denoising algorithm. Raman image maps are made by integrating the area under the band of interest for every pixel (or voxel): from 55 to $327 \mathrm{~cm}^{-1}$ for silica, 2000 to $2300 \mathrm{~cm}^{-1}$ for d-SA, 2800 to $3000 \mathrm{~cm}^{-1}$ for oil, 3000 to $3550 \mathrm{~cm}^{-1}$ for water and 3560 to $3700 \mathrm{~cm}^{-1}$ for mica.

\subsection{Results and Discussion}

Clean mica/silica substrates were coated with a 50-100 $\mathrm{nm}$ multi-layer of deuteratedstearic acid (d-SA) by dip-coating through an oil-brine interface as shown schematically in Figure 5.2(a). A detailed study of the hydrophobic stearic acid layers formed under these conditions has been reported elsewhere ${ }^{[37]}$. After cleaning and drying the d-SA coated substrates (Figure 5.2(b)), mineral oil (henceforth referred to as just "oil") was spin-coated onto the d-SA coated substrates as shown schematically in Figure 5.2(c). AFM topographical characterization of the d-SA multi-layer on silica, as shown in Figure 5.2(d), reveals that the layer is heterogenous and a zoom-in of the visible "flat" regions in Figure 5.2(d) (at cross-hair), depicted in Figure 5.2(e), suggests the presence of a monolayer of d-SA $(\sim 2.6 \mathrm{~nm})$ as the underlying layer in this multi-layer deposit.

Figure 5.3(a) shows the schematic of the fluid exchange protocol for in-situ observation of the surfactant desorption-mediated wettability alteration using ai-CRM. Initially, the mineral + d-SA + oil system is immersed into ambient Artificial Sea Water 


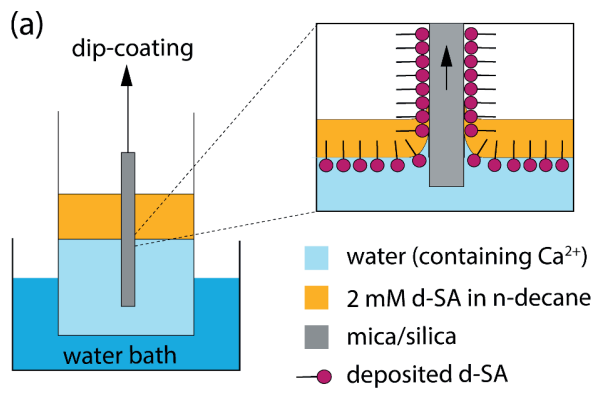

(d)

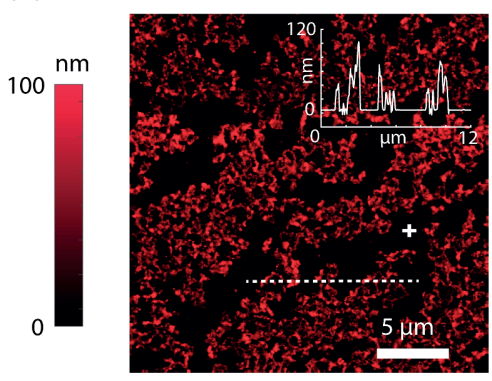

(b)

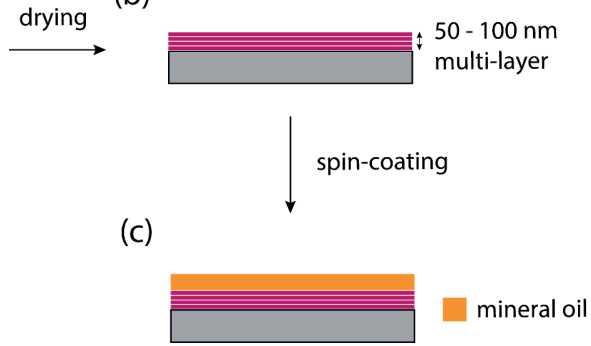

(e)

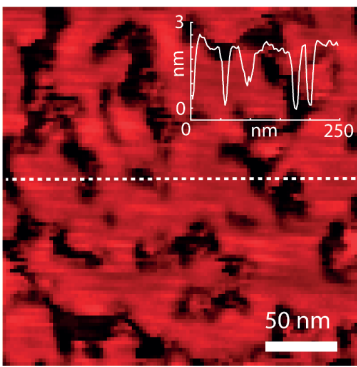

$\mathrm{nm}$

4

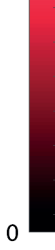

Figure 5.2: Schematic of the (a) dip-coating protocol for the deposition of d-SA on a mica/silica substrate, (b) deposited d-SA multi-layer, 50-10o nm thick after drying, (c) spin-coating of mineral oil on the d-SA coated substrate. (d) AFM height map of the deposited d-SA layer on silica, inset: height profile across the white dashed line. (e) AFM height map of a "flat" region at the cross hair shown in (d), showing the presence of a $\sim 2.6 \mathrm{~nm}$ layer of $d-S A$, inset: height profile across the white dashed line. 
(ASW) in a petri-dish and allowed to equilibrate for one hour. ASW was chosen as the high salinity brine phase in contact with the oil layer. The oil forms a network of interconnected films as shown in the xy optical image in the inset of Figure 5.3(a). After this, the ambient ASW is diluted with DI water, and within a time span of $20-$ 40 minutes, the film dewets into droplets as shown in the right inset of Figure 5.3 (a). After aging the sample in this low salinity brine for one hour to attain equilibrium of the contact angle, another ${ }_{3} \mathrm{D}$ Raman scan is performed at the same location, to monitor the response of the system to a lowered salinity of the ambient brine. Figure $5 \cdot 3$ (b) shows an example of a single pixel raw spectrum of the system being investigated and Figure 5.3 (c) shows the same spectrum denoised using ai-CRM. Details of the technique and its implementation for ${ }_{3} \mathrm{D}$ Raman data can be found in chapter 2 of this thesis.

Below we demonstrate the capability of ai-CRM for the simultaneous measurement of material redistributions and the corresponding contact angle changes. By applying this to a model system for low salinity water flooding EOR in sandstone reservoirs, we also gain new insights into the microscopic mechanism underlying the wettability alteration. The quartz and clay fractions of sandstone reservoirs are represented by silica and mica, respectively.

\subsubsection{Reactive wettability alteration on mica}

We first consider the wettability alteration on mica. 3 D Raman images of mica, oil, water and d-SA were measured for a typical oil droplet, by making xy scans at different $\mathrm{z}$ depths and selecting their respective Raman bands. As illustrated in the denoised spectrum in Figure 5.3(c), even a single pixel Raman spectrum is rich in information and shows good discernibility of the components, facilitating simultaneous imaging.

Confocal volumetric scanning using Raman microscopy has the advantage of mapping the local microscopic oil droplet shape as well as the distribution of compon- 
(a)

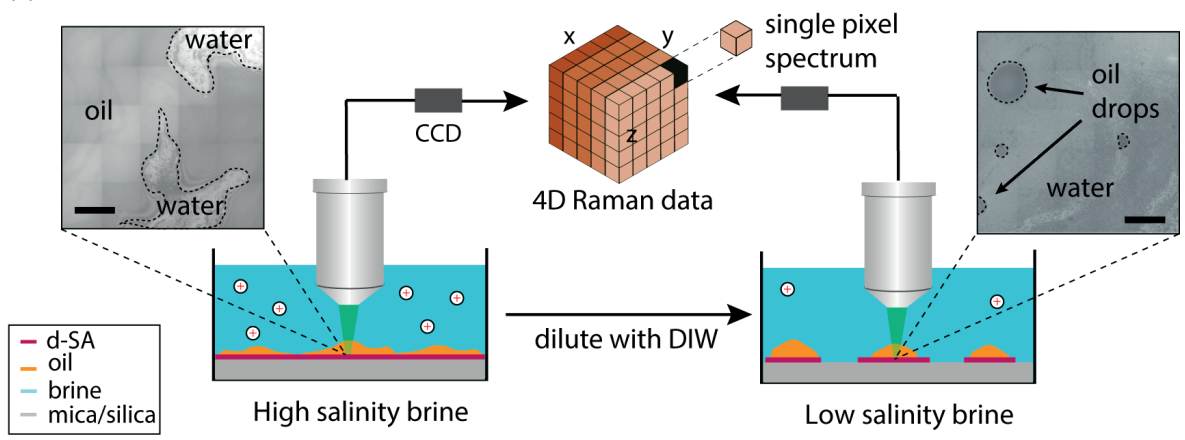

(b)
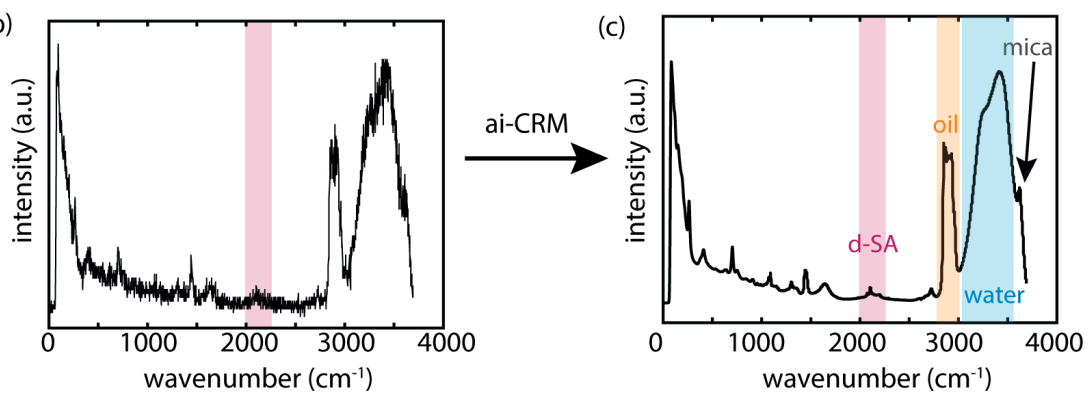

Figure 5.3: (a) Fluid exchange protocol for studying the surfactant mediated reactive wettability alteration using ai-CRM. The optical images show the distribution of oil on the substrate in the respective ambient brine condition. Scale bar corresponds to $0.2 \mathrm{~mm}$. (b) An example of a single pixel raw Raman data and (c) same data as (b), but after ai-CRM denoising. 

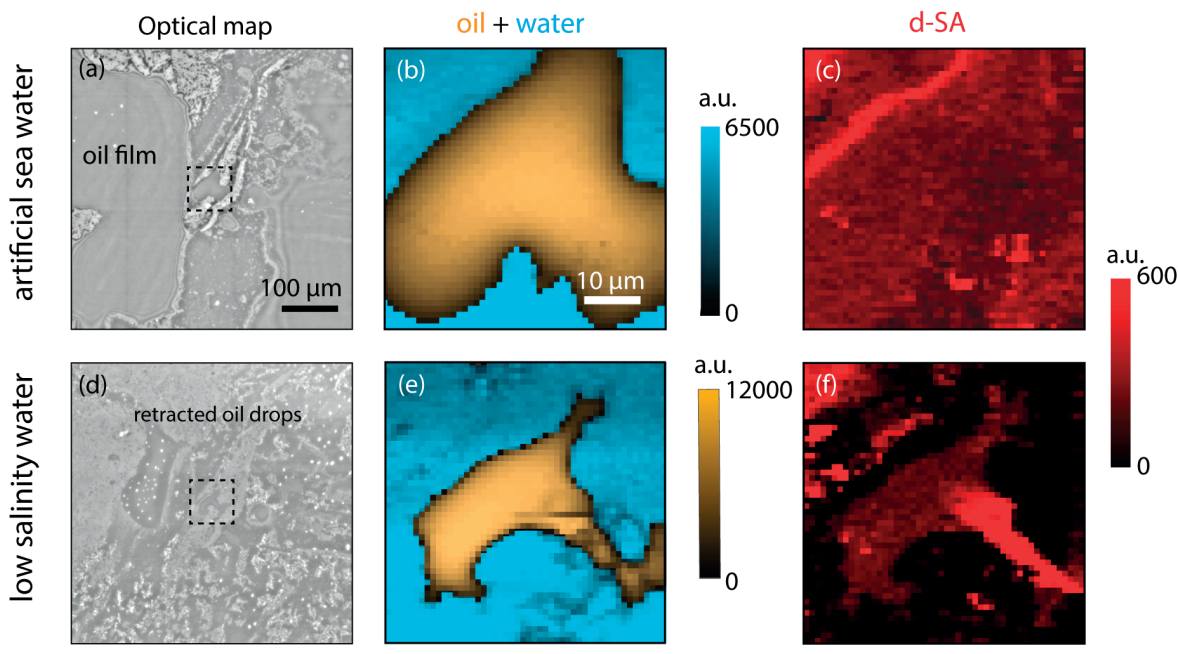

Figure 5.4: Optical and Raman xy maps of oil (yellow), water (blue) and d-SA (red) on a mica substrate. The top row (panels (a), (b), (c)) corresponds to an ambient of $A S W$, while in the bottom row (panels $(d),(e),(f))$ the brine salinity has been reduced. The optical maps ((a) vs. (d)) shows large area oil films retracting into oil droplets when the salinity is reduced. The dashed box denotes the region where Raman imaging was performed. The before-after comparisons of the Raman maps ((b) vs. (e), (c) vs. $(f))$ clearly reveal that the oil drop retracts and rearranges the initially conformally covered d-SA layer into its footprint. 
ents in 3 dimensions, which is not possible with standard optical contact angle goniometry. Orthogonal sectioning parallel to the mica plane, at a depth corresponding to the d-SA-oil interface, can be used to visualize the xy component distributions. Figure 5.4 shows the reflection mode xy optical and Raman maps of oil, water and d-SA. The large area optical map in Figure 5.4(a) depicts the presence of thin oil patches in (high salinity) ASW. On reducing the brine salinity (Figure 5.4(d)), these extended oil patches retract and break up into droplets. Figure 5.4(b) shows a representative irregularly shaped oil drop (corresponding to the dashed box in Figure 5.4(a)) in its initial state in ambient ASW. The corresponding Raman map of d-SA shows that the entire solid surface is covered by surfactant (Figure 5.4(c)). Yet, the layer displays substantial heterogeneity, which probably contributes to the irregular shape of the oil drop.

Figure 5.4(e) and (f) depict the distribution of oil-water and d-SA in response to lowered ambient brine salinity. Over a period of 20-40 minutes after the fluid exchange, the oil droplet retracts and attains a new equilibrium configuration. As seen in Figure 5.4(f), the d-SA layer also reorganizes, conforming to the new footprint of the retracted droplet, as evident from the co-localization of the oil droplet and the d-SA distributions. The xy Raman maps clearly suggest a reactive wettability alteration, facilitated by the detachment of the d-SA layer in response to lowered ambient brine salinity. We must mention that we cannot resolve what happens dynamically between the two cases, but it is plausible that the retraction of the oil droplet starts with the release of the d-SA layer, as corroborated by the co-localization of the two distributions.

Orthogonal sectioning of the ${ }_{3} \mathrm{D}$ volumetric Raman data perpendicular to the mica interface enables the visualization of the local contact angle. The $\mathrm{xz}$ cross-section Raman maps of the same system, depicted in Figure 5.5, provide additional insight into the mechanism of wettability alteration. The xz map was taken at an arbitrary line passing through the center of the xy maps shown in Figure 5.4. The cross-section 

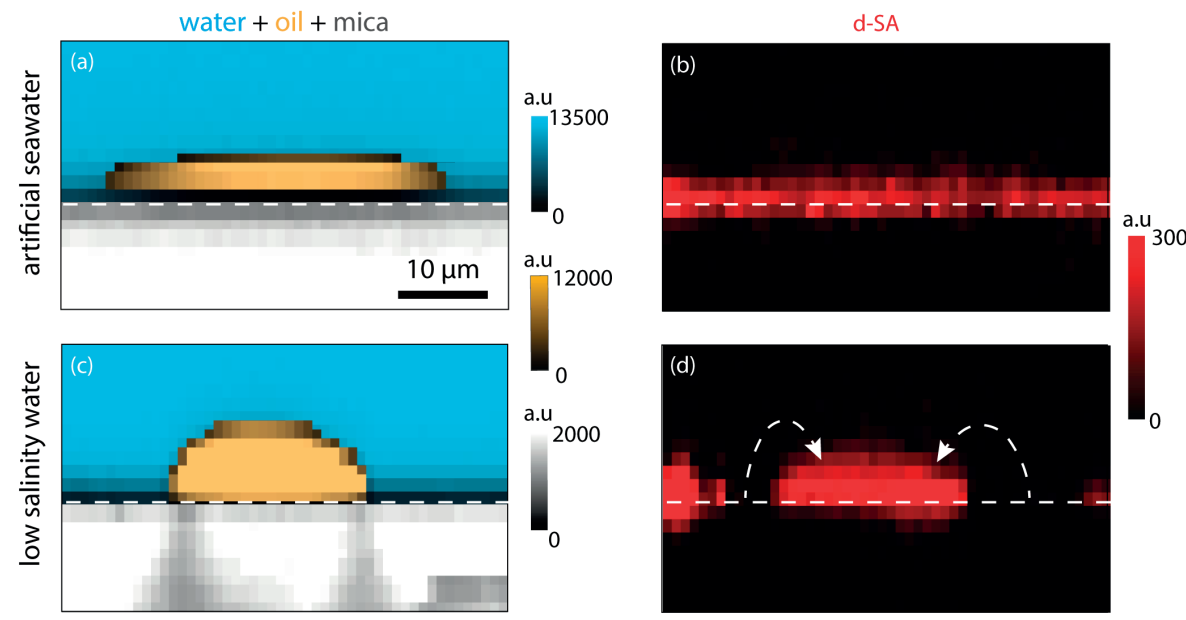

Figure 5.5: Raman xz maps of oil (yellow), water (blue), mica (grey) and d-SA (red). Ambient brine is initially ASW ((a) and (b)), while it has been diluted with DIW in ( (c) and (d)). Raman imaging in $3 D$ helps in visualizing contact angle changes and $d$-SA layer detachment and dissolution (shown by the dashed arrows) into the oil phase. 
maps clearly show the configuration of the system studied. The mica is coated with a layer of d-SA, atop of which an oil droplet sits in ambient brine. Figure 5.5 (a) and (b) show the distribution of the components with ASW as the ambient, whereas Figure 5.5 (c) and (d) show the distribution after the ambient salinity reduction. Two key observations can be made: (1) The droplet retracts its TPCL, thereby increasing its contact angle and ( 2 ) the d-SA layer is 'swept-up' from the substrate by the retracting TPCL and appears to be now dissolved in the retracted oil droplet after the salinity is reduced. This 'sweep-up' mechanism is further illustrated via optical microscopy in Figure 5.6. Qualitatively, we have repeatedly observed that thicker surfactant layers tend to act as strong pinning sites for the contact line, such that droplet retraction is not observed when the salinity is lowered. Thinner layers of d-SA induce less pinning and display a stronger low salinity response. Figure 5.6(a) shows thicker, heterogeneous underlying d-SA layers, and the droplet does not recede after the salinity is lowered. Figure 5.6(b) shows a thinner region on the same substrate. By observing the trail of bare mica behind the droplet, it is evident that these regions showed the low salinity effect clearly.

Previously, ai-CRM has been used to monitor the release of oil droplets from clay due to a flow of lowered salinity brine in a microfluidic channel ${ }^{[38]}$, however that study had to speculate about the role of the surfactant in the wettability alteration. In this work we show, for the first time, the nexus between the reorganization of the surfactant and wettability alteration. It not only underlines the earlier given mechanistic explanation of wettability alteration via surfactant desorption, but also reveals that the presence of an oil phase provides a pathway for the d-SA adsorbed on the "clay" to reorganize. To investigate the effect of lowered brine salinity on the dissolution of d-SA layer without an oil phase, we carried out in-situ AFM topography imaging in a liquid cell with ambient brine. The d-SA layer was aged in ASW for three hours and then imaged as shown in Figure 5.7(a). Next, the salinity was reduced by exchanging the ASW with DIW as mentioned in the experimental section and another image was 

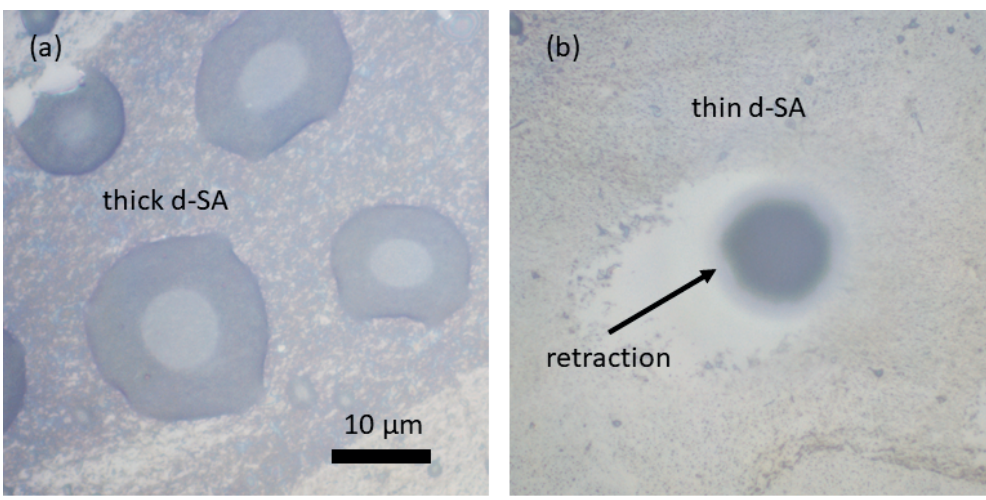

Figure 5.6: Optical micrographs of oil droplets on mica substrates after the salinity is lowered. (a) Thicker regions tend to pin the droplet severely, and no change in contact angle is observed. (b) Thinner regions, identified by their lower optical contrast, clearly show the low salinity response.

taken at the same region, after 12 hours, as shown in Figure 5.7(b). Certain regions (denoted by the dashed circles in Figure 5.7) showed signs of d-SA dissolution due to lowered ambient brine salinity. This is consistent with the mechanism proposed, and hints that the presence of an oil phase is crucial for the d-SA layer to dissolve and reorganise faster, thus accelerating the process of reactive wetting.

\subsubsection{Reactive wettability alteration on silica}

Silica samples qualitatively display the same behaviour as the mica substrates discussed so far. In the initial state, the oil is less widely spread across the substrates and forms drops rather than extended patches in ambient ASW, as seen in Figure 5.8(a) and (b). Figure 5.8(c) shows that the d-SA layer is heterogeneous, but can be detected essentially everywhere on the substrate. Upon reducing the salinity, the macroscopic optical images clearly show a retraction of the oil drops, in agreement with the Raman maps of the oil distribution (Figure 5.8(d) and (e), respectively). Like in the case of mica, preferential removal of d-SA is observed in regions that are not 
(a) artificial sea water

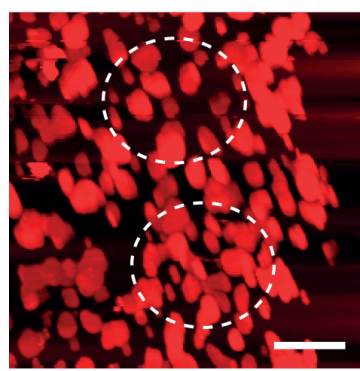

(b) low salinity water

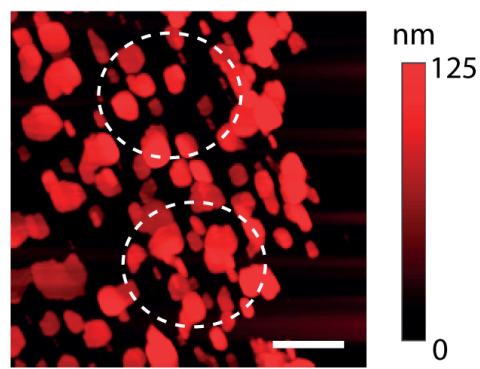

Figure 5.7: AFM topography characterization of d-SA layer in (a) ASW and (b) low salinity water after 12 hours of aging. The dashed circle shows regions where the d-SA layer has desorbed into the ambient brine on lowering the salinity. Scale bars: $2 \mu \mathrm{m}$.

covered by oil drops in the final state, shown in Figure 5.8(f). This is further corroborated by the xz cross sections shown in Figure 5.9, where the coupling between (1) the change in substrate wettability and (2) the removal and dissolution of the d-SA into the oil phase by the retracting contact line are visible. This also means that our mechanistic explanation for low salinity induced wettability alteration on mica applies to silica alike. However, unlike mica, we have repeatedly observed incomplete co-localization on silica, suggesting a stronger adhesion of the surfactant molecules to silica which leads to an incomplete removal of d-SA by the retracting oil droplet. The observation that the removal of the layer is more pronounced and homogeneous on mica than on silica is consistent with our earlier observations on wettability alteration as a function of brine composition ${ }^{[35]}$. Also the different heterogeneities of the d-SA layers deposited on mica and silica may have played a role. To probe this difference, the d-SA layers were characterized using an AFM post-coating. This is shown in Figure 5.10. d-SA layers on mica are less rough and heterogeneous (Figure $5.10(\mathrm{c})$ ) as compared to the layers on silica (Figure 5.10(a)). A zoom-in of these regions, depicted in Figure 5.10(b) and (d) also shows the difference in coverage of the thinner d-SA layers. This can contribute to different hysteresis on these substrates, 

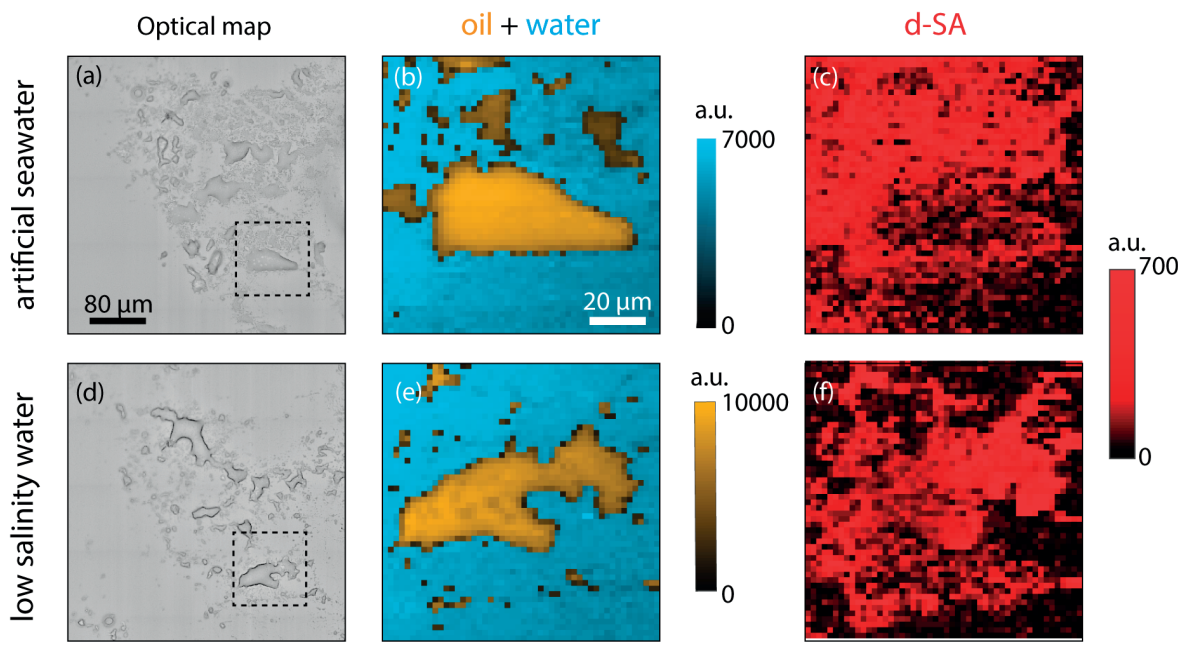

Figure 5.8: Optical and Raman xy maps of oil (yellow), water (blue) and d-SA (red) on a silica substrate. The top row (panels (a), (b), (c)) corresponds to an ambient of ASW, while in the bottom row (panels $(d),(e),(f))$ the brine salinity has been reduced. The dashed box in the optical maps denotes the region where the Raman maps were performed. The before-after comparisons of the Raman maps ((b) vs. (e), (c) vs. $(f))$ clearly reveal that the oil drop retracts and reorganizes the d-SA layer. This behaviour is similar to the reactive wettability alteration observed on mica, however less pronounced.

which would be an explanation of why the contact angle change is also less on silica as compared to mica.

\subsubsection{Surfactant redistribution post wettability alteration}

After the salinity of the ambient brine was lowered, the oil droplets were aged for an extra hour after the contact angle had stopped changing, see Figure 5.11. While the drop shape remained constant - presumably because the contact line was pinned to surface heterogeneities - surfactant redistribution still continued. The partial decomposition of the d-SA layer at the substrate-brine interface remained more pronounced for mica than for silica surfaces. Yet, after the extended waiting time both 

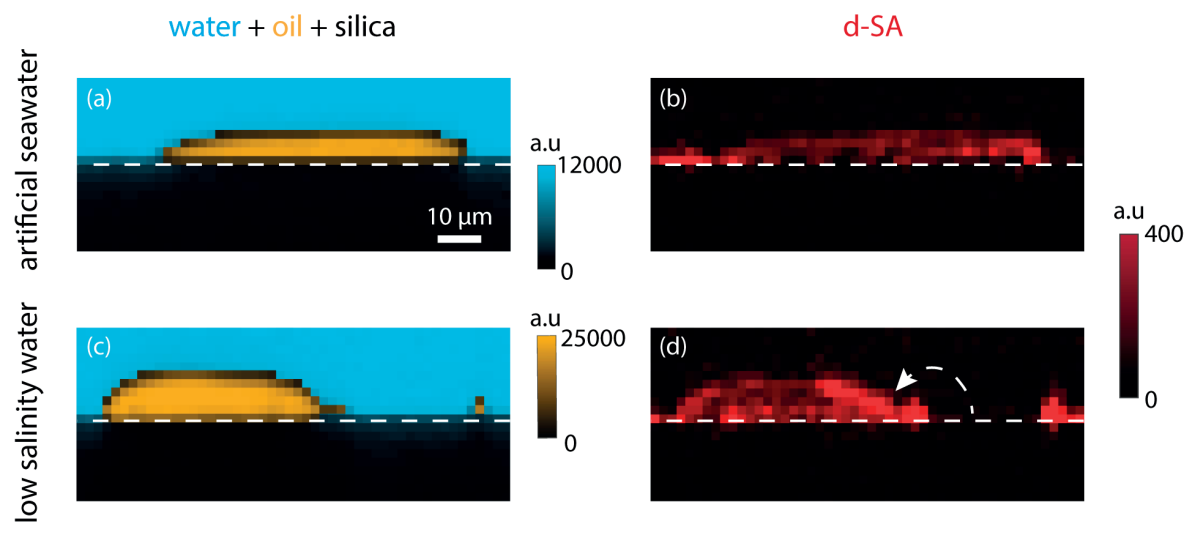

Figure 5.9: Raman xz maps of oil (yellow), water (blue) and d-SA (red) on silica: (a),(b) in ASW and (c), (d) in response to lowered ambient brine salinity. Similar to the behavior on mica, the TPCL retracts and the d-SA layer detaches and dissolves into the oil phase.

systems displayed an accumulation of d-SA along the oil-brine interface, as shown in the CRM images Figure 5.11(b) and (e) as well as the spectra at the various interfaces indicated in Figure 5.11 (c) and (f). These layers were absent in high salinity ambient brines (see insets of Figure 5.11(b) and (e)). Based on the relatively weak sensitivity of Raman scattering, we conclude that this layer at the $\mathrm{O} / \mathrm{W}$ interface has a thickness of at least a few tens of $\mathrm{nm}$. This layer is reminiscent of the interfacial precipitation of Ca-stearate between solutions of stearic acid in decane and $\mathrm{Ca}$-containing aqueous brines reported previously ${ }^{[39]}$. This material formed a stable bulk phase leading to up to micrometer thick solid skins at $\mathrm{O} / \mathrm{W}$ interfaces. Assuming a similar thermodynamic stability for the interfacial layers observed in the present experiments, it is not surprising that the formation of these layers as well as the concomitant contact angle change are irreversible. In order to probe the effect of salinity reversal on the wettability alteration, $x z$ Raman mapping was performed after a sequence of salinity switches on mica. The resulting contact angle change and surfactant reorganization is shown in Figure 5.12. As seen, neither the $\mathrm{O} / \mathrm{W}$ nor the $M / W$ interface shows significant changes in d-SA occupancy after salinity reversal (Figure 5.12 (d) vs. (f)). The 

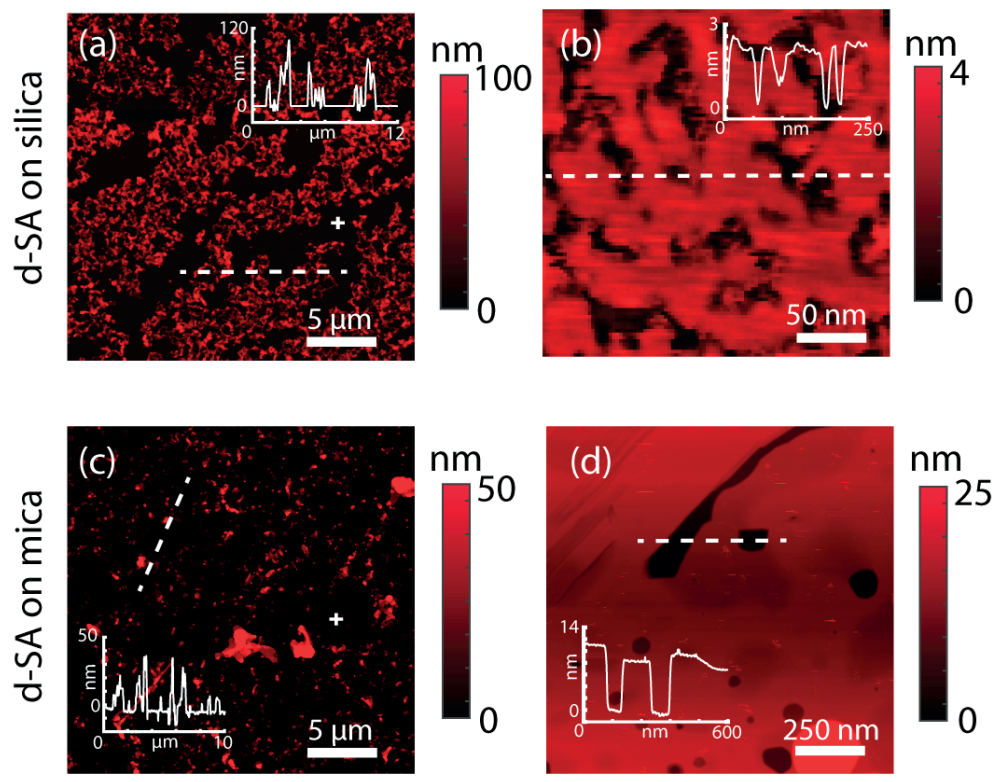

Figure 5.10: AFM topography maps of d-SA deposited on (a) silica and (c) mica. (b) and (d) show a zoom-in at the regions depicted by the cross-hair in (a) and (c), respectively. Insets show the height profile across the white dashed line. 

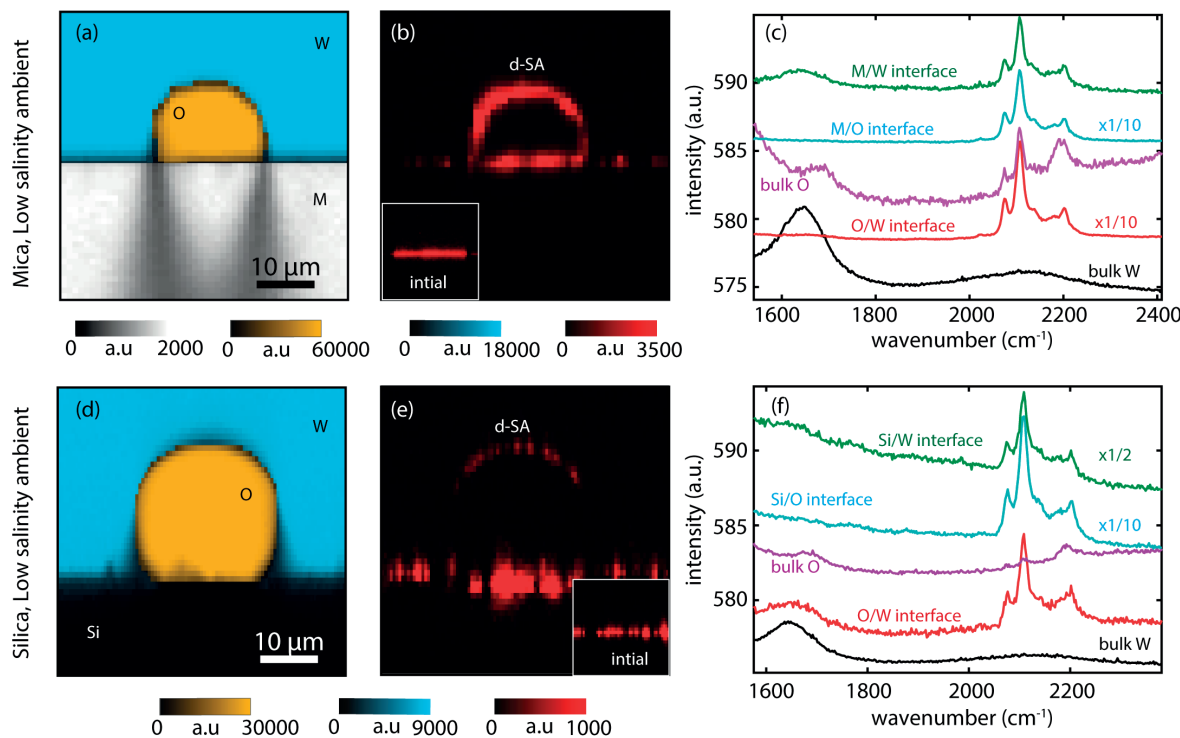

Figure 5.11: $x z$ Raman maps of the component distributions, one hour after the contact angle equilibration, on (a), (b) mica and (d), (e) silica, respectively. The insets in panel (b) and (e) show the initial d-SA configuration under ASW brine. The mean spectrum at each interface and bulk phase, depicted in (c) for mica and in $(f)$ for silica indicate the presence of $d-S A$ as a skin at the oil-water interface.

initial state with the thick spin-coated d-SA layer was a kinetically arrested (i.e. nonequilibrium) configuration. Once decomposed by exposure to low salinity brine, there is no driving force to restore the original configuration. The thick (presumably $\mathrm{Ca}^{2+}$-stabilized) layers of $\mathrm{d}$-SA at the $\mathrm{O} / \mathrm{W}$ interface are sufficiently stable and do not spontaneously spread onto the solid surface again. This conclusion is consistent with earlier measurements on interfacial precipitation of Ca-stearate layers at brine-oil interfaces ${ }^{[39]}$.

Notably, compared to silica the O/W interfacial accumulation of d-SA is more pronounced for mica, where also the d-SA removal from the substrate is more efficient. These observations are consistent with the idea that the removed d-SA from the solid- 


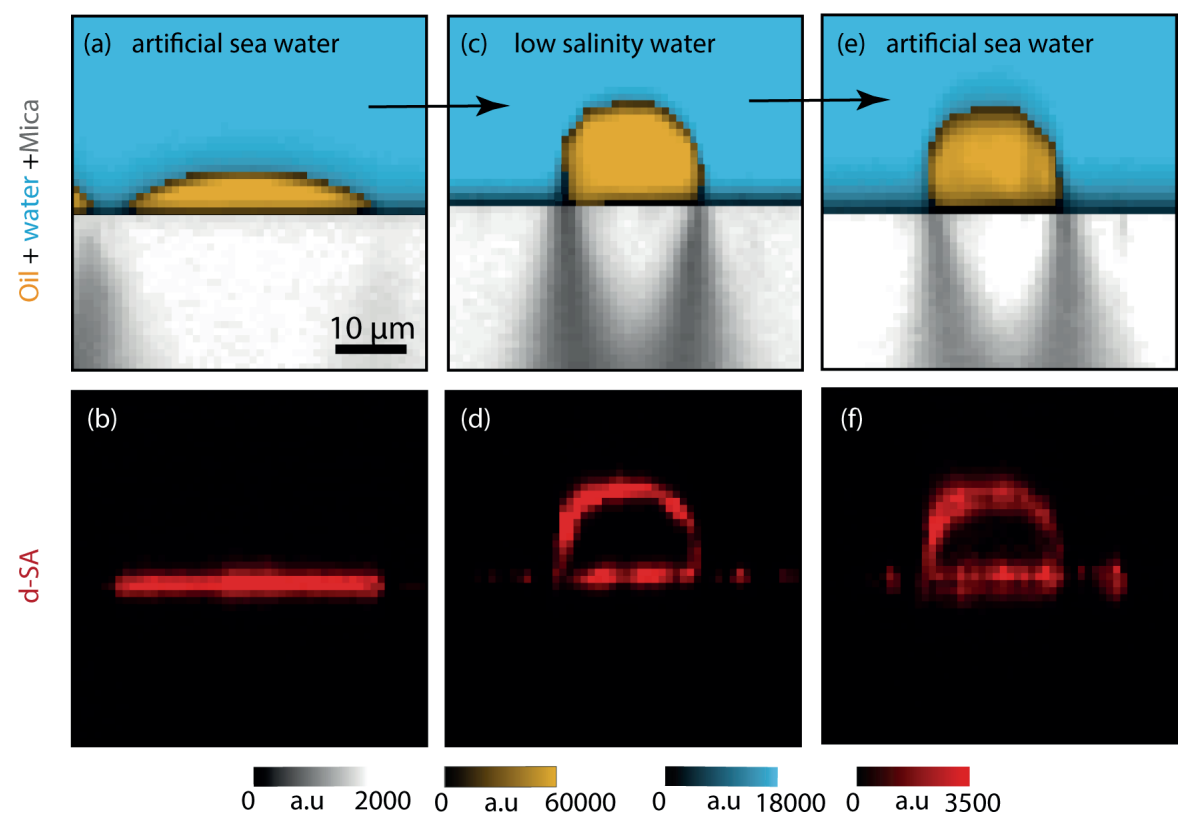

Figure 5.12: $x z$ cross-section Raman maps of (top row) oil, water and mica, and (bottom row) d-SA after two ambient brine salinity switches. (a), (b) Initially, from the highsalinity case (ASW), (c), (d) the salinity is lowered and after performing the Raman imaging, the salinity is switched back to $(e),(f)$ high salinity again. 
liquid interface is the source of the material accumulating at the $\mathrm{O} / \mathrm{W}$ interface. However, the mechanistic path of the transfer remains unclear, the originally deposited layer might either become transferred directly from the solid substrate to the $\mathrm{O} / \mathrm{W}$ interface or they might first dissolve in the oil drop and subsequently re-precipitates at the $\mathrm{O} / \mathrm{W}$ interface as previously shown ${ }^{[39]}$. Figure 5.11 (c) and (f) show a small d-SA signal arising from the bulk of the oil, but none from the water phase. The latter is not surprising, as the solubility of d-SA in water should at least be an order of magnitude lower as compared to oil at the same temperature ${ }^{[40][41]}$.

\subsubsection{Substrate-dependent contact angle change}

The xz cross sections of the Raman maps shown in Figure 5.5 and Figure 5.9 already demonstrated the qualitative variation of the wettability with salinity. To obtain a quantitative estimate the of contact angle, we fitted a circle through the droplet surface obtained from the Raman cross section using Image (version J2, Fiji) as shown in Figure 5.13 (a) (obviously, this procedure yields both the contact angle in the oil phase, $\theta_{\text {oil }}$ and its complement, the water contact angle $\left.\theta_{\mathrm{W}}=180^{\circ}-\theta_{\text {oil }}\right)$. To account for both the known uncertainties in the extraction of surface profiles from the Raman data ${ }^{[42]}$ and for variations in the contact angle caused by the heterogeneity of the substrate, we repeated this procedure for 6 different locations along the TPCL and averaged the resulting contact angles. Notwithstanding the uncertainties of the procedure, it is obvious from the results shown in Figure 5.13(b) that a significant reduction of the water contact angle is found for both substrates upon reducing the ambient salinity. Consistent with the qualitative description given above, the contact angle reduction for mica $\left(\sim 55^{\circ}\right)$ is substantially more pronounced than for silica $\left(\sim 25^{\circ}\right)$. 
(a)

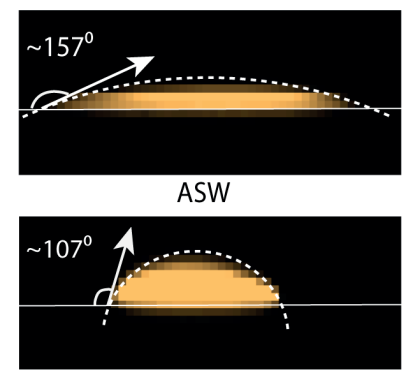

low salinity water (LSW) (b)

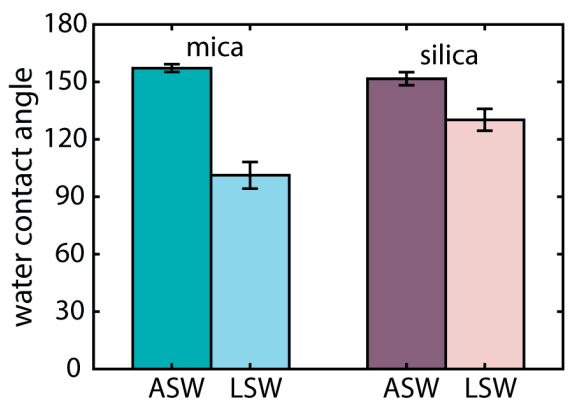

Figure 5.13: (a) Circular fitting of droplet shape and extraction of the water contact angle using Raman xz cross-section maps. (b) Changes in water contact angle observed on mica and silica substrates in response to lowered ambient brine salinity.

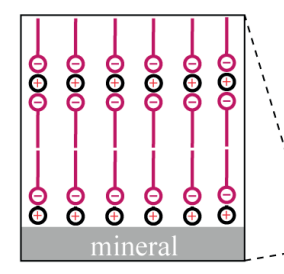

$\multimap d-\mathrm{SA}^{-} \odot \mathrm{Ca}^{2+}$

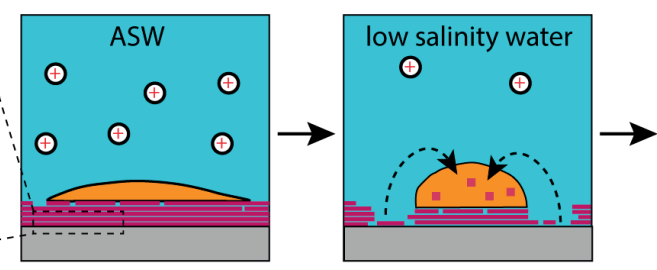

(a) stable d-SA (b) wettability change, d-SA dissolution into oil

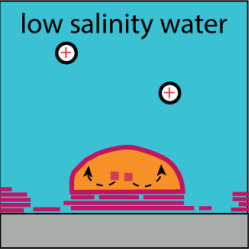

(c) skin formation, irreversible

Figure 5.14: Suggested mechanism for the reactive wettability alteration: (a) Configuration of the oil droplet atop the stable d-SA multi-layer in ASW. The zoom-in represents the widely believed cation bridging mechanism of polar organics to the mineral substrate. (b) Dissolution of the d-SA into the oil phase by the retracting TPCL, causing a change in wettability of the mineral in low salinity water. (c) d-SA skin formation at the $\mathrm{O} / \mathrm{W}$ interface post contact angle stabilization. This skin formation is irreversible on switching back to high salinity (refer Figure 5.12). 


\subsubsection{Wettability alteration mechanism}

The experiments discussed above illustrate the unique capability of ai-CRM for gaining microscopic insights into reactive wettability alteration. Drawing cues from the observed contact angle changes and reorganization of the d-SA layer (in response to lowered ambient brine salinity), we propose a mechanism for this reactive wetting, shown schematically in Figure 5.14. Briefly, the initial spin-coated oil film attains an equilibrium in ASW, by breaking up into droplets which sit atop the d-SA multilayer. The relatively low contact angle of the oil droplets originates from the hydrophobic tails of d-SA, which are collectively sticking out in the same direction. This self-assembly of the d-SA molecules is facilitated by (probably divalent ${ }^{[43]}$ ) cation bridges between the carboxylic acid groups and the mineral substrate. The assembled hydrophobic monolayer either provides a hydrophobic surface directly, or serves as a template for the deposition of additional bilayers, which also have their hydrophobic tails sticking out (see zoom-in, Figure 5.14(a)). On lowering the ambient brine salinity, part of the bridging cations get released into the brine, thereby triggering a partial desorption of d-SA layer. This desorption is assisted by the availability of an oil phase, in which the d-SA molecules can dissolve. We cannot claim that no d-SA layer is left behind on the substrate in the regions of droplet retraction, as the remaining layer could be too thin to be detected within the resolution of the confocal Raman microscope. The ambient brine then seeps into the hydrophilized region, altering the contact angle of the oil droplet as shown schematically in Figure 5.14 (b). Thus, the substrate changes from primarily oil-wet to partially water-wet. Post stabilization of the contact angle, the oil acts as a reservoir and transfer medium for d-SA molecules, allowing the latter to accumulate at the oil-water interface and leading to the formation of a skin which cloaks the oil droplet. This is shown schematically in Figure 5.14(c). 


\subsection{Conclusion}

In this work, we demonstrated the utilization of ai-CRM for characterizing reactive wettability alteration mechanisms by studying a specific example of the phenomenon occurring in low salinity water flooding EOR. Compared to the conventional techniques of probing the wetting of droplets on substrates ${ }^{[14-20]}$, we show that the benefits of using ai-CRM are twofold: (a) it can be used to spectroscopically monitor changes in multi-component distribution and reorganization triggered by an external stimulus and (b) it can also be used to perform label-free micron-sized droplet visualization enabled by ${ }_{3} \mathrm{D}$ Raman mapping. However, the average time taken per $3 \mathrm{D}$ Raman scan using ai-CRM is approximately 1 hour. In our case this was insufficient to follow the ${ }_{3} \mathrm{D}$ evolution of the droplet (after reducing ambient salinity), which takes 20-40 minutes. To actually follow the evolution of the drop shape, one could reduce the scanning to two-dimensional cross sections in the $\mathrm{xz}$ or $\mathrm{yz}$ directions. While this would speed up data acquisition by approximately 50 times, it would sacrifice the full $3 \mathrm{D}$ information, which is not desirable for non-symmetric drops in the present experiments. Specific to the model system studied, we show experimentally for the first time a nexus between reorganization of a surfactant layer and the consequent wettability alteration of the substrate due to change in ambient brine concentration. We also propose a mechanism for understanding the phenomenon in the same system. Although CRM is often considered notorious for being a slow technique, this work shows that by probing the right length and time-scales, ai-CRM can be a powerful technique to image and understand similar systems holistically.

Because of the broad applicability of Raman imaging in general, our analysis method is well-suited to mechanistically examine other wettability alterations, like the recent study on the adaptive wetting of polydimethylsiloxane ${ }^{[44]}$. A variety of smart materials are designed to be responsive to an external stimulus, and thereby change the wettability of the substrate ${ }^{[2]}$. A special class of these smart materials is that of polymer brushes, which can change configuration, depending on the $\mathrm{pH}$ or ionic 
strength $^{[45,46]}$. With ai-CRM, it should be possible to characterize this configurational change by monitoring the low wavenumber $\left(<200 \mathrm{~cm}^{-1}\right)$ Raman bands, which originate from skeletal deformation modes or inter-chain interactions (refer Chapter $\left.9^{[47]}\right)$. Besides that, ai-CRM can also be used for simultaneous microcontact angle goniometry and spectroscopy in other fields of interfacial science, like two-phase wetting in (Raman transparent) porous media, wetting on composite substrates (e.g. polymer blends) and surface wettability of micron-sized particles and fibers.

\section{Bibliography}

[1] H.-J. Butt, R. Berger, W. Steffen, D. Vollmer, and S. A. Weber, "Adaptive wetting —adaptation in wetting," Langmuir, vol. 34, no. 38, pp. 11292-11304, 2018.

[2] F. Guo and Z. Guo, "Inspired smart materials with external stimuli responsive wettability: a review," RSC advances, vol. 6, no. 43, pp. 36623-36641, 2016.

[3] A. Hozumi, L. Jiang, H. Lee, and M. Shimomura, Stimuli-Responsive Dewetting/Wetting Smart Surfaces and Interfaces, vol. 11. Springer, 2018.

[4] R. Wang, K. Hashimoto, A. Fujishima, M. Chikuni, E. Kojima, A. Kitamura, M. Shimohigoshi, and T. Watanabe, "Light-induced amphiphilic surfaces," Nature, vol. 388, no. 6641, pp. 431-432, 1997.

[5] J. Gao, X. Yao, Y. Zhao, and L. Jiang, "Lyophilic non-wettable surface based on an oil/water/air/solid four-phase system," Small, vol. 9, no. 15, pp. 2515-2519, 2013.

[6] J. Jiang, J. Gao, H. Zhang, W. He, J. Zhang, D. Daniel, and X. Yao, "Directional pumping of water and oil microdroplets on slippery surface," Proceedings of the National Academy of Sciences, vol. 116, no. 7, pp. 2482-2487, 2019. 
[7] J. Li, N. S. Ha, and R. M. van Dam, "Ionic-surfactant-mediated electrodewetting for digital microfluidics," Nature, vol. 572, no. 7770, pp. 507-510, 2019.

[8] A. K. Kota, G. Kwon, W. Choi, J. M. Mabry, and A. Tuteja, "Hygro-responsive membranes for effective oil-water separation," Nature communications, vol. 3, no. 1, pp. 1-8, 2012.

[9] Z. Dang, L. Liu, Y. Li, Y. Xiang, and G. Guo, "In situ and ex situ pH-responsive coatings with switchable wettability for controllable oil/water separation," ACS applied materials and interfaces, vol. 8, no. 45, pp. 31281-31288, 2016.

[10] M. Xiao, A. Xu, T. Zhang, and L. Hong, “Tailoring the wettability of colloidal particles for pickering emulsions via surface modification and roughness," Frontiers in chemistry, vol. 6, p. 225, 2018.

[1 1 ] P. D. Hallett, "A brief overview of the causes, impacts and amelioration of soil water repellency-a review," Soil and Water Research, vol. 3, no. 1, pp. 521-528, 2008.

[12] E. Graber, S. Tagger, and R. Wallach, "Role of divalent fatty acid salts in soil water repellency," Soil Science Society of America Journal, vol. 73, no. 2, pp. 541$549,2009$.

[13] J. Sheng, "Critical review of low-salinity waterflooding," Journal of Petroleum Science and Engineering, vol. 120, pp. 216-224, 2014.

[14] F. Mugele, T. Becker, R. Nikopoulos, M. Kohonen, and S. Herminghaus, "Capillarity at the nanoscale: an afm view," Journal of adhesion science and techno$\log y$, vol. 16, no. 7, pp. 951-964, 2002.

[15] K. Stöckelhuber, B. Radoev, and H. Schulze, "Some new observations on line 
tension of microscopic droplets," Colloids and Surfaces A: Physicochemical and Engineering Aspects, vol. 156, no. 1-3, pp. 323-333, 1999.

[16] M. Andrew, B. Bijeljic, and M. J. Blunt, "Pore-scale contact angle measurements at reservoir conditions using x-ray microtomography," Advances in Water Resources, vol. 68, pp. 24-31, 2014.

[17] D. I. Yu, H. J. Kwak, H. Noh, H. S. Park, K. Fezzaa, and M. H. Kim, "Synchrotron $\mathrm{x}$-ray imaging visualization study of capillary-induced flow and critical heat flux on surfaces with engineered micropillars," Science advances, vol. 4, no. 2, p. e1701571, 2018.

[18] C. Luo, H. Zheng, L. Wang, H. Fang, J. Hu, C. Fan, Y. Cao, and J. Wang, "Direct three-dimensional imaging of the buried interfaces between water and superhydrophobic surfaces," Angewandte Chemie International Edition, vol. 49, no. 48, pp. 9145-9148, 2010.

[19] H. Rathgen and F. Mugele, "Microscopic shape and contact angle measurement at a superhydrophobic surface," Faraday discussions, vol. 146, pp. 49-56, 2010 .

[20] P. Papadopoulos, L. Mammen, X. Deng, D. Vollmer, and H.-J. Butt, "How superhydrophobicity breaks down," Proceedings of the National Academy of Sciences, vol. 110 , no. 9, pp. 3254-3258, 2013.

[21] R. Craster and O. Matar, "On autophobing in surfactant-driven thin films," Langmuir, vol. 23, no. 5, pp. 2588-2601, 2007.

[22] A. Mazurek, S. J. Pogorzelski, and K. Boniewicz-Szmyt, "Adsorption of natural surfactants present in sea waters at surfaces of minerals: contact angle measurements," Oceanologia, vol. 51, no. 3, pp. 377-403, 2009. 
[23] R. Sharma, R. Kalita, E. R. Swanson, T. E. Corcoran, S. Garoff, T. M. Przybycien, and R. D. Tilton, "Autophobing on liquid subphases driven by the interfacial transport of amphiphilic molecules," Langmuir, vol. 28, no. 43, pp. $15212-$ $15221,2012$.

[24] B. Bera, M. H. Duits, M. C. Stuart, D. Van Den Ende, and F. Mugele, "Surfactant induced autophobing," Soft matter, vol. 12, no. 20, pp. 4562-4571, 2016.

[25] S. Nair, J. Gao, Q. Yao, M. H. Duits, C. Otto, and F. Mugele, "Algorithmimproved high speed and non-invasive confocal Raman imaging of twodimensional materials," National Science Review, 2019.

[26] M. Jackson, J. Vinogradov, G. Hamon, and M. Chamerois, "Evidence, mechanisms and improved understanding of controlled salinity waterflooding part 1: Sandstones," Fuel, vol. 185, pp. 772-793, 2016.

[27] P. C. Myint and A. Firoozabadi, “Thin liquid films in improved oil recovery from low-salinity brine," Current Opinion in Colloid and Interface Science, vol. 20, no. 2, pp. 105-114, 2015.

[28] E. W. Al-Shalabi and K. Sepehrnoori, "A comprehensive review of low salinity/engineered water injections and their applications in sandstone and carbonate rocks," Journal of Petroleum Science and Engineering, vol. 139, pp. 137$161,2016$.

[29] S. Strand, T. Puntervold, and T. Austad, "Water based EOR from clastic oil reservoirs by wettability alteration: A review of chemical aspects," Journal of Petroleum Science and Engineering, vol. 146, pp. 1079-1091, 2016.

[30] P. McGuire, J. Chatham, F. Paskvan, D. Sommer, and F. Carini, "Low salinity oil recovery: An exciting new EOR opportunity for Alaska's north slope," in SPE western regional meeting, Society of Petroleum Engineers, 2005. 
[31] G.-Q. Tang and N. R. Morrow, "Influence of brine composition and fines migration on crude oil/brine/rock interactions and oil recovery," Journal of Petroleum Science and Engineering, vol. 24, no. 2-4, pp. 99-111, 1999.

[32] W. Song and A. R. Kovscek, "Functionalization of micromodels with kaolinite for investigation of low salinity oil-recovery processes," Lab on a Chip, vol. 15, no. 16 , pp. $3314-3325,2015$.

[33] D. J. Ligthelm, J. Gronsveld, J. Hofman, N. Brussee, F. Marcelis, and H. van der Linde, "Novel waterflooding strategy by manipulation of injection brine composition," in EUROPEC/EAGE conference and exhibition, Society of Petroleum Engineers, 2009.

[34] A. Lager, K. J. Webb, C. Black, M. Singleton, and K. S. Sorbie, "Low salinity oil recovery-an experimental investigation," Petrophysics, vol. 49, no. o1, 2008.

[35] F. Mugele, B. Bera, A. Cavalli, I. Siretanu, A. Maestro, M. Duits, M. CohenStuart, D. Van Den Ende, I. Stocker, and I. Collins, "Ion adsorption-induced wetting transition in oil-water-mineral systems," Scientific reports, vol. 5, p. 10519,2015 .

[36] M. E. J. Haagh, I. Sîretanu, M. Duits, and F. Mugele, "Salinity-dependent contact angle alteration in oil/brine/silicate systems: the critical role of divalent cations," Langmuir, vol. 33, no. 14, pp. 3349-3357, 2017.

[37] M. E. Haagh, N. Schilderink, F. Mugele, and M. H. Duits, "Wetting of mineral surfaces by fatty-acid-laden oil and brine: Carbonate effect at elevated temperature," Energy and Fuels, vol. 33, no. 10, pp. 9446-9456, 2019.

[38] J. Gao, S. Nair, M. H. Duits, C. Otto, and F. Mugele, "Combined microfluidicsconfocal Raman microscopy platform for studying enhanced oil recovery mechanisms," Journal of Raman spectroscopy, 2019. 
[39] R. de Ruiter, R. W. Tjerkstra, M. H. Duits, and F. Mugele, "Influence of cationic composition and $\mathrm{pH}$ on the formation of metal stearates at oil-water interfaces," Langmuir, vol. 27, no. 14, pp. 8738-8747, 2011.

[40] A. Ralston and C. Hoerr, "The solubilities of the normal saturated fatty acids," The Journal of Organic Chemistry, vol. 7, no. 6, pp. 546-555, 1942.

[41] W. Beckmann, R. Boistelle, and K. Sato, "Solubility of the a, b, and c polymorphs of stearic acid in decane, methanol, and butanone," Journal of Chemical and Engineering Data, vol. 29, no. 2, pp. 211-214, 1984.

[42] N. J. Everall, "Confocal Raman microscopy: common errors and artefacts," Analyst, vol. 135, no. 10, pp. 2512-2522, 2010.

[43] N. Kumar, L. Wang, I. Siretanu, M. Duits, and F. Mugele, "Salt dependent stability of stearic acid Langmuir-Blodgett films exposed to aqueous electrolytes," Langmuir, vol. 29, no. 17, pp. 5150-5159, 2013.

[44] W. S. Wong, L. Hauer, A. Naga, A. Kaltbeitzel, P. Baumli, R. Berger, M. d'Acunzi, D. Vollmer, and H.-J. Butt, "Adaptive wetting of polydimethylsiloxane," Langmuir, 2020.

[45] G. Sudre, D. Hourdet, C. Creton, F. Cousin, and Y. Tran, "pH-responsive swelling of poly (acrylic acid) brushes synthesized by the grafting onto route," Macromolecular Chemistry and Physics, vol. 214, no. 24, pp. 2882-2890, 2013.

[46] M. P. Weir and A. J. Parnell, "Water soluble responsive polymer brushes,” Polymers, vol. 3, no. 4, pp. 2107-2132, 2011.

[47] W. H. Weber and R. Merlin, Raman scattering in materials science, vol. 42. Springer Science and Business Media, 2013. 
126 CHAPTER 5. IMPROVED 3D-RAMAN IMAGING FOR EOR STUDIES 


\section{Summary}

Monitoring the shift in the energy of photons that are inelastically scattered by molecules forms the basis of Raman spectroscopy - an analytical technique widely used for studying the chemical properties of materials. A Raman spectrum gives us information about the vibrational energies of the molecule that are responsible for this shift, and since this value is unique to a particular vibration, Raman spectroscopy can be used to discriminate different kinds of vibrations in the probed system. However, the phenomenon of Raman scattering is very weak - bombard 100 million $\left(10^{8}\right)$ photons onto a material and only 1 of them would spontaneously Raman scatter. To put this into perspective, one would have a higher probability of flipping a coin 25 times and getting all Heads. Despite this, with better technology, modern day Raman microscopes can acquire a Raman spectrum with appreciable signal-to-noise ratio (SNR) after 1-10 s of accumulation and using a laser dose of $10 \mathrm{~mW} / \mu \mathrm{m}^{2}$.

Naturally, this is not a very long time if only a spectral identification of the probed material is required. In the case where a spatial map of the chemical properties of a material is necessary, especially in heterogeneous materials, Raman imaging has to be performed. Typically, this process involves acquiring multiple spectra by scanning over the substrate in a point-by-point fashion. The total imaging time depends on the resolution of the image and the exposure or accumulation time per pixel. A Raman image made at a resolution of $100 \times 100$ pixels and at an exposure time of 1 
s/pixel would take $\approx_{3}$ hours. This is a considerable amount of time for performing necessary spatial characterization of the material. Other experimental factors which could improve the single-pixel spectral SNR are a higher laser power and/or a higher number of molecules probed, but three questions arise:

- How do we achieve a better spectral SNR if there is a requirement for fast Raman mapping, say at an exposure time of only a few tens of ms/pixel?

- How do we achieve a reliable Raman image of a photo-unstable material, where the allowed laser dosage should be as low as possible to avoid any photoinduced physical and chemical reactions?

- How do we image thin layers of materials or molecules present in low concentrations within the probed volume?

Clearly, the answer to these questions rely on improving the SNR without the modulation of the aforementioned experimental parameters. Processing the acquired Raman hyperspectral dataset (HSD) using mathematical algorithms to achieve efficient denoising is one such solution to improve the SNR. To this end, the mathematical technique of principal component analysis (PCA) was applied to the Raman HSD. This post-processing analysis routine is termed as "algorithm-improved confocal Raman microscopy (ai-CRM)”. The underlying mathematics of the technique as well as a guideline for PCA-based denoising of Raman HSDs is explained in Chapter 2.

In subsequent chapters, the ability of ai-CRM to probe different systems in the low SNR regime is explained. Fast Raman imaging finds its use in the field of $2 \mathrm{D}$ material characterization. However, the ensuing low SNR always hindered the quick and reliable characterization of materials like graphene. For graphene oxide, this problem is exacerbated due to the requirement of a very low laser dosage (in the $\mu \mathrm{W} / \mu \mathrm{m}^{2}$ range) to avoid photo-reduction. The successful application of ai-CRM for fast and non-invasive characterization of $2 \mathrm{D}$ materials is demonstrated in Chapter $\mathbf{3}$, and it is 
shown that the enhancement in SNR enables a scanning rate which is 50 times faster than conventional Raman imaging.

Chapter 4 focuses on the advantage of improved spectral SNR for ultra-sensitive detection and imaging of trace amounts of analytes using graphene enhanced Raman spectroscopy (GERS). Reduced graphene oxide ( $\mathrm{rGO}$ ), fabricated by laser patterning of single graphene oxide (GO) sheets, is demonstrated to be an efficient GERS substrate. The limit of detection of rhodamine-6g (R6G) molecules adsorbed onto rGO and oxidized graphene substrates was determined to be $0.5 \mathrm{nM}$. Additionally, Raman imaging helped in visualizing the distribution of the adsorbed monolayer even at such low bulk concentrations. Lastly, the applications of in-situ ai-CRM to study the adsorption kinetics of R6G and to detect trace adulteration in commercial fruit juice is demonstrated.

In Chapter 5, ai-CRM is utilized to improve the SNR of a large-sized HSD (>50000 spectra), thereby enabling ${ }_{3} \mathrm{D}$ Raman imaging as a feasible characterization tool to study the phenomenon of reactive wettability alteration. As an example, a model system for studying the enhanced oil recovery method of low salinity water flooding was used. In response to a lowering of the ambient brine salinity, picolitre oil droplets atop a surfactant coated mica/silica substrate displayed a change in contact angle, mediated by the redistribution of the surfactant, both of which could be visualized by orthogonal sectioning of the $3 \mathrm{D}$ Raman image. The underlying mechanism for this wettability alteration is also deduced. 


\section{Samenvatting}

Het monitoren van de verschuiving in de energie van fotonen die, inelastisch worden verstrooid door moleculen, vormt de basis van Raman-spectroscopie: een veelgebruikte analytische techniek voor het bestuderen van de chemische eigenschappen van materialen. Een Raman spectrum geeft ons informatie over de vibratieenergieniveaus van het molecuul die verantwoordelijk zijn voor deze verschuiving. Aangezien deze waarde uniek is voor een bepaalde trilling, kan Raman-spectroscopie gebruikt worden om verschillende soorten trillingen in het onderzoek te onderscheiden. Echter, het fenomeen van Raman-verstrooiing is erg zwak - bombardeer 100 miljoen $\left(10^{8}\right)$ fotonen op een materiaal en slechts 1 van hen zou spontaan op die manier verstrooien. Om dit in perspectief te plaatsen bestaat er een grotere kans om 25 keer een munt gooien en steeds kop te krijgen. Desondanks, met betere technologie, kunnen moderne Raman-microscopen een Raman-spectrum produceren met een waarneembare signaal-ruisverhouding (SNR) na 1-10 s meettijd en met een laserdosis van $10 \mathrm{~mW} / \mu \mathrm{m}^{2}$.

Als er enkel een spectrale identificatie moet worden gemaakt voor het onderzochte materiaal, is dit niet erg lang. In het geval dat er een ruimtelijke kaart van de chemische eigenschappen van een materiaal nodig is, vooral in heterogene materialen, moet Raman beeldvorming worden uitgevoerd. In dit geval moeten er meerdere spectra worden verkregen door het substraat puntsgewijs te scannen. De totale 
tijd om een beeld te produceren is afhankelijk van de resolutie van de afbeelding en de meettijd per pixel. Een Raman-afbeelding gemaakt met een resolutie van $100 \mathrm{x}$ 100 pixels en met een meettijd van $1 \mathrm{~s}$ /pixel duurt zo ongeveer 3 uur. Dit vergt dus veel tijd om de ruimtelijke eigenschappen van het materiaal in beeld te brengen. Andere experimentele factoren die de SNR van één spectrum zou kunnen verbeteren zijn een hoger laservermogen en/of een hogere aantal onderzochte moleculen. Er ontstaan drie vragen:

- Hoe bereiken we een betere spectrale SNR als er behoefte is aan een snelle Raman beeldvorming? Bijvoorbeeld bij een meettijd van slechts enkele tientallen ms/pixel?

- Hoe komen we tot een betrouwbaar Raman-beeld van een licht-instabiel materiaal, waarbij de toegestane laserdosering moet zo laag mogelijk is om lichtveroorzaakte fysieke en chemische reacties te voorkomen?

- Hoe brengen we dunne lagen materialen of moleculen, die in lage concentraties aanwezig zijn in het onderzochte volume, in beeld?

Het antwoord op deze vragen is afhankelijk van het verbeteren van de SNR zonder de aanpassing van de bovengenoemde experimentele parameters. Het verwerken van de Raman hyper spectrale dataset (HSD) met behulp van wiskundige algoritmen om efficiënt ruis te onderdrukken is zo'n oplossing om de SNR te verbeteren. Hiertoe werd de wiskundige techniek van hoofdcomponentenanalyse (PCA) toegepast op de Raman HSD. Deze analyse routine wordt algorithm-improved confocal Raman microscopy (ai-CRM)” genoemd. De onderliggende wiskunde van de techniek alsook de richtlijn voor op PCA gebaseerde ruisonderdrukking van Raman HSD's wordt uitgelegd in hoofdstuk 2.

In opeenvolgende hoofdstukken wordt het vermogen van ai-CRM om verschillende systemen in het lage SNR-bereik te onderzoeken uitgelegd. Snelle Ramanbeeldvorming vindt zijn toepassing op het gebied van het karakteriseren van $2 \mathrm{D}$ - 
materiaal. De lage SNR belemmerde altijd de snelle en betrouwbare karakterisering van materialen zoals grafeen. Voor grafeenoxide wordt dit probleem verergerd door de eis van een zeer lage laserdosering (in het $\mu \mathrm{W} / \mu \mathrm{m}^{2}$ bereik) om fotoreductie te voorkomen. De succesvolle toepassing van ai-CRM voor snelle en niet-invasieve karakterisering van $2 \mathrm{D}$-materialen wordt gedemonstreerd in hoofdstuk 3. Het is aangetoond dat de verbetering in SNR een scansnelheid mogelijk maakt die 50 keer sneller is dan conventionele Raman-beeldvorming.

Hoofdstuk 4 richt zich op het voordeel van verbeterde SNR voor ultragevoelige detectie en beeldvorming van hoeveelheden van sporenelementen met behulp van met grafeen versterkt Raman spectroscopie (GERS). Gereduceerd grafeenoxide (rGO), vervaardigd door laser patroneren van één laag grafeenoxide (GO), is een efficiënt GERS substraat gebleken. De detectielimiet van rhodamine-6g (R6G) moleculen, geadsorbeerd aan rGO en geoxideerde grafeensubstraten, bleek o,5 nM te zijn. Aanvullend, Raman-beeldvorming hielp bij het visualiseren van de verdeling van de geadsorbeerde monolaag; zelfs bij zulke lage bulkconcentraties. Ten slotte worden de toepassingen van in-situ ai-CRM gebruikt om de adsorptiekinetiek van R6G te bestuderen en sporenvervalsing in commerciële vruchtensap te detecteren.

In hoofdstuk 5 wordt ai-CRM gebruikt om de SNR van een grote HSD (>50000 spectra) te verbeteren, waardoor $3 \mathrm{D}$ Raman-beeldvorming als een haalbaar meetinstrument kan worden gebruikt om het fenomeen van reactieve verandering van bevochtiging te bestuderen. Als voorbeeld werd er een modelsysteem gebruikt voor het bestuderen van de verbeterde oliewinningsmethode, waarbij water met een laag zoutgehalte wordt geïnjecteerd. Als reactie op een verlaging van het zoutgehalte van de oplossing vertoonden picoliter oliedruppeltjes, op een met oppervlakte-actieve stof gecoat mica / silicasubstraat, een verandering in contacthoek door de herverdeling van de oppervlakte-actieve stof. Beiden zouden kunnen worden gevisualiseerd door een orthogonale doorsnede van het 3D Raman-beeld te maken. Het onderliggende mechanisme voor deze verandering in de bevochtiging wordt ook afgeleid. 


\section{List of publications}

\section{First author}

1. Nair, Sachin, Jun Gao, Qirong Yao, Michael HG Duits, Cees Otto, and Frieder Mugele. "Algorithm-improved high-speed and non-invasive confocal Raman imaging of 2D materials." National Science Review 7, no. 3 (2020): 620-628.

2. Nair, Sachin, Jun Gao, Cees Otto, Michael HG Duits, and Frieder Mugele. "Ultrasensitive Detection and In Situ Imaging of Analytes on Graphene Oxide Analogues Using Enhanced Raman Spectroscopy.” Analytical chemistry 93, no. 38 (2021): 12966-12972.

3. Nair, Sachin, Jun Gao, Cees Otto, Michael HG Duits, and Frieder Mugele. ”Insitu observation of reactive wettability alteration using algorithm-improved confocal Raman microscopy." Journal of Colloid and Interface Science 584 $(2021): 551-560$.

\section{Others}

1. Gao, Jun, Sachin Nair, Michael HG Duits, Cees Otto, and Frieder Mugele. "Combined microfluidics-confocal Raman microscopy platform for studying enhanced oil recovery mechanisms.” Journal of Raman spectroscopy 50, no. 7 (2019): 996-1007. 
2. Ghosh, Udita Uday, Sachin Nair, Anuja Das, Rabibrata Mukherjee, and Sunando DasGupta. "Replicating and resolving wetting and adhesion characteristics of a rose petal." Colloids and Surfaces A: Physicochemical and Engineering Aspects 561 (2019): 9-17.

3. Subramanian, Sri Ganesh, Sachin Nair, and Sunando DasGupta. "Evaporation mediated translation and encapsulation of an aqueous droplet atop a viscoelastic liquid film." Journal of Colloid and Interface Science 581 (2021): 334349. 
"If good things lasted forever, would we appreciate how precious they are?"

Bill Watterson, Calvin and Hobbes

\section{Acknowledgments}

Well hello there! Right to the last few pages I see. I don't blame you, though. What better way to spend some quality time sitting in the audience (or wherever you are) and reading this bit, while I nervously attend to the final questions related to my thesis. The same thesis which summarizes the results of my research in the past 50 months in 140 odd pages, and my gratitude in these few pages. I thank all of you who could make it to my Ph.D. defence ceremony, both physically as well as digitally. It has been a remarkable journey for me, and I have quite a few people to thank for the same.

First of all, I would like to thank my supervisors. Frieder, I thank you for offering me the opportunity to work at PCF. Even though the initial project was focussed more on the EOR aspect, you offered me the flexibility to extend out to other relevant and exciting fields. Your "push" after the second publication also made me more confident in working independently as a researcher. I thank you for the guidance. Cees, I really enjoyed working with you as my co-supervisor. The entire foundation of my thesis relies on your PCA-based denoising technique, and I am glad we could utilize it to study a variety of systems. More importantly, you have always been encouraging - even if it is something small like successfully addressing reviewer comments - your words always raised my morale. I thank you for this. Michael, your guidance as my daily supervisor is deeply appreciated. I really liked the fact that you would be 
genuinely invested in my research progress. Also, the way you would refine my manuscripts and make it more readable and comprehensive made the writing part much easier. I learnt a lot from you. Finally, Jun, you have been the ideal post-doctoral supervisor. You have the ability of predicting potential problems in certain experiments, and you would always guide me in the right direction so that I don't waste a lot of time. Sometimes, I would do those experiments anyway just to know the outcome. Unfortunately for me, you have always been right. :P Thank you for having my back!

I would also like to thank my thesis committee members for taking their time to read my thesis and providing valuable feedback.

Next in line, I would like to thank all my colleagues from PCF for providing me with a comfortable work-environment. Igor, although I never got a chance to work with you directly, I really enjoyed your discussions of new ideas. Dirk, your approach to science and your tendency to understand things at the fundamental level has always been inspiring. Thanks to all my former and recent colleagues: Aditya, Amrutha, Ranabir, Saravana, Ashit, Chamy, Jun, Hao, Cherry (Qierui), Shawshank Su, Arjen, Aram, Simone, Martin, Daniel, Niels, Harmen, Nathalie, Carla, Frank, Isabel, Vincent, Duy, Beybin, Özlem, Stelian, Diana, Amani, Alessandro and Amy. Aditya, Amrutha, Ranabir, Souporni, Simone, Martin, Aram, Denise and Arjen - thank you for all the activities and discussions, the barbecues and the movie nights, the Happy Italy visits, the Friday afternoon "politically correct" discussions and the trips together they were truly the highlights of my life outside the university. Hope we can continue having the infinite house-warming parties. Daniel (also Simone, Ranabir and Aditya), you are the best office mate I could ask for. You always had "important discussions", which were a healthy mental refresh during work. You are also a really talented technician, and I hope you keep staying awesome at your new group. Aram, thank you for always being helpful, irrespective of the time and place. I deeply appreciate it. Beybin (or Nadine?:P), mother of Patik, Princess of Twekkelerveld and 
mentor - thank you for being an awesome and true friend. You have always been supportive and without our discussions and breaks of fresh air, the 4 years of Ph.D. would have felt like an eternity. We started our Ph.D. a month apart, and we end it a week from one another. Congratulations in advance. PS: I can still easily beat you in Asphalt 8 and Street Fighter, obviously without any cheats. :)

I would also like to extend my thanks to my family away from home - Ashrith and Mithun. You guys have always been there for me, since the beginning of the Netherlands chapter of my life. Ash, keep staying awesome and soon I will be seeing you flying one of the skymasters over Enschede. Mithun - engineer, meneer, predictor, and FIFA player of the year - thank you for all the amazing weekends at your place! Good friends are hard to find, and I consider myself extremely lucky to know you two. The rest of the STTM crew - Sam, Dennis, Pavan and Prajwal - thank you for always having me over for any activity/party (not like you guys had any choice:P). Rianne, these past few months have been amazing. Thank you for being my personal translator :P and also helping me with the cover design. I would also like to thank my previous and current housemates - Nina, Sneha, Anmol and Rounak, as well as Ketki, Sibel and Priya. In addition to the nice company, I greatly appreciate the delicious food you guys have always made! Friends back in India (LPPS, Singhania, DMCE, KGP) and abroad (Vegaaaa), I haven't forgotten you. Thank you for being there whenever I felt like talking.

Last but not least, I would like to thank my family. Acha, Amma and Sneha, thank you for your unconditional love and care. Even though I had to leave for the Netherlands on such short notice after my Master's, you were super supportive in letting me pursue my passion for research. I consider myself extremely lucky and I hope this book makes you proud.

Waits for the Beadle to enter... 


\section{About the author}

Sachin Nair was born on the $6^{\text {th }}$ of February, 1993, in Mahayel, Saudi Arabia. He completed his Bachelor's degree in Chemical Engineering at the University of Mumbai, and a Master's degree in Chemical Engineering at the Indian Institute of Technology - Kharagpur campus in India. In 2017, he moved to The Netherlands to pursue his Ph.D. at the Physics of Complex Fluids group within the MESA+ Institute for Nanotechnology and the Department of Science and Technology of the University of Twente. The results of this research are presented in this

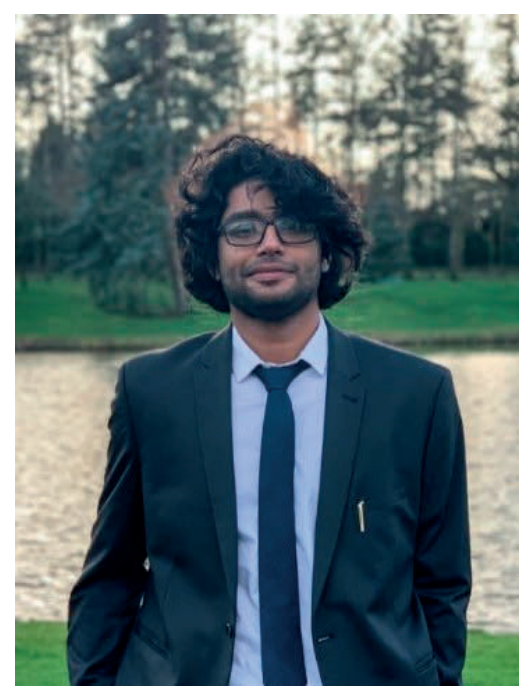
thesis. 


5 